Florida International University FIU Digital Commons

6-17-2010

\title{
Structural Health Monitoring Using Index Based Reasoning For Unmanned Aerial Vehicles
}

Ming Li

Florida International University, mli002@fiu.edu

DOI: $10.25148 /$ etd.FI10080905

Follow this and additional works at: https://digitalcommons.fiu.edu/etd

\section{Recommended Citation}

Li, Ming, "Structural Health Monitoring Using Index Based Reasoning For Unmanned Aerial Vehicles" (2010). FIU Electronic Theses and Dissertations. 241.

https://digitalcommons.fiu.edu/etd/241

This work is brought to you for free and open access by the University Graduate School at FIU Digital Commons. It has been accepted for inclusion in FIU Electronic Theses and Dissertations by an authorized administrator of FIU Digital Commons. For more information, please contact dcc@fiu.edu. 


\title{
FLORIDA INTERNATIONAL UNIVERSITY \\ Miami, Florida
}

\section{STRUCTURAL HEALTH MONITORING USING INDEX BASED REASONING FOR UNMANNED AERIAL VEHICLES}

\author{
A dissertation submitted in partial fulfillment of the \\ requirements for the degree of \\ DOCTOR OF PHILOSOPHY \\ in \\ MECHANICAL ENGINEERING \\ by
}

Ming Li 
To: Dean Amir Mirmiran

College of Engineering and Computing

This dissertation, written by Ming Li, and entitled Structural Health Monitoring using Index Based Reasoning for Unmanned Aerial Vehicles, having been approved in respect to style and intellectual content, is referred to you for judgment.

We have read this dissertation and recommend that it be approved.

Yiding Cao

Sabri Tosunoglu

Shih-Ming Lee

Ibrahim Nur Tansel, Major Professor

Date of Defense: June 17, 2010

The dissertation of Ming Li is approved.

\begin{tabular}{r} 
Dean Amir Mirmiran \\
College of Engineering and Computing \\
\hline Interim Dean Kevin O'Shea \\
University Graduate School
\end{tabular}

Florida International University, 2010 


\section{ACKNOWLEDGMENTS}

First of all, I would like to give my deepest thanks to my major professor, Dr. Ibrahim Nur Tansel, for his great tolerance, patience, and understanding. This work would not be finished without his guidance, support and encouragement. Also, I wish to give a big thanks to the members of my committee, Dr. Yiding Cao, Dr. Sabri Tosunoglu and Dr. Shih-Ming Lee, for their willingness, guidance and help with my work.

I would like to extend special thanks to Dr. Cesar Levy and Dr. Weiyu Bao, for their endless help and support.

I also would like to thank my colleagues and friends, Dr. Xiaohua Li, Dr. Ming Feng, Dr. Ju Sun, Kimberly L. Bickraj, Dr. Aylin Yenilmez, Dr. Ahmet Yapici, Dr. Mustafa Demetegul, Carlos Andres, Srikanth Korla, Gurjiwan Singh, Gurjiashan Singh, Jimmy Sandoval, Esteban Cifuentes, and Alberto Garcia, etc. Their support and cooperation made this work possible.

Last but not the least, I wish to thank the University Graduate School of Florida International University for providing me with the Initiate Teaching Strategy Scholarship and the Dissertation Year Fellowship, so that I had this great opportunity to pursue a graduate degree. 
ABSTRACT OF THE DISSERTATION

STRUCTRUAL HEALTH MONITORING USING INDEX BASED REASONING FOR UNMANNED AERIAL VEHICLES

by

Ming Li

Florida International University, 2010

Miami, Florida

Professor Ibrahim Nur Tansel, Major Professor

Unmanned Aerial Vehicles (UAVs) may develop cracks, erosion, delamination or other damages due to aging, fatigue or extreme loads. Identifying these damages is critical for the safe and reliable operation of the systems.

Structural Health Monitoring (SHM) is capable of determining the conditions of systems automatically and continually through processing and interpreting the data collected from a network of sensors embedded into the systems. With the desired awareness of the systems' health conditions, SHM can greatly reduce operational cost and speed up maintenance processes.

The purpose of this study is to develop an effective, low-cost, flexible and fault tolerant structural health monitoring system. The proposed Index Based Reasoning (IBR) system started as a simple look-up-table based diagnostic system. Later, Fast Fourier Transformation analysis and neural network diagnosis with self-learning capabilities were added. The current version is capable of classifying different health conditions with the learned characteristic patterns, after training with the sensory data acquired from the operating system under different status. 
The proposed IBR systems are hierarchy and distributed networks deployed into systems to monitor their health conditions. Each IBR node processes the sensory data to extract the features of the signal. Classifying tools are then used to evaluate the local conditions with health index (HI) values. The HI values will be carried to other IBR nodes in the next level of the structured network. The overall health condition of the system can be obtained by evaluating all the local health conditions.

The performance of IBR systems has been evaluated by both simulation and experimental studies. The IBR system has been proven successful on simulated cases of a turbojet engine, a high displacement actuator, and a quad rotor helicopter. For its application on experimental data of a four rotor helicopter, IBR also performed acceptably accurate. The proposed IBR system is a perfect fit for the low-cost UAVs to be the onboard structural health management system. It can also be a backup system for aircraft and advanced Space Utility Vehicles. 


\section{TABLE OF CONTENTS}

CHAPTER

PAGE

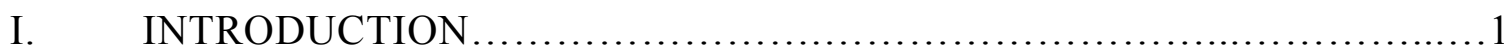

1.1 Structural Health Monitoring.............................................

1.2 Objective of Present Study ................................................

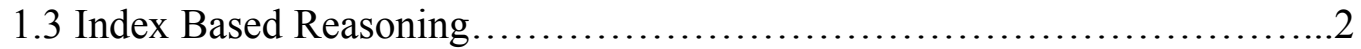

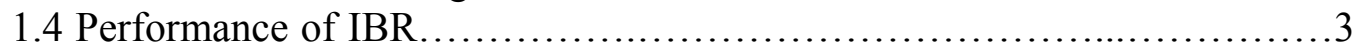

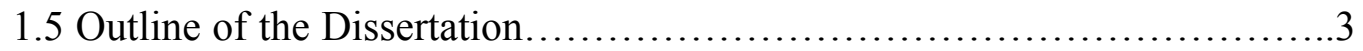

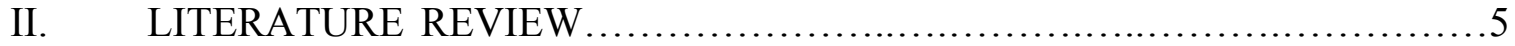

2.1 Structural Health Monitoring..........................................

2.2 Research Focuses..................................................... 7

2.3 Data Processing of SHM.......................................... 8

2.3.1 Feature Extracting.......................................... 10

2.3.1.1 Vibration Based Condition Monitoring.....................10

2.3.1.2 Lamb Wave Based Health Monitoring.......................12

2.3.1.3 Feature Extracting Methods............................14

2.3.2 Decision Making.............................................. 17

III. THEORETICAL BACKGROUND OF DATA PROCESSING ....................21

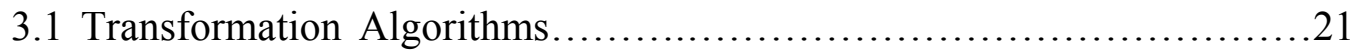

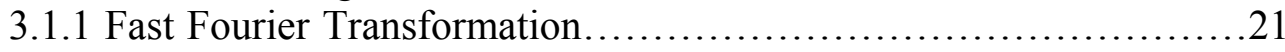

3.1.2 Short Time Fourier Transformation.............................22

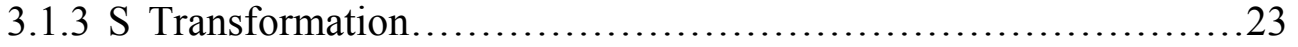

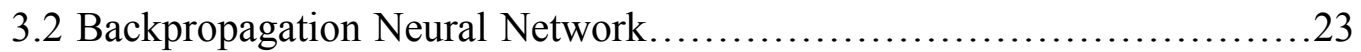

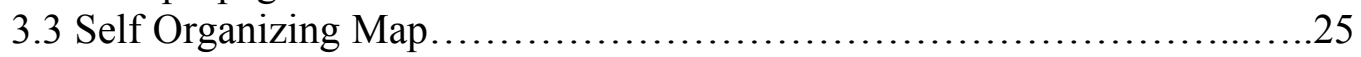

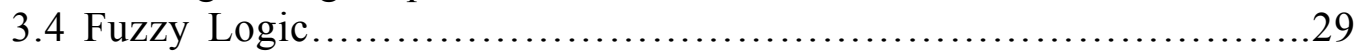

3.4.1 Mamdani and Sugeno Methods................................... 31

3.4.2 Neuro-Fuzzy System........................................... 32

3.5 Dynamic Model of a Four Rotor Helicopter..............................32

IV. INTRODUCTION TO INDEX BASED REASONING .......................36

4.1 Index Based Reasoning.............................................

4.2 Graphic User Interface Programmer for IBR .......................... 41

4.3 Single Chip Index Based Reasoner.......................................45

V. PERFORMANCE OF IBR ON SIMULATED SYSTEMS...................54

5.1 Simulation of a High Displacement Actuator...............................54

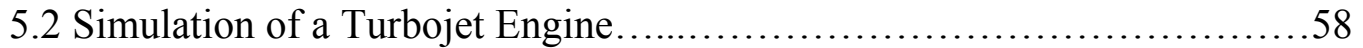

5.3 Simulation of a Four Rotor Helicopter................................62

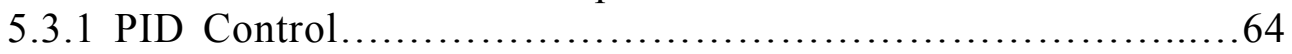

5.3.2 Fuzzy Logic Control...............................................69

5.4 Simulation on FEM Estimation of Carbon Fiber Tubes......................73 
5.5 Conclusions .................................................... 79

VI. PERFORMANCE OF IBR ON EXPERIMENTAL DATA......................81

6.1 Experiments on Composite Tubes..............................81

6.1.1 Vibration Based Monitoring......................... 82

6.1.1.1 Hole Damage...................................8 82

6.1.1.2 Slot Damage......................................92

6.1.2 Sweep Sine Based Monitoring..................................... 98

6.1.3 Lamb Wave Based Monitoring................................110

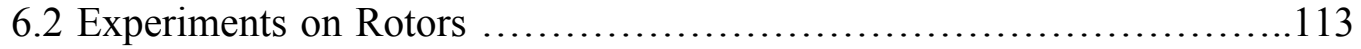

6.3 Conclusions.................................................... 118

VII. CONCLUSIONS AND FUTURE WORK................................... 120

7.1 Conclusions.................................................... 120

7.2 Future Work................................................... 123

LIST OF REFERENCES..................................................... 125

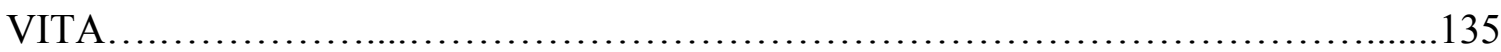




\section{LIST OF TABLES}

TABLE

PAGE

1. Memory Usage of Different Applications (Variables Declared as Integer)...........51

2. Memory Usage of Different Applications (Variables Declared as Byte)..............51

3. Ansys FEM Simulation Dataset............................................... 75

4. Performance of the Linear Model............................................... 78

5. Frequency Responses with Respect to Size and Location of Holes.......................84

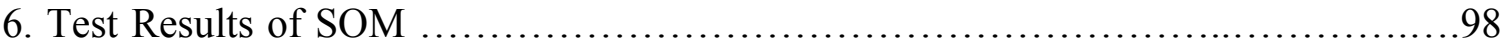




\section{LIST OF FIGURES}

FIGURE

PAGE

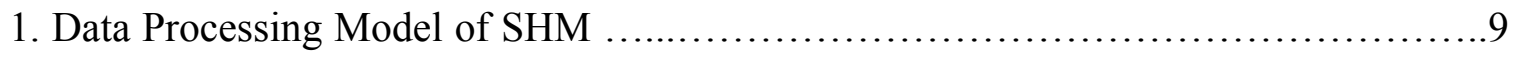

2. Structure of a Backpropagation Neural Network..................................24

3. Structure of a Self Organizing Map.............................................27

4. Process of a Fuzzy Logic System............................................29

5. Schematic Diagram of a Four Rotor Helicopter................................33

6. Example of the IBR System............................................. 37

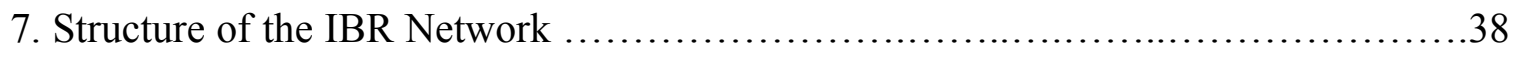

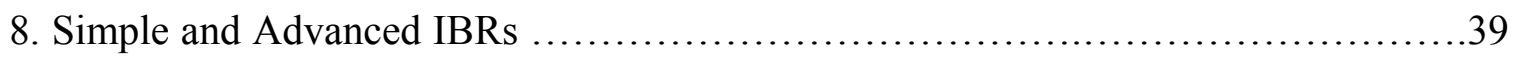

9. Graphic User Interface of the 3D IBR Programmer..............................4

10. Programming the IBR Node

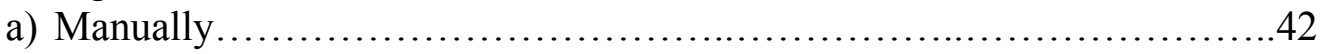

b) Automatically.................................................. 42

11. Neural Network Training Results

a) Original Pattern...................................................43

b) After 10,000 Iterations (Err 5.17\%)............................... 43

c) After 30,000 Iterations (Err 4.17\%).............................. 44

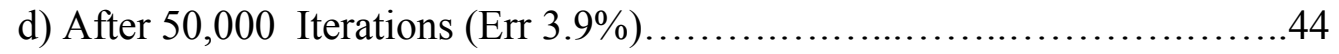

12. GUI Example of the IBR Structure

a) Time Domain Input................................................ 45

b) FFT Analysis Input...................................................

13. Information Exchange between the SCIBR Environment and

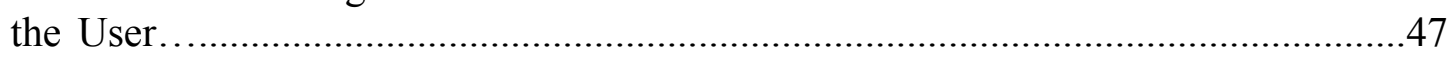

14. Training Data Input Form............................................. 48

15. Boundaries of Signals and Choices of Classifications...........................49

16. Prepared MikroBasic Code Based on Selected Classifications....................49 
17. Editor of the MicroBasic Integrated Compiler....................................50

18. Boundaries of Signals from Four Sensors.....................................52

19. Compilation Results in MikroBasic.............................................52

20. Diagram of a Simulated HDA and its Health Monitoring System...................55

21. Simulink Model of the Piezomotor and its ARMAX Based IBR System..............57

22. Variation of HI Values During the Transition Period

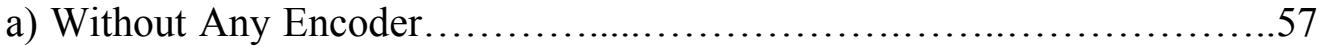

b) With ARMAX Based Encoder..........................................5

23. Diagram of a Simplified Engine with Support Structures and

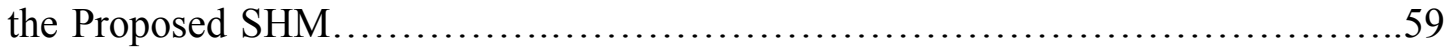

24. Simulink Model of the Engine and the Proposed Health Monitoring System.

25. Diagrams of IBR Nodes for the Simulated Engine

a) Block diagram of IBR1 and IBR3 ..................................61

b) Block diagram of IBR2 and IBR4 ..................................61

26. HI Values from Warming Up to Bearing Failure...................................62

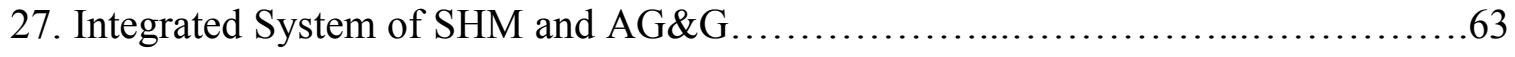

28. Simulink Model of the Four Rotor Helicopter System

a) Four Rotor Helicopter and its Health Monitoring System...................65

b) Details of the Health Monitoring System ................................66

29. Trajectory of the Simulated Four Rotor Helicopter

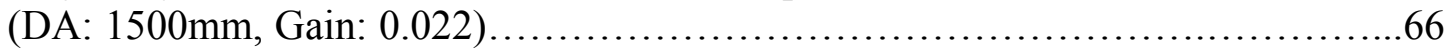

30. Trajectories with Different Gains (DA: $6000 \mathrm{~mm}$ )

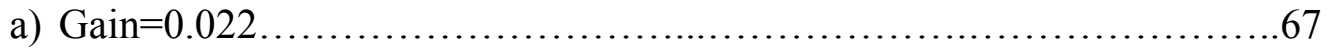

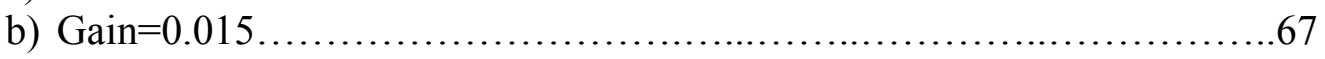

31. Trajectories under Different Conditions........................................68

32. Simulink Model of Fuzzy Logic Control..........................................69

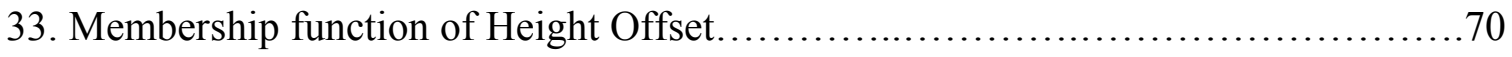


34. Membership Function of Vertical Speed..........................................71

35. Membership Function of Output Force ......................................... 71

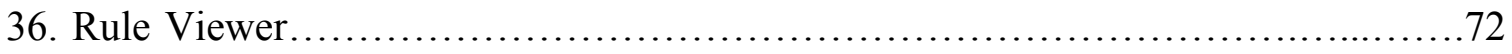

37. Simulink Result under Perfect Condition.......................................72

38. Simulink Result When One Rotor Failed at 8s...................................73

39. FEM Model of the Composite Tube in Ansys......................................74

40. Fitness of Linear, Exponential, Logarithmic and Power Law Models Matching the Trend of the Simulation Data ..........................................76

41. Fitting and Mapping of the Exponential Model......................................77

42. Comparison of the Accuracy of Four Different Models..................................78

43. Dimensions of the Carbon Fiber Tube ........................................... 82

44. Dimensions of the Tubes with Defects (Left), and the Tubes (Right)......................83

45. Frequency Responses of Tubes with a Hole of Different Size and

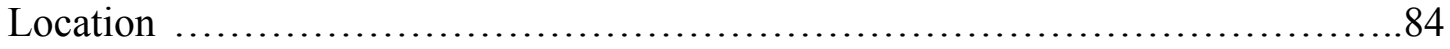

46. Experimental Setup to Monitor the Vibration of Composite Tubes.....................85

47. Frequency Responses of a Perfect Tube (Laser Vibrometer and

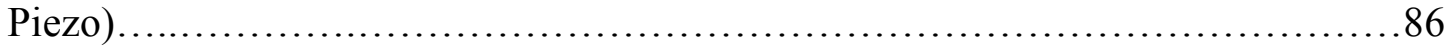

48. Sweep Sine Measurement of a Perfect Tube (Laser Vibrometer and

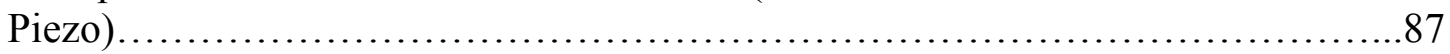

49. Frequency Responses of Perfect and Defect Tubes (Laser Vibrometer)..............88

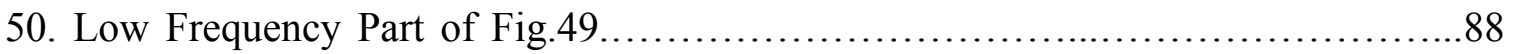

51. Frequency Responses of a Perfect Tube When the Rotor Was Running...............89

52. Experimental Setup (Rotor of One Arm Was Running at Fixed RPM)................90

53. Experimental Setup to Identify Damages of One Arm (Helicopter Was Running). 
54. Dynamic Responses of Perfect and Defect Tubes When the Rotor Was Running at Low RPM and High RPM....................................92

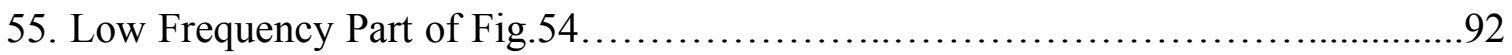

56. Experimental Setup (Vibration Test System).....................................93

57. Perfect Tube and Damaged Tubes with the Slot ..................................94

58. Simulink Model of the Proposed IBR+SOM Approach............................94

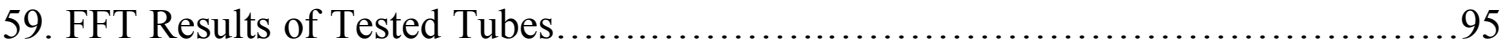

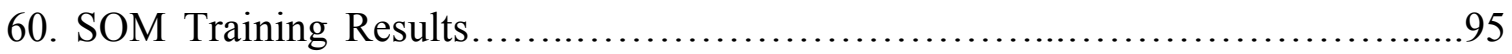

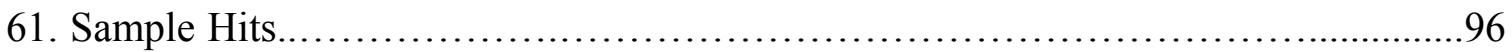

62. Clusters Labeled with Different Types..............................................97

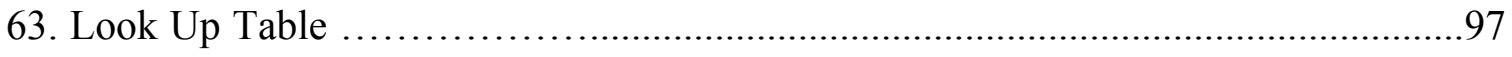

64. Proposed Procedure Using SOM-IBR Combination for Classification.................99

65. Perfect and Damaged Tubes Bonded with Piezoelectric

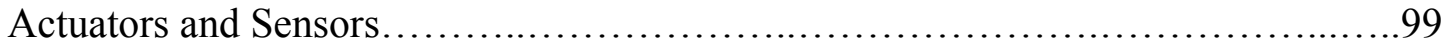

66. Sweep Sine Responses of Perfect and Damaged Tubes...............................100

67. Visualization of the Neighbor Weight Distances after the Training...................101

68. Weight Positions of the SOM......................................................................102

69. Four Clusters Corresponding to Different Structural Integrity Levels...............103

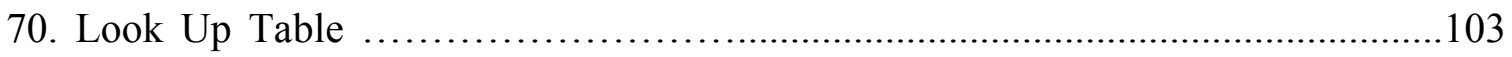

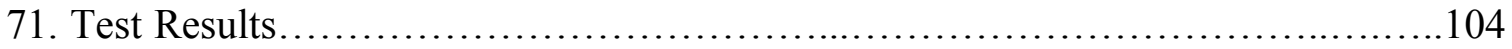

72. Experimental Setup for Sweep Sine Based Monitoring..............................................105

73. S Transform of the Sensory Signals

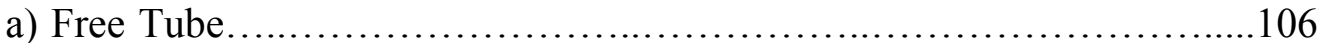

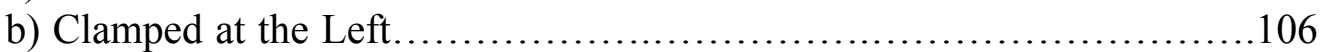

c) Clamped at the Middle................................................

d) Clamped at the Right............................................... 106 
74. Contour of Peaks

75. Maximum Amplitude Along Time Axis

76. Maximum Amplitude Along Frequency Axis............................................... 108

77. Backpropagation Neural Network Model .................................................. 109

78. Perfect Tube and Defect Tube with a Notch................................................110

79. Experimental Setup for Lamb Wave Based Monitoring.........................111

80. S Transform of Dynamic Responses of Perfect and Defect Tubes

a) Perfect Tube.

b) Perfect Tube Clamped at the Left....................................111

c) Perfect Tube Clamped at the Middle.....................................112

d) Perfect Tube Clamped at the Right..................................112

e) Defect Tube .................................................... 112

81. Proposed Procedure for the Inspection of the Propellers .....................................114

82. Perfect and Damaged Propellers ............................................. 114

83. Experimental Setup to Measure the Frequency Responses of the Rotors...........114

84. Frequency Responses of the Arms Measured by a Dynamometer..........................115

85. Frequency Characteristics of the Arms Measured by a Piezoelectric Strip...........115

86. IBR Classification Model Based on the Ratio of the First Two Peaks...............116

87. Frequency Responses of Different Combination of Tubes and Propellers

a) Perfect Tube with Different Propellers

b) Perfect Propeller with Different Tubes..... 


\section{CHAPTER I}

\section{INTRUDUCTION}

\subsection{Structural Health Monitoring}

Structures of Unmanned Aerial Vehicles (UAVs) may develop cracks, erosion, delamination or other damages due to aging, fatigue or extreme loads. Identifying these damages is critical for the safe and reliable operation of the systems. In the past, various tedious inspection processes and schedule based maintenances had to be applied to keep these systems operational. The Non-Destructive Evaluation (NDE) takes very long time and only the critical locations could be evaluated.

Structural Health Monitoring (SHM) has been proposed to evaluate the integrity of systems in order to lower the operational cost and speed up the maintenance process. SHM determines the conditions of the systems automatically and continually through processing and interpreting the data collected from a network of sensors embedded into the systems. Thus, structural damages can be identified effectively in an early stage so that the rest of the mission can be either completed by modifying the control strategy or aborted to avoid total loss of the system. SHM is not only the fundamental requirement for developing self-diagnosing structures, it also has the potential for developing selfhealing and more sustainable systems.

\subsection{Objective of Present Study}

The purpose of this study is to develop an efficient, low-cost, flexible and faulttolerant health monitoring system. The Index Based Reasoning (IBR) system was developed for this purpose. The capability of IBR systems was improved step by step. When IBR was first introduced, it was a simple look-up-table (LUT) based diagnostic 
system. It had a simple graphic user interface which enabled users to customize the desired LUT for identification. Then, vibration based, sweep sine based, and Lamb wave based monitoring analysis were added to enhance the performance of IBR. Dynamic frequency responses were collected by piezoelectric elements and other sensors, and Fast Fourier Transformation (FFT) and S transformation were applied to extract the characteristics of the frequency response. Neural networks with self-learning capabilities were utilized for diagnostic reasoning to identify perfect and defect structural elements. After training with the characteristic patterns of the sensory data, the IBR is capable of identifying the different health conditions under which the system is operating.

\subsection{Index Based Reasoning}

The proposed IBR system is a hierarchy and distributed network deployed into the system to monitor the health of the system. Depending on the complexity of the system, there may be just one or thousands of IBR nodes. Each node processes the sensory data with FFT or other methods to extract the features of the signal. Classifying tools, such as LUTs, neural networks, fuzzy logic or genetic algorithms, will be used to evaluate the local health condition with an index value. If the dynamic characteristics of the input signal are more important and complicated, the advanced IBR nodes may have more than one encoded input. The Index History Evaluator (IHE) will determine the HI value with respect to the history of the HI values, so that the accuracy of the estimations can be improved in spite of noise. The HI values will be carried to another IBR node in the next level of the structured network. The overall health condition of the system can be obtained by evaluating all the local health conditions. Then, the optimized control strategy can be determined to operate the system effectively and reliably. 


\subsection{Performance of IBR}

In this study, the performance of IBR has been proved by simulation and experimental works. For the simulated models of a High Distance Actuator (HDA) and a turbojet engine, SR-30, the IBR systems accurately identified the different health conditions of the systems and modified the control strategy in accordance with the structural integrity. A small four rotor helicopter was also simulated; it's Autonomous Guidance and Control (AG\&C) system was integrated with IBR based SHM. The simulated control systems, the PID and the fuzzy logic systems, successfully identified the simulated health conditions and guided the four rotor helicopter to the target under these conditions.

The IBR system was also been testified by the experimental work on the components of the four rotor helicopter: the carbon fiber tubes and the propellers. Some of these structural components were damaged on purpose to generate training data for the IBR. Piezoelectric actuators and sensors were bonded on the structures to collect dynamic information. After the training, IBR was able to identify the occurrence of the problem and estimate the severity and location of the damage. The accuracy of the evaluation was acceptable.

\subsection{Outline of the Dissertation}

Chapter II provides detailed literature reviews on SHM, vibration based health monitoring and Lamb wave based health monitoring. Theoretical background of the methods used for feature extracting and diagnostic reasoning in SHM is presented in Chapter III. Chapter IV introduces the IBR system, including the structure of IBR, the Graphic User Interface programer to train IBR nodes and generate MicroBasic code. 
Chapter V illustrates the IBR models and their performance on simulated systems, a turbojet engine, a HDA and a quad rotor helicopter. In Chapter VI, the applications of IBR on experimental data are shown. Experimental investigations on the structural elements of a four rotor helicopter are given. The frequency responses of the damaged and the undamaged carbon fiber frame members and propellers were collected by various sensors. The analysis and evaluation of IBR on these sensory data are also presented in this Chapter. Conclusions and recommendations for future studies are briefed in Chapter VII. 


\section{CHAPTER II \\ LITERATURE REVIEW}

\subsection{Structural Health Monitoring}

In the past, to evaluate the structural integrity of aerospace, mechanical and civil

engineering infrastructure, tedious inspection processes and schedule-based

maintenances had to be carried out manually. Many sections of the vehicles had to be disassembled. These passive investigations were usually time and labor-intensive. They were also not objective, because the evaluation results mainly depended on the judgment of operators. In addition, these inspection and maintenance processes were not cost effective, since some components of the system had to be replaced at fixed time intervals even though they still could operate profitably (Zhang et al. 2005).

In the 50s and 60s, Non-Destructive Evaluation technologies started to be applied to investigate the structural integrity of complex systems. The evaluation processes had been significantly enhanced by NDE methods. NDE refers to a wide range of measurement technologies and analysis methodologies that evaluate the properties of a material, component or system without causing any damage to the structures being inspected. NDE techniques such as ultra sonic scanning, X-rays inspection, thermographic imaging, holography mapping, eddy current probes, acoustic emissions, etc., are widely utilized in scientific and industrial applications (Bayraktara et al. 2008).

However, these technologies often require specialized non-destructive testing equipments and procedures, which are labor and equipment-intensive. Most of these non-destructive 
evaluation methodologies are impractical to be implemented onboard, and cannot operate automatically for in situ monitoring (Yen and Meesad 2001). Moreover, many NDE methods only can be applied to identify surface damages; even though some methods can reveal inner problems, they lack accuracy in providing quantitative information (Bayraktara et al. 2008).

With the development of modern engineering technologies, aerospace structures become more and more complicated, and needed more sophisticated test methods. At the same time, a downsized workforce, a declining budget and a shorter interim operating period have further complicated the task of maintenance and sustainment (Yen and Meesad 2001). To solve this problem, SHM has been developed during the past several decades. SHM technologies applied ground-based or embedded onboard will significantly improve the reliability and availability of complex systems, reduce the amount of time and manpower required to prepare and maintain the systems, and reduce the number of system failures and life casualties induced by damages to structures.

The term SHM is used to describe a complete system of technologies and methodologies that can provide the capability to monitor structural integrity and identify the existence, severity and location of damages (Dlmasry and Johnson 2004). By integrating contextual and timely data, information, and knowledge (DIaK), SHM can achieve capabilities of determining the system's structural integrity in aggregate performance: identifying the existence of damages, detecting severity and location of 
damages, predicting future anomalies and optimizing the corresponding system behavior

(Figueroa et al. 2006). With the desired awareness of the system's health conditions,

SHM can fully exploit the life time of the system to achieve the maximum cost benefit.

SHM is total health management. The concept of SHM starts from the design phase and carries through all the life cycle of the system.

\subsection{Research Focuses}

Tremendous progress has been achieved in SHM over the last two decades.

These achievements can be attributed to the rapid development of multidisciplinary tools and techniques of mechanics, materials science, electrical and computer engineering, etc. Generally speaking, the research of SHM focuses on the following fields:

- Materials

Researches have been carried out to develop smart materials for structures. Piezoelectrics, electrostrictives, magnetostrictives, shape memory alloys, biomimetic and conductive polymers, electrochromic coatings, magnetorheological, electrorheological fluids, etc. are samples that have already been available for adaptive structures (Garg et al. 2001). 
Many kinds of smart materials are either embedded with sensor networks or sensors themselves. Since they can sense the changes of the ambient environment, smart materials will find a variety of applications in developing built-in self-diagnosis, self-healing and more sustainable structures.

- Sensors

* New Sensors

With the recent advances in sensing technologies, it has become increasingly common to permanently attach sensors to structures in order to monitor a variety of parameters. These sensors can facilitate the continuous monitoring of the structure. The measurements taken by sensors can give an indication of the current condition and estimate the remaining life of the structure. Thus, it can keep the maintenance downtime of the structure to a minimum (Konstantinidis et al. 2007).

* Sensor Placement 
The type and number of sensors applied in SHM, and their locations in the system are critical issues. According to the concept of total health management, the design of sensor network should be accompanied with the design of the system. The fault propagation in the system should also be investigated to design the placement of sensors.

* Sensor Validation

As more and more sensors are embedded to detect the damage of the system, the damage of the sensors themselves becomes an issue. Identifying damages to the sensor network and improving its fault tolerant ability have attracted more and more interest.

- Data Processing

Data Processing is one of the most crucial issues in SHM. After setting up the sensor network, different kinds of algorithms and technologies chosen to process the data and make the decision may determine the effectiveness and accuracy of SHM. 


\subsection{Data Processing of SHM}

In this research, we mainly focused on the data processing issue. Since the application of SHM varies widely across different disciplines, there is no "onesize-fits-all" approach to build the health monitoring capabilities for a system (Patterson-Hine et al. 2005). Fig.1 shows the model used in this study to identify the structural integrity of the system (Worden and Dulieu-Barton 2004; Carden and Fanning 2004; Zou et al. 2000).

Sensing $\Rightarrow \begin{gathered}\text { Signal } \\ \text { Processing }\end{gathered} \Rightarrow \begin{gathered}\text { Feature } \\ \text { Extracting }\end{gathered} \Rightarrow \begin{gathered}\text { Pattern } \\ \text { Processing }\end{gathered} \Rightarrow \begin{gathered}\text { Condition } \\ \text { Evaluating }\end{gathered} \Rightarrow \begin{aligned} & \text { Decision } \\ & \text { Making }\end{aligned}$

Fig.1 Data Processing Model of SHM

From the diagram, the information of the ambient environment measured by sensors deploying all around the system will be pre-processed to make it ready for the next step. Analog and digital signal conditioners or filters can be applied to amplify the signal and eliminate the noise. Then, the features indicating the change of systems will be extracted. With the assumption that damages will change the 
characteristics of the system, damage identification is a statistical pattern recognition problem (Carden and Fanning 2004). The property patterns of the structural components and systems will be compared with the controlled undamaged structures and systems to identify the existence of damages and evaluate the location and severity of the damages. Thus, the conditions of the system can be estimated automatically and continually through the life cycle of the system. The structural damage can be identified effectively in an early stage, so that control strategy can be modified to prolong the service life of the system, or condition based down time maintenance will be carried out to avoid total loss of the system.

The diagram in Fig.1 briefly demonstrates two main objectives of SHM applications:

- To characterize the property which can indicate the change of the structural integrity of the system 
- To determine the health condition of the system and optimize the control strategy for the rest of the mission

\subsubsection{Feature Extracting}

\subsubsection{Vibration Based Condition Monitoring}

The objective of the first step is to extract features that can distinguish the conditions of the system. Vibration based condition monitoring can be applied to achieve this goal (Fritzen 2005). Vibration based condition monitoring refers to the use of non-destructive methods to acquire sensory signals of the operating system and analyze the system characteristics in time, frequency or modal domain, in order to identify dynamic response changes which may indicate damages or anomalies (Carden and Fanning 2004). This approach extracts modal properties of the system from vibration information to solve pattern recognition problems in order to identify between undamaged and damaged components (Zou et al. 2000). 
In vibration based condition monitoring, piezoelectric sensors are commonly utilized as sensors and actuators because of their characteristics of changing length with the application of an electric field, and vice versa. Other than piezos, accelerometers and strain gages are samples of commercially available sensors that have been widely applied in system condition monitoring.

According to different applications, the characteristics that can be extracted to identify the change of structures and systems may be different. Banks et al. (1996) proved that changes to physical parameters, such as damping and stiffness, could be identified to detect the damage and locate the problem area. Cawley and Ray (1998) successfully demonstrated that the frequency changes indicated the damage to the system. Cawley and Adams (1979) showed that the ratio of frequency changes in different modes was a function of the damage location. Sofge (1994) identified the first four vibrational modes to evaluate the structural delamination and degradation of a cantilever composite beam.

Although system state measurements are always made in time domain, operating information acquired through the sensors can be transformed into frequency domain, 
where distinct signatures of the sensory data can be observed. Further analysis of the frequency information will lead to extraction of modal domain features. However, there may be information loss during the conversion between time domain and other domains. Commonly, instinctive features observed in one or in combination of different domains can be utilized to demonstrate the change of the system's integrity.

Natural frequency is the fundamental property of the system, which depends on the mass and stiffness of the structure. Because changes of structures are often accompanied by losses of mass or stiffness, the natural frequency shift is a main issue in estimating the condition of the system. Other than that, to measure the natural frequency of the system, only one or a few sensors are needed to acquire the vibration signals. However, it needs multiple sensors to extract other modal features. Sometimes, the complete and accurate modal parameters cannot be obtained, even with a large number of sensors. Due to these concerns, a lot of researches detect the structural damage based on changes of the natural frequency. Dilena and Morassi (2009) illustrated that the natural frequency shifts of axially vibrating rods indicated stiffness variations induced by structural degradation. White et al. (2007) investigated the frequency response of 
adhesively bonded joints to detect debondings in scarf and overply repairs. Salawu (1997) reviewed 65 papers utilizing natural frequency to monitor the integrity change of the system. However, the effectiveness of monitoring the system condition change based on natural frequencies is case dependent. Materials, structures, supporting conditions and other ambient environmental parameters all can affect the shifts of the natural frequency.

To date, researchers still cannot reach the agreement on the suitability of evaluating the system condition based on the modal domain parameters (Carden and Fanning 2004). One school of researchers argues that since damages are local events while modal data indicates global characteristics, they are not sufficiently sensitive to the integrity change of the system. However, others disagree with this opinion. Both sides have experimental research carried out to support their arguments, but since only specific structures have been tested, none have been proven in a fundamental sense (Alampalli et al. 1997). In spite of the argument, the majority of the research on vibration based condition monitoring utilizes extracted modal features as the main reference of system states. Natural frequencies and mode 
shapes are commonly obtained and interpreted because of the easiness of implementations. According to the diversities of damages, structures and systems, approaches based on the information of only one domain are not enough to solve the health monitoring problems. A broad range of applications investigate characteristic features of all the domains to determine the health conditions of the system.

\subsubsection{Lamb Wave Based Health Monitoring}

Lamb wave based health monitoring is another efficient method for SHM (Lu

et al. 2008). Lamb waves were first introduced by English mathematician, Horace Lamb, in 1917. Lamb waves are two dimensional acoustic waves propagating in a solid material, bounded by parallel planes. Lamb waves are divided into two infinite sets of modes: the symmetric $\left(S_{n}\right)$ and antisymmetric $\left(A_{n}\right)$ modes (Lamb 1917). The propagation properties of Lamb waves depend on the excitation frequency, the thickness, and material properties of the structures (Pei et al. 
1995). Since, in reality, Lamb waves are propagated in the finite boundary of structures, they are also called "Guided Lamb Waves (Luangvilai et al. 2002)."

Lamb waves are sensitive to the interference of propagation between the actuators and sensors. The propagation variations of Lamb waves due to the changes in thickness and media properties can be employed to detect damages not only on the surface, but also in between of the structures. Since Lamb waves can propagate along considerable distances without much attenuation, they can be applied to inspect a broad area quickly with less transducers (Su et al. 2006). Guided Lamb waves have been widely utilized to assess the integrity of plates and cylindrical tubes during the last two decades. Degertekin excited Lamb waves ranged from 200 to $500 \mathrm{kHz}$ through point-like Hertzian contacts using a PZT piezoelectric transducer and quartz buffer rod. In their study, the single mode of Lamb wave, the antisymmetric mode, was generated; its zero order wave was utilized to identify the delamination in composite materials (Degertekin and Khuri-Yakub 1997). Kessler et al. (2001) optimized the geometry of 
piezoelectric transducers and the driving parameters to excite the Lamb waves, and identified delamination in narrow composite coupons, sandwich beams, and built-up structures through wavelet decomposition of transmitted signals. Diamanti et al. (2004) employed a linear array of piezoceramic patches to transmit and receive the Lamb wave in a large multidirectional composite board; the position and the severity of impact damages were successfully detected.

Lamb wave based condition monitoring is cost-effective and reliable. However, the dispersive characteristics and the existence of multiple wave modes make it extremely complicated to interpret Lamb wave signals. The mode and the frequency of excited Lamb waves should be determined carefully for specific damage detecting applications (Rose 2000). In addition, the reflection from boundaries of the structure may interfere with signals caused by damages, which make it even more complicated to isolate useful information indicating condition variations (Su et al. 2006). Although substantial research has explored Lamb wave based damage identification techniques, and valuable developments 
have been achieved; most of these studies were carried out in a laboratory, and practical methods via Lamb wave testing have yet to be developed for real-time health monitoring applications (Diamanti et al. 2004).

\subsubsection{Feature Extracting Methods}

Statistic analysis can be used to extract features. Mean, standard deviation, skewness, kurtosis, etc. are commonly calculated to extract useful information from signals. However, to assess the dynamic operating signals, FFT, wavelet and other transformation methods are used more frequently to identify prominent features in frequency or modal domain.

FFT is an efficient method to transform sensory signals from time domain to frequency domain, where dominant frequency characteristics of the signal can be easily extracted and analyzed. FFT has long been applied to analyze signals for SHM (Carden and Fanning 2004). Loh and Lee (1997) utilized FFT spectral analysis of seismic responses to characterize the structural dynamics of a 5-span continuous box-girder bridge. Loewke et al. (2005) investigated the possibility of implementing two dimensional FFT to decompose signals from micro sensors embedded into composite materials, and found this method was extremely efficient to assess the health condition if it was indicated by the low frequency component.

However, when signals are transferred to the frequency-domain, the information of time will be lost. In many applications of SHM, the time-frequency representation (TFR) of sensory data is desirable. The short time Fourier transformation (STFT) (Hurlebaus et 
al. 2001), wavelet transformation (WT) (Legendre et al. 2000) and S transformation

(ST) (Wang and Tansel 2007) may be used to study the time-varying characteristics of the spectral information.

The STFT had been proven sensitive enough to detect delamination in fiberreinforced composites and steel-reinforced concrete. The peak amplitude of STFT was extracted and compared between the damaged and the undamaged samples to estimate the variation of structure integrity (Wang et al. 2001). Kaphle et al. (2009) used STFT to evaluate waveform data collected by acoustic emission sensors attached to a slab-ongirder bridge, and their results proved that STFT was superior to traditional frequency analysis in extracting useful information.

Since a constant time window is utilized to calculate STFT, the resolution of both time and frequency are limited. A wide window gives relatively better frequency resolution, while a narrow window gives better time resolution (Niethammer et al. 2001).

To solve this problem, WT, ST and other Fourier derived multi-resolution methods have been developed to adapt variable window sizes in accordance with the desirable frequency information. If low-frequency representation is needed, the longer window will be applied; when high frequency information is desired, the shorter window will be used (Kaphle et al. 2009).

Wavelet transformation has the ability to provide good time and frequency resolution for the joint time-frequency representation of dynamic signals. Thus, as an efficient transformation algorithm, WT has been widely utilized for signal processing in SHM 
(Reda Taha et al. 2006). Smith et al. (2007) investigated the per-level thresholding (PLT) method to suppress noise and extract characteristic features using Haar, Daubechies, and Morlet wavelets, and verified its feasibility using the real flight data from the F-15B/836 research airplane. Ding and Li (2007) proposed an online SHM method for a long-span suspension bridge, using the wavelet packet energy spectrum (WPES) method. They decomposed the structural responses excited by ambient vibration using the wavelet packet analysis, and detected the change of conditions based on the energy of the component signal. Using wavelet transformation to pre-process operating signals for SHM systems has developed rapidly, and it will find more applications in the future.

$\mathrm{S}$ transformation was an extension of the continuous wavelet transformation (CWT). It was introduced by Stockwell as a "phase correction" of CWT. S transformation is based on a moving and scalable localizing Gausssian window. It can provide frequencydependent resolution, while at the same time maintaining a direct relationship with the Fourier Spectrum (Stockwell 1996). Pakrashi and Ghosh (2009) explored the possibility of applying S transformation to detect the system's characteristic changes in the presence of noise, and validated its performance by presenting successful identifications of a sudden change in stiffness of a one degree freedom system, and an open crack on a beamlike structure. Wang and Tansel (2007) used S transformation to estimate the propagation features of lamb waves on a plate structure, and identified the occurrence of damages by the delay time and echoes. They also used inverse $\mathrm{S}$ transformation and genetic algorithm to reconstruct the response signal. $\mathrm{S}$ transformation has the desirable characteristics that continuous wavelet transformation lacks, and it can give a more accurate time-frequency representation of sensory signals (Stockwell 1991). S 
transformation is a promising method, and it will see more applications in the field of SHM.

\subsubsection{Decision Making}

The other important issue of SHM is to determine the condition of the system according to the features extracted in the previous process. Different applications have specific approaches for decision making. Model-based reasoning systems, rule-based expert systems, case-based reasoning systems, learning systems and probabilistic reasoning systems are examples of the many diverse approaches (Patterson-Hine et al. 2005). To take advantages of these reasoning systems, they are often integrated together to evaluate the condition of the system.

Model-based reasoning describes utilizing functional engineering models to estimate and predict the health state of systems (Patterson-Hine et al. 2001). In this approach, the structural model of the system is developed based on the understanding of the system, and the effect of damage may find an expression in the model (Fritzen 2005). When it is applied for SHM, the measured state of 
structural integrity will be compared with the estimation of the state by the model, and the discrepancy may indicate the occurrence of damages and provide a measure to isolate damages (Tumer and Bajwa 1999). Poll and Iverson (2003) created compositional models of the main shut-off valve of Hybrid Combustion Facility (HCF), POV-4, including both the physical structure and the operational behavior of the system. They compared the diagnostic performance of TEAMS, Livingstone2, and RODON using logged data of HCF built at NASA Ames Research Center, and found model based reasoning could strengthen Integrated Vehicle Health Management (IVHM) applications. Smith and Kripakaran (2008) explored the multiple model approach to address different assumptions of bridges, and illustrated how this approach could optimize the positioning of sensors. Patterson-Hine et al. (2001) developed a real-time, model-based reasoning system, using TEAMS-RT real-time diagnostic engine, and validated it on the UH-60 Rotorcraft Aircrew Systems Concepts Airborne Laboratory (RASCAL) flight research aircraft. 
The performance of the model based diagnosis depends on the accuracy of the system model. However, for complex systems, the accurate model is either not available due to lack of fully understanding of the system or too complicated to be applied for SHM.

Case based reasoning (CBR) resembles human reasoning and problem solving processes, which solves new problems based on the specific solutions of similar previously experienced situations (Stumptner et al. 2009). The problem solving cycle of CBR includes four phases: Retrieve, Reuse, Revise and Retain (Aamodt and Plaza 1994). It retrieves similar problems stored in the case base and reuses the solutions. It revises the solutions when needed, and the new information obtained is stored for future use. CBR is rapid and cost effective, since it does not have to develop new solutions (Freudenthaler et al. 2008). CBR has been widely applied in SHM. Mujica et al. (2008) utilized cased based reasoning to identify and locate impact on part of a commercial aircraft wing flap. The case base was established by the location of the impact and the according 
principle features extracted by wavelet coefficients. Yang et al. (2009) established a reference pattern of a cable-stayed bridge under its healthy condition with a set of deflection data collected by sensors embedded at critical checkpoints. This was then compared with the current deflection pattern to identify the abnormal condition. CBR also saw applications on evaluating health conditions of turbines (Devaney and Cheetham 2005; Georgin et al. 1995) and gear boxes (Olsson et al. 2004). However, the disadvantage of this approach for SHM is that there is a lack of previous damage cases, and it may be difficult to determine the relevance among problems.

Rule based expert systems model how a human expert analyzes a particular situation based on expert knowledge to provide answers, predictions and suggestions (Abraham 2005). The expert knowledge is represented in the form of a set of if-then rules, which consists of the conditional statements relating to a bunch of facts (Buchanan and Duda 1982). Rule based reasoning is suitable to solve problems for which expertise and experience are available but an accurate 
model of the system is difficult to obtain, or too complicated for real time applications (Patterson-Hine et al. 2005). Schein and Bushby (2006) established a knowledge base with 28 rules derived from mass and energy balances to identify five different operating conditions of air handling units. Wang (2000) utilized rule based reasoning and neural network to identify incipient faults of power transformers. The advantage of Rule based reasoning is modularity, uniformity and naturalness, while its disadvantage is the bottleneck problem of acquiring knowledge and the difficulty to represent expert knowledge (Schein and Bushby 2006).

Learning approaches are information-processing technologies which derive concepts, relationships, descriptions or conclusions from a set of operating data (Ke and All 1988). These processes aim to discover rules and patterns from the statistical characteristics of the data and apply the learning to solve new problems (Filipic and Junkar 2000). These data driven approaches accumulates knowledge from learning; the same as human's learning mechanism. Learning 
approaches not only provide plenty of methods for reasoning, but also generate useful information for other reasoning approaches (Patterson-Hine et al. 2005).

There are many algorithms for learning systems. Neural networks, which simulate the information processing by neurons with computational structures, have proven successful in many applications for diagnostic reasoning. Lopes et al. (2000) used artificial neural networks to detect impedance changes to locate loosening bolts on a steel truss bridge joint. Ko and $\mathrm{Ni}$ (2004) applied a hierarchy neural network to detect characteristics changes of a cable bridge and validated the feasibility of this approach by comparing the estimation and the measurement of sensors. Other methods, such as Support Vector Machine (Pawar and Jung 2008), Genetic Algorithm (Chou and Ghaboussi 2001), Self Organizing Map (Li et al. 2009), have also been widely applied for SHM systems.

Different applications have specific needs for diagnostic reasoning; determining the best method for a given monitoring system is critical. In many 
cases, a combination of different approaches may achieve optimal results (Lee

2002; Li and Huang 2009).

CHAPTER III

\section{THEORETICAL BACKGROUND OF DATA PROCESSING}

Data processing is a critical and integral part of SHM. Once the operational evaluation of the design has been passed and the sensor network has been set up, the choice and implementation of techniques to process the data and make the decision are arguably the most crucial ingredient of SHM (Worden and Dulieu-Barton 2004). In this chapter, the theoretical background addressing data processing issue for SHM is outlined. First, methods transforming signals from time domain to frequency domain to extract modal parameters are discussed. Then, algorithms for mapping, classifying and reasoning are presented.

\subsection{Transformation Algorithms}

3.1.1 Fast Fourier Transformation 
FFT is the most widely used method transforming information from time domain to frequency domain. FFT is a computationally efficient algorithm that calculates the Discrete Fourier Transform (DFT) and its inverse. The DFT is defined by the formula (Cooley et al. 1969):

$$
F_{n} \equiv \sum_{k=0}^{N-1} f_{k} e^{-2 \pi i n k / N}
$$

Where, $f_{k}=f(\mathrm{tk})$ is a function of time, with $\mathrm{k}=0,1, \ldots \mathrm{n}-1$. FFT is a quicker version of the DFT because it decreases the number of calculations for $\mathrm{N}$ points from $2 \mathrm{n}^{2}$ to 2nlog2(n) (Duhamel and Vetterli 1990).

The advantage of Fourier Transform is that the frequency domain characteristics of the signals can be easily extracted and analyzed. However, the frequency-domain representation of original signals may lose information relating to time. In many applications, the time-frequency representation (TFR) is needed to identify when the event occurs. To keep the time component of the signals, the short time Fourier transformation (STFT), wavelet transformation (WT) and S transformation (ST) may be used to study the time-varying characteristics of the spectral information.

\subsubsection{Short Time Fourier Transformation}

The STFT includes time domain information by calculating Fourier transforms of short periods of time defined by a window function. As the window slides along the time axis, the Fourier transforms of the entire time series is obtained piece by piece. Assembly of these pieces represents the characteristics of the signal. The STFT can be written as (Paliwal and Alsteris 2005): 


$$
\operatorname{STFT}(\tau, f)=\int_{-\infty}^{+\infty} h(t) w(\tau-t) \exp (-i 2 \pi f t) d t
$$

Where, $h(t)$ is the time series, $w$ is the window function and $\tau$ is the position of the window along the time axis. The optimization of the window width determines the compromise of the frequency and the time resolution of the analysis. A wide window gives relatively better frequency resolution, while a narrow window gives better time resolution (Niethammer et al. 2001).

Since the fixed window size limits both the frequency and the time resolution, WT, ST and other Fourier derived methods (Belkic et al. 2000) have been developed to adapt window width in function of the frequency to achieve optimal resolution. These multiresolution algorithms can achieve good time resolution at high frequencies and good frequency resolution at low frequencies.

\subsubsection{S Transformation}

S transformation was introduced by Stockwell (1996) as an extension of continuous wavelet transformation (CWT). It is defined as a "phase correction" of CWT,

$$
S(\tau, f)=\exp (i 2 \pi f \tau) W(\tau, d)
$$

Where,

$$
W(\tau, d)=\int_{-\infty}^{+\infty} h(t) w(t-\tau, d) d t
$$

is the CWT of a function $h(t)$. Thus, the S transform is defined as (Stockwell 1996):

$$
S(\tau, F)=\int_{-\infty}^{+\infty} h(t) \frac{|f|}{\sqrt{2 \pi}} e^{-\frac{(\tau-t)^{2} f^{2}}{2}} e^{-i 2 \pi f t} d t
$$


$\mathrm{S}$ transform is based on a moving and scalable localizing Gausssian window, and it can provide frequency-dependent resolution, while at the same time maintain a direct relationship with the Fourier Spectrum (Stockwell 1991).

\subsection{Backpropagation Neural Network}

Neural networks are a series of computational algorithms, which include many diverse models and approaches. The goal of neural networks is to emulate a human's problem solving process. The basic idea of a neural network is to construct a neuronal structure which consists of neurons and synapses (connections between neurons), and learn patterns presented to it by altering the strengths of connections between neurons or changing the connections themselves. The knowledge learned by neural networks is stored in the neurons and their connections, when needed, it can be processed and passed along the neural network.

Backpropagation neural networks (Rumelhart et al. 1986) are the widely used supervised learning algorithm, which was first implemented in 1986. The structure of the backpropagation neural network includes three layers of neurons, the input layer, the hidden layer and the output layer. An example of the backpropagation neural network is presented in Fig.2. 


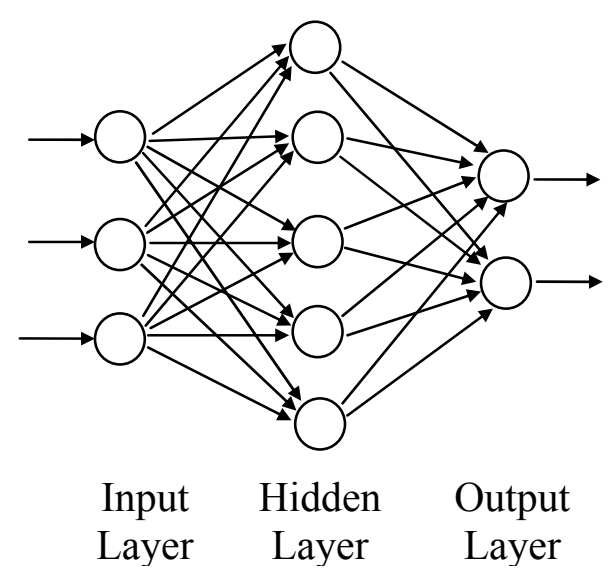

Fig.2 Structure of a Backpropagation Neural Network

The training of backpropagation neural network is to adjust the exciting thresholds of neurons and the connections between neurons, so that the prediction errors varied from the learning target can be minimized. It is an iterative process including the following steps: estimating output using forward propagation, calculating errors of estimated output and targets, propagating errors backward and updating weights.

First, the estimated output of the neural network is calculated using forward propagation:

$$
\boldsymbol{y}=f(\boldsymbol{x}, \boldsymbol{w})
$$

Where, $\boldsymbol{y}$ is the estimated output vector. $\boldsymbol{x}$ is the input vector from the training data set, and $\boldsymbol{w}$ is the weight vector indicating the strength of the connections between two neurons. When the input signal propagates through the neural network, the information stored in each neuron of the hidden layer and the output are calculated based on the estimations of previous layer neurons connecting with the current neuron, and the weights of the connection.

Then, the error (the distance and the direction) between the estimated output and the given target $z$ from the training data set is computed, 


$$
\delta=z-y
$$

The error will then propagate backward through the neural network to evaluate the distribution of the error in the network structure. The error for each neuron of the hidden layer and the input layer can then be determined by:

$$
\boldsymbol{\delta}^{\prime}=g(\boldsymbol{\delta}, \boldsymbol{w})
$$

Finally, the weight of each connection will be updated,

$$
\boldsymbol{w}^{\prime}=\boldsymbol{w}+\eta h\left(\boldsymbol{\delta}^{\prime}, \boldsymbol{x}\right)
$$

Where, $\eta$ is the learning rate.

The procedure will be repeated until the error satisfies the requirement of accuracy.

The learning process of backpropagation neural network is to propagate backward the prediction error between the learning target and the estimated output in order to update the value of neurons and the connection weights, so that the error of the estimated output can be minimized. If the network is properly trained, it can learn the pattern that relates the input variables to the output variables, and can subsequently be used to evaluate the new coming input with the information stored in neurons and the weights of connections.

\subsection{Self Organizing Map}

The Self Organizing Map, also called Kohonen map, was first introduced by Teuvo Kohonen. SOM is an unsupervised neural network which can be trained to represent data via a competitive learning strategy. The most significant feature of SOM is its extensive visualization capability (Penn 2005). It is difficult for humans to see through high dimensional data. SOM can project high dimensional input vectors into low dimensional feature maps, which can facilitate the visualization of the relationship of input data. The way SOMs go about reducing dimensions is to first characterize the topological relations 
of the high dimensional dataset; the topological constraint can then cluster the similar data together when they are projected onto a low dimension map (Kohonen 2001). Thus, the inherent relations of the data elements can be preserved for visualization. The SOM is a popular tool to analyze multidimensional, non-linear or highly noisy signals (Cottrell 2006). In fact, any dataset with definable distance metrics can be mapped with a SOM. Another advantage of SOMs is they require no prior knowledge of the input dataset to be analyzed (Kohonen 1997). In addition, SOM networks are easy to implement and efficient in computation.

The basic structure of SOM networks consists of an input layer and an output layer. The neurons of each layer are fully connected, and each neuron of the output layer is associated with a reference vector, which connects between the input and output layers. The connections (weights), describe the properties of neurons. Since the reference vectors connect each input vectors to output neurons, the dimensionality of the weights are the same as the input vectors. The output layer is usually a two dimension layout, with the topology of rectangular, hexagonal, or even irregular organization. The size of the output map, which is defined by the number of neurons, should be decided by the specific application. The bigger the map, the more details illustrating the characteristics of input data. The suggested number of neurons can be $5 \sqrt{n}$, where $n$ is the number of input data point (Watts and Worner 2009). Fig.3 shows an example of the input multi dimensional data and the layout of the output neurons. 


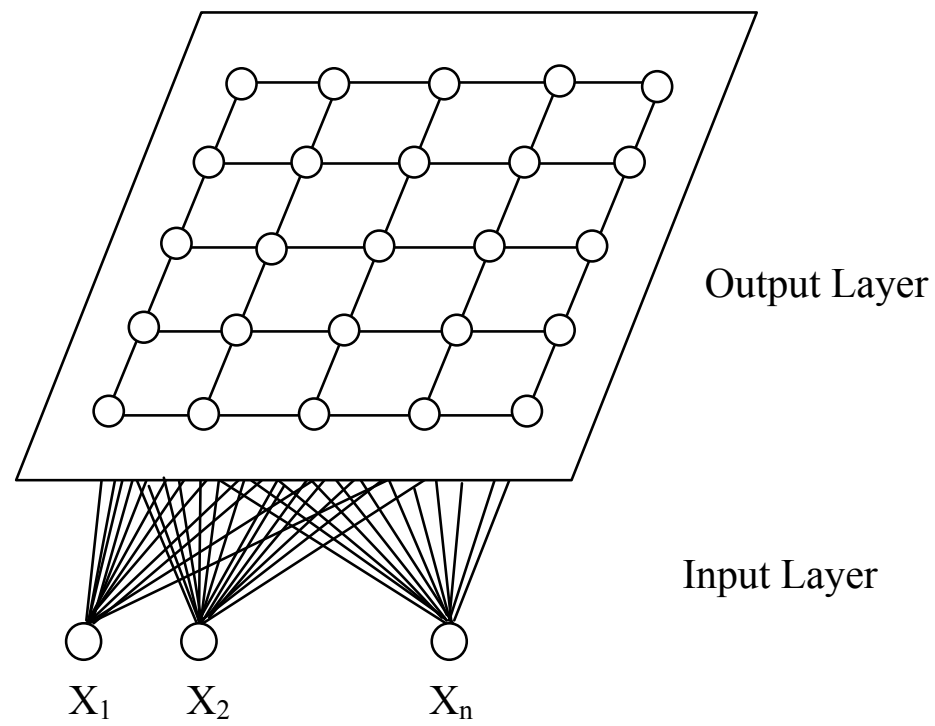

Fig.3 Structure of a Self Organizing Map

The learning process of SOM is iterative:

First of all, the reference vectors associated with the neurons have to be initialized. Generally, the reference vectors are initialized randomly, but they can also be normalized through dividing each element of the vector by the sum of their elements.

Neurons of the output layer will then compete with each other to match the input data presented to the SOM. The distance between the input vector and each output neuron is calculated according to the discriminate function. The specific neuron which has the smallest distance is considered to be the winner representing the input data the best. Generally, the Euclidean distance is calculated using the following equation:

$$
\left\|x(t)-w_{i}(t)\right\|={ }_{j}^{\min }\left\{\left\|x(t)-w_{j}(t)\right\|\right\} \quad j=1,2, \ldots, n
$$

Where, $x\left(x_{1}, \ldots, x_{n}\right)$ is the input vector from the input training set and $w_{j}\left(w_{j l}, \ldots, w_{j n}\right)$ is the weight vector of the network. Index $i$ represents the winner neuron and $t$ is the index of the regression step. 
The network weight vectors will then be updated. The weights of the winner and the neurons within the neighborhood of the winner are modified so that they can represent the input sample better. Weight vectors of the neurons outside the neighborhood will not change.

The iterative update strategy of the weight is:

$$
w_{j}(t+1)=\left\{\begin{array}{lr}
w_{j}(t)+\eta(t) h_{j i}(t)\left\|x(t)-w_{j}(t)\right\| & j \in N_{i} \\
w_{j}(t) & \text { otherwise }
\end{array}\right.
$$

Where, $N i$ indicates the neighborhood, and weights of neurons inside this boundary will be updated according to the above equation. $0<\eta(t)<1$ is the learning rate, which decreases monotonically during the learning process. $h_{j i}(t)$ is the neighborhood function that defines the closeness of neurons immediately surrounding the winner. It is often chosen as Gaussian:

$$
h_{j i}(t)=\exp \left(-\frac{d^{2}}{2 \sigma^{2}(t)}\right)
$$

Where, $d$ is the distance between the winner and the current neuron $j$, and $\sigma$ corresponds to the neighborhood width, which decreases with the regression steps.

With these modifications, the winner and its neighborhood make themselves look more like the sample data, while the neurons far away from the winner will not learn the attribute of the input data. The "winner-take-all" selection rule strengthens the learning of the inherent features of the input vectors. As time progresses, the SOM will achieve the convergence.

The learning process of SOMs shows that the output neurons reflect the statistics characteristics of the input dataset through maximizing the similarity to the input patterns 
and minimizing the dissimilarity during the learning. In this manner, each neuron in the SOM can recognize data similar to itself. The self organizing characteristic of SOMs can be utilized to cluster and classify data of different categories.

\subsection{Fuzzy Logic}

Fuzzy logic refers to the method that making decisions in an approximate way similar to the way humans do. It uses fuzzy and linguistic labels, rather than quantitative values to represent and manipulate variables (Zadeh 1975). The variables are allowed to be true, false or anywhere in between, therefore modeling problem solving of the human approach. The process of fuzzy logic system includes the following (Bickraj 2006):

Normalization $\rightarrow$ Fuzzification $\rightarrow$ Inference $\rightarrow$ Composition $\rightarrow$ Defuzzification $\rightarrow$ Denormalization

Fig.4 Process of a Fuzzy Logic System

The normalization and denormalization steps can be skipped according to different applications. The purpose for normalization is to provide unit input which can reduce the calculation burden for later steps.

Fuzzification is the process to convert crisp values of input variables to fuzzy grades indicating the degree of membership of each category represented by membership functions. Membership functions are extracted from humans' expert knowledge about the interested system, and they can indicate the degree of truth of the input variables. Thus, a variable may be labeled partially as a member of a set, rather than whether belongs to a set or not. The membership functions can be triangular, trapezoidal, singleton or variations of the Gaussian function, etc. There are input member functions and output member functions to testify the membership degree for input and output. 
In the inference step, humans' expert knowledge about the dynamic system provides a set of rules for fuzzy logic to make decisions. The fuzzy rules are expressed in the format as: If $\mathrm{x}$ is $\mathrm{A}$ Then $\mathrm{y}$ is $\mathrm{B}$. The membership of the premise set $\mathrm{A}$ is calculated for input $\mathrm{x}$, then according to the rule, the membership of the conclusion set $\mathrm{B}$ will be calculated for output $y$. These calculations will be carried out for each of the rules. The more the rules, the better the performance. However, more rules will decrease the computational efficiency.

Composition means the combination of all the memberships calculated for each output variable according to all rules. Then, a final fuzzy value will be assigned for each output variable. There are several methods to implement composition. Min-Max is a frequently used method. It clips off the membership function for output variables at a height corresponding to the partial membership of the input and takes the point-wise maximum of all the fuzzy degree of the memberships assigned to the output (Kundu 1998).

Finally, defuzzyfication reconstructs the crisp value based on the result of composition process. Gravity center is a popular way to defuzzyfy the membership value of output variables, which calculates the center of the area limited by the combination of all the according membership functions. Weighted average method, mean-max membership method, and max membership method are examples of so-called Maximum methods (Chandramohan et al. 2006). These methods determine the crisp value for the output variable based on a maximum membership value of certain category.

\subsubsection{Mamdani and Sugeno Methods}


There are two different inference types of fuzzy logic. One is the Mamdani method, which is the most commonly used fuzzy inference technique. The other one is Sugeno method. The difference of these two is the way how membership functions representing the degree of truth for the conclusion output (Jassbi et al. 2006). In Mamdani method, the output's membership of varied categories will be combined, and the centroid of a twodimensional shape covered by according membership functions has to be calculated. The integration of an area bordered by continuously varying functions imposes substantial computational burden. To solve this problem, Sugeno suggested using a single spike, a singleton, as the membership function of output variables, which makes Sugeno method computationally effective. The format of the Sugeno-style fuzzy rule is (Sugeno and Yasukawa 1993):

\section{IF $\mathrm{x}$ is $\mathrm{A}$ AND $\mathrm{y}$ is $\mathrm{B}$ THEN $\mathrm{z}$ is $\mathrm{f}(\mathrm{x}, \mathrm{y})$}

Instead of a fuzzy set, Sugeno method uses a mathematical function of the input variable to represent the rule consequent. The most commonly used Sugeno output membership functions are linear and constant.

As a problem-solving methodology, fuzzy logic has been utilized widely in control systems. It also finds a wide range of applications in other fields where decision making needs to be carried out. Fuzzy logic provides a simple way to derive a definite conclusion based upon imprecise, noisy, or missing input information. In addition, the hardware and/or software to implement fuzzy logic can be simple. The inherent robustness of fuzzy logic enables it work well even though the membership functions and the rules are roughly designed. The performance of fuzzy logic can be improved conveniently through 
optimizing the membership functions and inference rules with genetic algorithms and neural networks.

\subsubsection{Neuro-Fuzzy System}

The disadvantage of fuzzy logic lies in that it cannot automatically acquire the membership functions and the inference rules. And once they are determined, it is errorprone and time-consuming to tune the parameters of the fuzzy system. However, for neural network, it is difficult to analyze the trained network and explain how neural networks get their conclusions (Nurnberger and Kruse 1999).

Since in theory, neural networks and fuzzy logic systems are usually convertible to solve the same problem, these two methods can be combined to overcome the limitations, and take advantage of the learning ability of neural networks and the interpretability of the rule-based fuzzy systems (Masuoka et al. 1990).

There are different kinds of combinations of neuro-fuzzy systems. Improving the performance of neuro-fuzzy systems using neural networks and genetic algorithms to learn fuzzy rules and tune membership functions is widely used (Lei and Wang 2004). In this sense, a neuro-fuzzy system is a fuzzy system that can determine or optimize its parameters (fuzzy sets and fuzzy rules) based on the learning algorithm derived from neural networks trained by target samples (Stieler et al. 2009).

\subsection{Dynamic Model of a Four Rotor Helicopter}

Small four rotor helicopters have gained popularity for unmanned surveillance operations. They can achieve quasi-stationary hovering in addition to vertical take-off and landing (VTOL) in limited space. Unlike regular helicopters, they are underactuated 
dynamic vehicles with four fixed pitch angle rotors (McKerrow 2004). The motion of the helicopter is controlled through rotating the front and rear rotors counterclockwise while the left and right rotors clockwise. Using four rotors increases the payload capacity and the maneuverability of the helicopter.

The propulsion of the four rotor helicopters comes from four electric motors. The lift forces will vary with the rotor speeds. The differences of lift forces will tilt the helicopter, and accelerate the helicopter along that direction. By increasing/reducing the speed of the front rotor while at the same time reducing/increasing the speed of the rear rotor, the pitch movement can be obtained. By changing the speed of the lateral rotors with the similar approach, the roll movement can be obtained. By varying the speed of the two pairs of rotors together, the yaw movement can be obtained (McKerrow 2004).

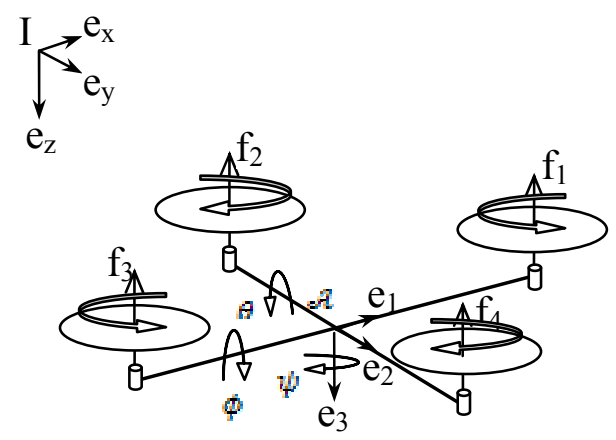

Fig.5 Schematic Diagram of a Four Rotor Helicopter

The diagram (Erginer 2007) of the four rotor helicopter is shown in Fig.5. Where,

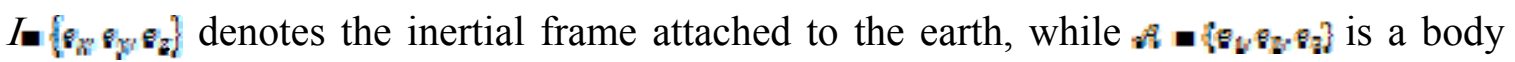
fixed frame attached at the center of mass of the helicopter. $\phi, \theta, \psi$ are Euler angels, which denote roll, pitch and yaw respectively.

The dynamic model of the helicopter is derived as following (Hamel et al. 2002): 


$$
\begin{aligned}
& \dot{\xi}=v \\
& v=g \varphi_{z}-\frac{1}{m} T R_{\varphi_{z}} \\
& A=R a h(n) \\
& k_{\gamma} n=-n \times k_{\eta} n-\theta_{\Omega}+\tau_{n} \\
& u_{r} \omega_{1}=\tau_{1} Q_{i}
\end{aligned}
$$

Where, $T$ is the total thrust,

$$
T=\sum_{\ln }^{+\pi} \| f t
$$

$f_{i}$ is the lift force of rotor $I$,

$$
f=-b w e_{1}
$$

$P$ is the rotational transformation matrix,

$$
R=\left[\begin{array}{ccc}
C_{\phi} C_{\psi} & c_{\psi} S_{\phi} S_{\phi}-S_{\psi} C_{\phi} & c_{\psi} S_{g} C_{\phi}+S_{\psi} S_{\phi} \\
C_{\phi} S_{\psi} & S_{\psi} S_{\phi} S_{\phi}+C_{\psi} C_{\phi} & S_{\psi} S_{\phi} C_{\phi}-C_{\psi} S_{\phi} \\
-S_{\phi} & S_{\phi} C_{\phi} & C_{\phi} C_{\phi}
\end{array}\right]
$$

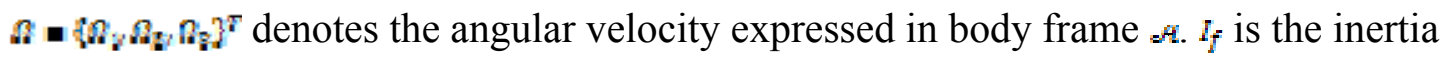
matrix around the center of mass. And

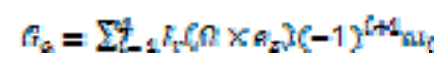

is the gyroscopic torques.

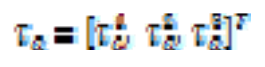

is the airframe torques. And each component of the torque is,

$$
\begin{aligned}
& T h=\text { Wh(w) }-\omega A \\
& \tau_{a}^{2}=d b\left(\omega_{i}^{2}-\omega_{3}^{2}\right) \\
& v_{6}^{1}=\pi\left\{w_{1}^{2}+w_{1}^{7}-w_{2}^{2}-w_{4}^{2}\right.
\end{aligned}
$$

Where, $\kappa$ and $b$ are proportionality parameters depending on the density of air, the properties of rotor blades and other factors. $d$ is the distance from the rotors to the center 
of mass of the helicopter. $w_{i}$ denotes the angular velocity of the rotor $t$. And $i_{z}$ is the moment of inertia of a rotor around its axis. 


\section{CHAPTER IV}

\section{INTRODUCTION TO INDEX BASED REASONING}

\subsection{Index Based Reasoning}

The IBR system is a simple and flexible reasoning system developed for monitoring structured health conditions of complex system. In IBR systems, health conditions of structures are represented with metric indexes. The quality evaluation with HIs enables the IBR to identify and classify the structural integrity effectively. In addition, the resolution of IBR can be adapted in accordance with specific applications. IBR systems can also integrate with other reasoning systems to guarantee the reliability and safety operation of the system.

IBR was first introduced in Florida International University in 2005 (Tansel et al.

2006). It was first developed to detect mishaps in the structures of small UAVs. At that time, it was only a simple LUT based diagnostic system. However, it allowed engineers to customize HI values with training by experimental data using a graphic user interface programmer. Then, vibration based health monitoring and sweep sine wave based monitoring with FFT analysis were introduced into IBR systems. The performance of IBR was proved by the simulations of a HDA and a four rotor helicopter, etc. by Matlab/Simulink models. The neural network training was also added to IBR system. The IBR system was then capable of learning the characteristic patterns after training with the sensory data of the system operating under different health conditions. Later, selflearning and limited interpolation capabilities were embedded into IBR systems to create an easy-to-use health monitoring system, which requires a minimum number of 
experiments for data collection. Finally, IBR systems were explored to evaluate the structural integrity with Lamb wave based health monitoring method.

The proposed IBR system is a hierarchy and distributed network (Tansel et al. 2006). The structure of the IBR system varies according to the specific system to be monitored. Based on the concept of total health management, the issue of SHM should be addressed from the design stage. Thus, the IBR system and according sensor network should be determined based on the design of the system. An example of the IBR system deployment designed to monitor the structure of the Global Hawk is shown in the following diagram.

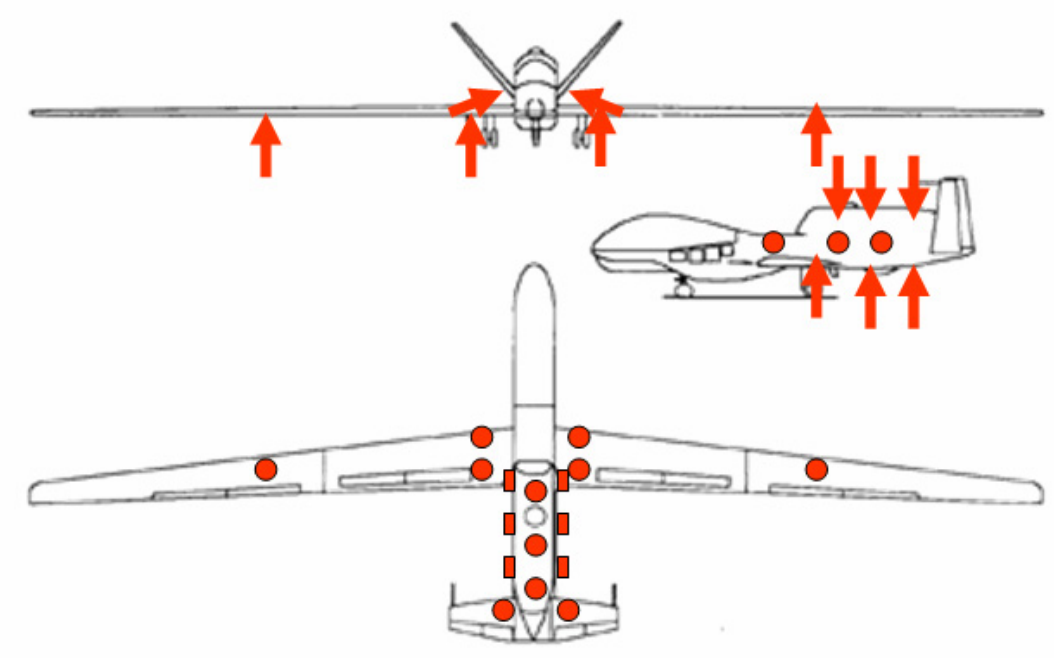

Fig.6 Example of the IBR System

Depending on the complexity of the interested system, the IBR system is composed of just one or thousands IBR nodes (IBRs). These IBRs are distributed around the system to monitor the health condition of specific structures. They are arranged in layers, in which IBRs are grouped to one or several sub-systems. In the previous example, there are 
sub-systems of IBRs monitoring the structural integrity of the wings, the tail, and the fuselage.

IBR nodes are basic processing units of IBR systems; they can acquire and analyze incoming signals, and estimate the local structure integrity with a metric HI. The HI is a diagnostic code if the IBR node is on a higher layer and monitors part of a subsystem. The HI will then be carried up to the next layer and presented to the IBR node connected with the current node as an input. The IBR node of the highest level will collect all the HIs reported from the lower levels to determine the health condition of the whole system. Through this way, the IBR network does not have to transmit raw data; only the estimation of HIs is transmitted along the wires. This will greatly reduce the wire needed, and it can increase the transmit speed. The diagram of IBR systems is illustrated in Fig.7 (Tansel et al. 2006).

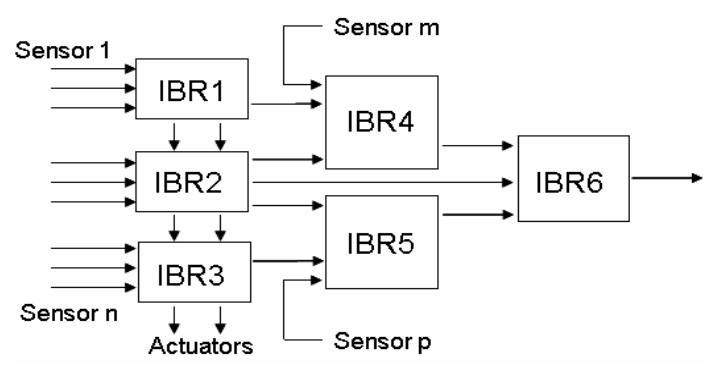

Fig.7 Structure of the IBR Network

Each smart node of IBR system is made up of one or several microcontrollers and sensors. Depending on the characteristics of the sensory data, there are two different types of IBRs. For the simple IBRs, their microcontrollers will extract features of the input signals and classify them directly. However, if the dynamic signals are complicated, the IBR may need an encoder to represent the data in a very compact and meaningful way. In addition, an Index History Evaluator (IHE) may be used to investigate the variation of 
the signal during a time frame to make sure not to send wrong index values because of noise or transition. The simple and advanced IBRs are presented in the following graph (Tansel et al. 2006).

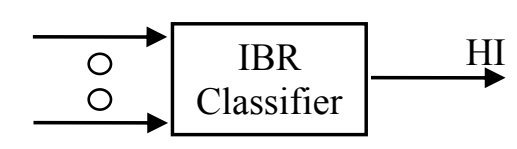

Simple IBR with a Classifier

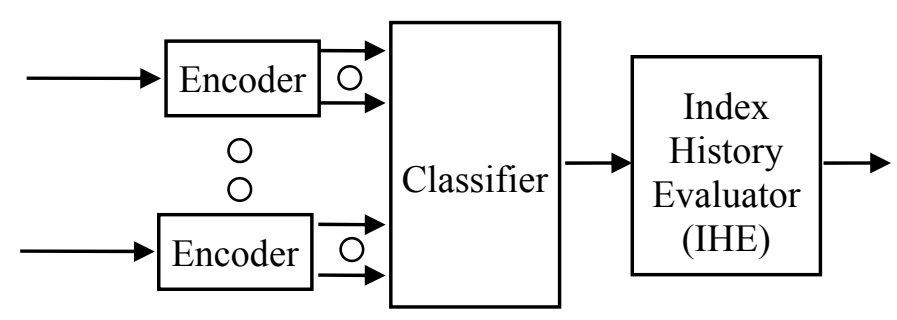

Advanced IBR with a Classifier, Encoder(s), and/or IHE

Fig. 8 Simple and Advanced IBRs

The IBR nodes in different levels also target on different tasks:

- Low-Level IBR nodes

The main purpose of low-level IBRs is to execute feature extraction and pattern recognition. That is, to process sensory signals, extract distinguished features, identify damages, determine local HIs, and send HIs to upper layers.

For IBRs in this level, FFT and other transformations are utilized to analyze the dynamic signals and extract frequency features of the local structures. Neural network and fuzzy logic methods are used to identify, locate and estimate the damages of local structures and determine the local HIs.

- High-Level IBRs 
The main task for high-level IBRs is to assess the situations of the subsystems and the whole system. Further decisions will be made to optimize the parameters of the control system based on the health condition of the whole system. After getting all the HIs sent by low-level IBRs, the main processor of high- level IBRs will assign different weights to local HI. Genetic Algorithm, Neural Network and Fuzzy Logic will be used to set up the relationship of the local HI and the health condition of whole system, and a final decision of the condition of the system will be made

The IBR system has the following advantages:

- The cost of implementing the IBR system is very low compared to current reasoning systems. It requires much simpler hardware.

- It transmits information instead of data, so it reduces the number of wires and increases the transmission rate in the vehicle.

- The fault tolerance of the system can be adjusted by using multiple smart nodes.

- Visual and interactive software platforms make updating LUTs very convenient.

- It is easy to embed new tools to extract features and make decisions. Each node can use different methods. Not only supervised methods, but also unsupervised neural networks can be used.

- It simplifies the training results of neural networks and other pattern recognizing methods to evaluate systems' conditions with a HI.

- IBR can also be customized by the user for specific applications through programming a simple user interface or training with experimental data. 
These advantages make the IBR system a perfect fit for the low-cost UAVs embedded with smart sensors of self-calibration and self-test capability. The IBR system can also be a backup health monitoring system for aircraft and advanced Space Utility Vehicles (SUV).

\subsection{Graphic User Interface Programmer for IBR}

The early version of IBR introduced in 2005 was only a simple LUT based diagnostic system. The advantage of this version is the graphic user interface programmer, through which engineers can program the IBR nodes conveniently. To simplify the programming of the graphical user interface and increase the processing speed of IBR nodes, we recommend having a maximum of three inputs for each node. The following picture shows an example of the graphic user interface to program the IBR node.

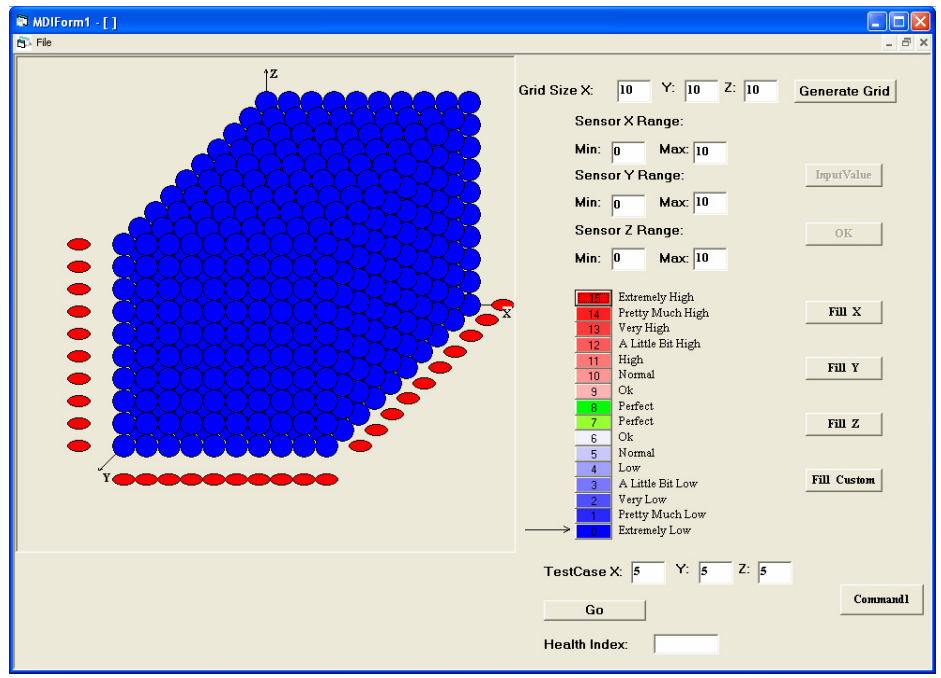

Fig.9 Graphic User Interface of the 3D IBR Programmer

This programmer helps engineers to train the IBR node to learn the characteristic patterns of the experimental data, and then use these patterns as a LUT to estimate the new coming sensory data. Input data collected through different sensors are represented 
by $\mathrm{X}, \mathrm{Y}$, and $\mathrm{Z}$ coordinate for this $3 \mathrm{D}$ programmer. The desired resolution of input data can be obtained based on the grid size and the range of the sensory data. The more the grid, the higher the accuracy in representing the input data. However, it will take more time to process the data. The position of each bubble indicates the according input values of different sensors, which have been normalized and weighted. The color represents the HI value, which is given by the legend.

It is simple and flexible to implement the training of the IBR node. The training can be programmed manually or automatically. Fig.10 a) illustrates the desired responses of manual programming. Each bubble can be filled layer by layer using the color labels representing the health conditions. To program the IBR node automatically, it only needs to click the according button to fill along the specific axis. Fig.10 b) shows an example to automatically fill the IBR node with the input signals collected by sensor Y. Given the range of the input signal, and assumed the HIs changed linearly, the HI values could be filled by interpolation. In most of the cases, the combination of these two ways will help engineers to program the IBR node quickly. Automatically programming can fill batch bubbles while manually tuning can increase the accuracy.
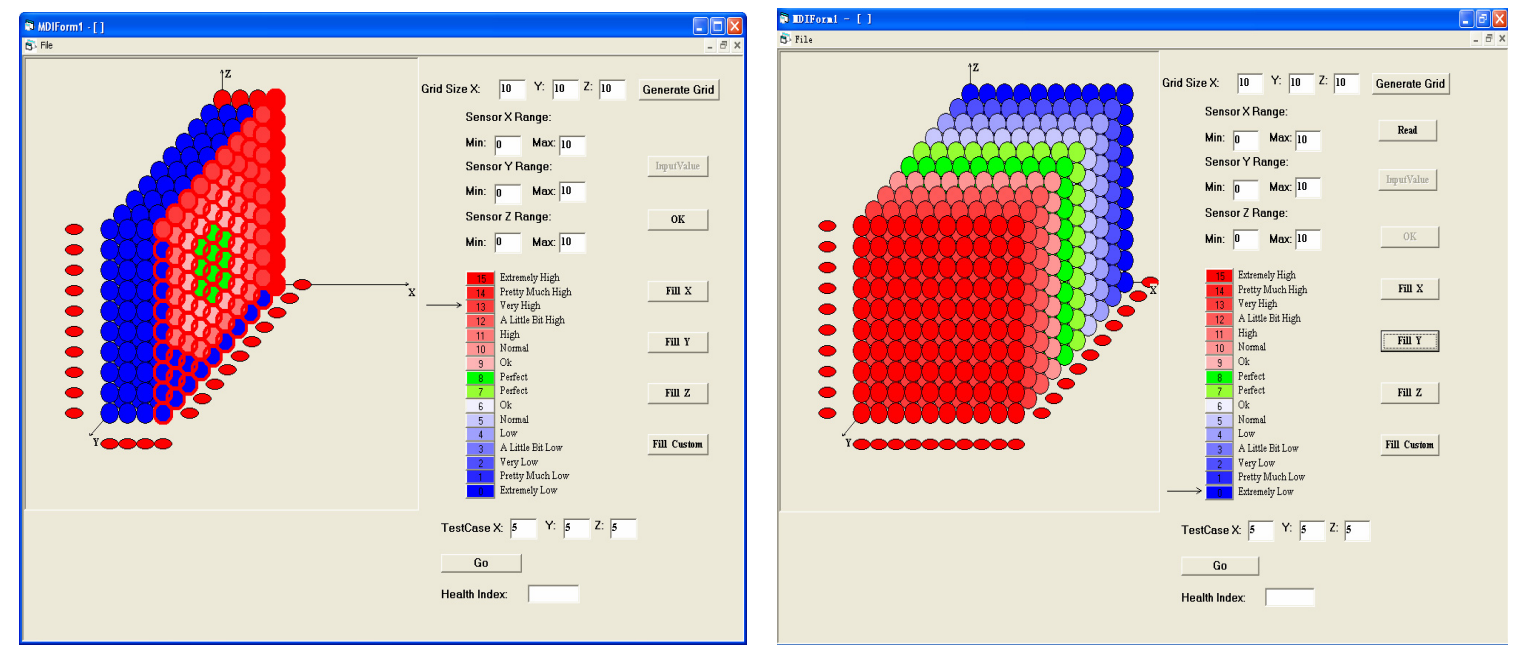

a) Manually
b) Automatically

Fig.10 Programming the IBR node

Other than the simple LUT approach introduced above, the IBR also can integrate other pattern recognition algorithms or optimization methods, such as neural networks, fuzzy logic and genetic algorithms, etc. The above LUT is defined by the engineers, while in the real cases, the pattern of the input data collected by sensors may not be known to engineers. In these cases, feature extraction and pattern recognition technologies need to be applied to train the IBR node to learn the patterns. IBR systems are very flexible to integrate these techniques. The following pictures illustrate how the IBR node is trained with neural network to learn a complicated pattern. The original data is illustrated in Fig.11 a). To learn this pattern, backpropagation neural network program written in visual basic is used. With 15 hidden nodes in the middle layer, the error rate of the training could be as low as 3.9\% percent after 50,000 iterations. Fig. 11 b), c) and d) illustrate the results after iterations of 10,000, 30,000 and 50,000.

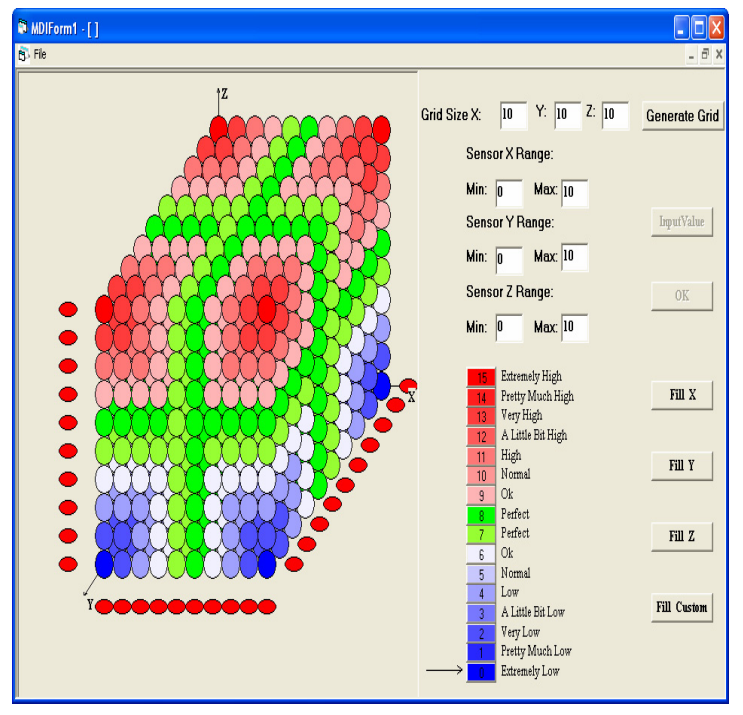

a) Original Pattern

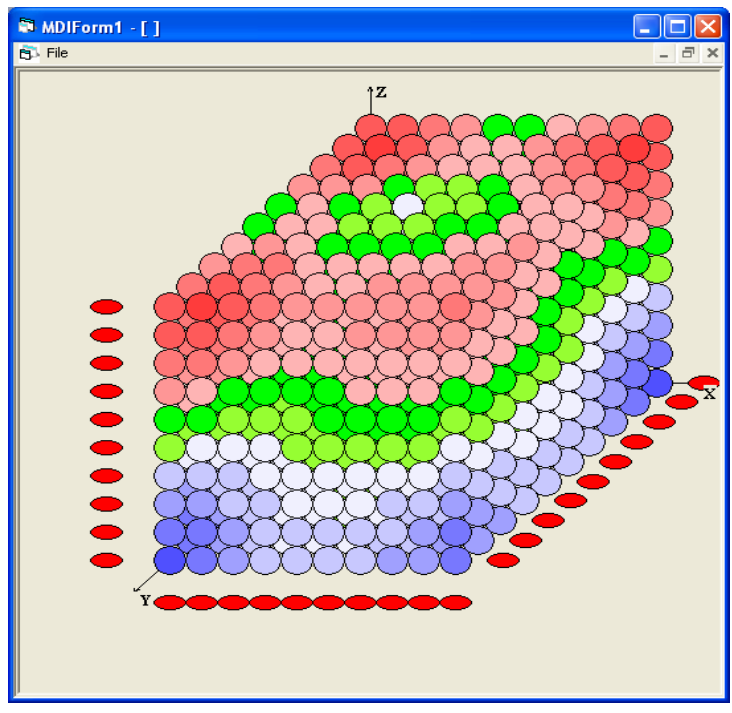

b) After 10,000 Iterations (Err 5.17\%) 
Fig.11 Neural Network Training Results

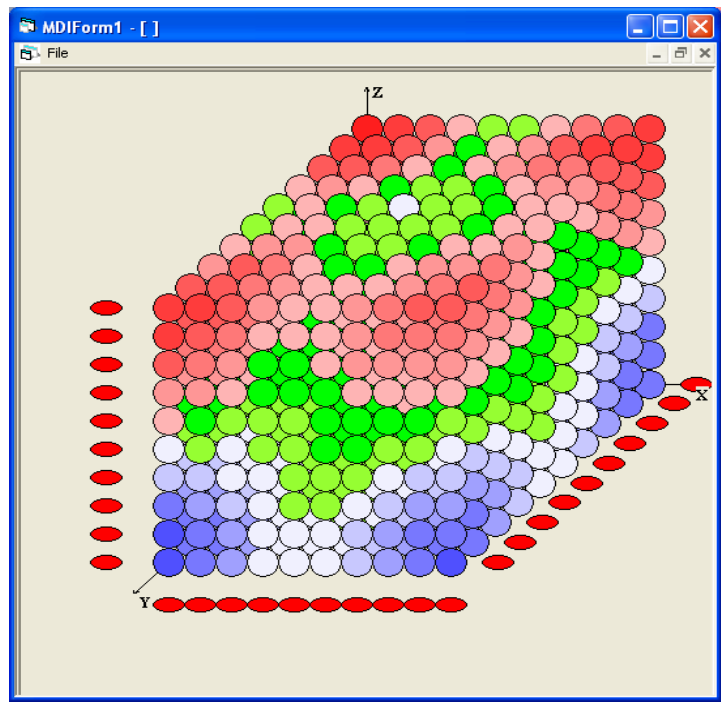

c) After 30,000 Iterations (Err 4.17\%)

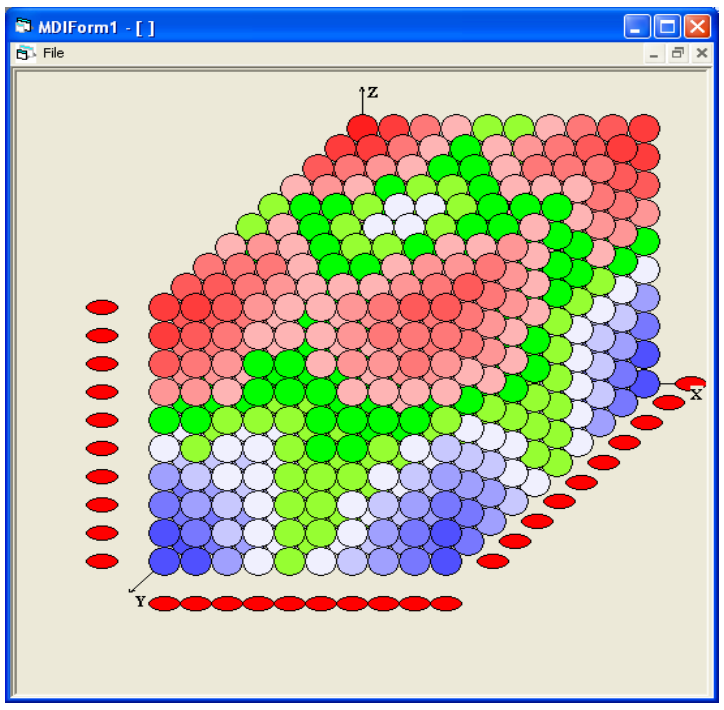

d) After 50,000 Iterations (Err 3.9\%)

Multiple smart nodes like this can construct a hierarchy IBR network. The following diagrams illustrate an example of a two-layer IBR system. Each node in the first layer has three inputs, and it evaluates the local structural integrity based on its classifying algorithm. The estimated HI value is then sent to the node of the following layer, in this case the node of the highest layer, to determine the total health condition of the system. For each node of the IBR system, it can have its own pattern-recognizing methods to classify either the sensory data or HI values reported from the previous level. This greatly increases the flexibility of the data processing part of IBR systems. Feature extraction methods can also be integrated into IBRs to pre-process complex dynamic signals. Fig.12 b shows using FFT to extract the amplitude and the frequency of the sensory data and then present the features to IBR nodes for classifying and identifying. 


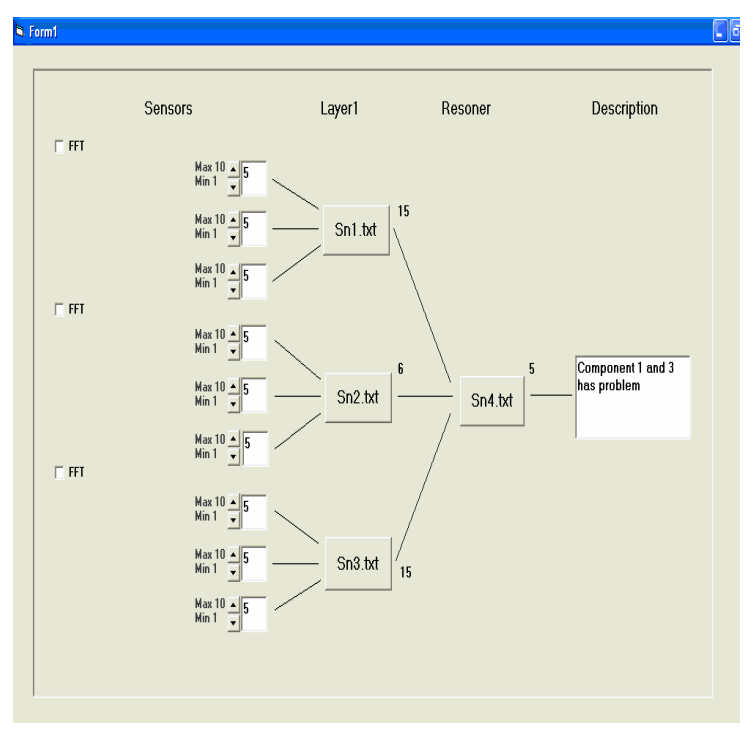

a) Time Domain Input

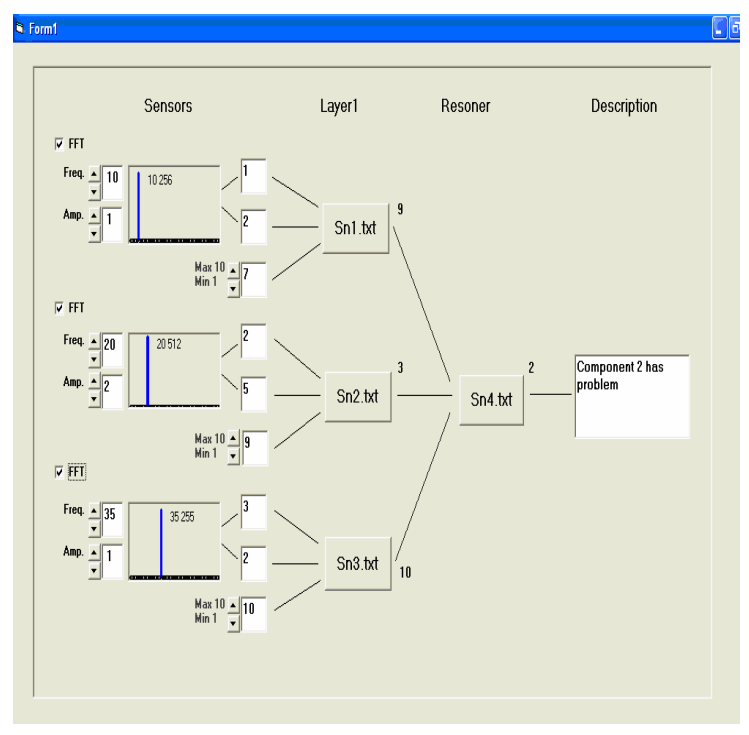

b) FFT Analysis Input

Fig.12 GUI Example of the IBR Structure

\subsection{Single Chip Index Based Reasoner}

When it comes to implement IBR algorithms using microcontrollers, a development environment generating MikroBasic code automatically can be included in IBR systems. The Single Chip Index Based Reasoner (SCIBR) develop environment was created to program Microchip PIC microcontrollers to evaluate the structural integrity of complex systems which need a fault-identifying program ( $\mathrm{Li}$ et al. 2008). The SCIBR can be implemented on a specific Microchip PIC as a stand-alone system using its own clock and $\mathrm{A} / \mathrm{D}$ system. The basic function of SCIBR is to develop a set of rules to determine the boundaries of different health conditions, and used the results to classify the sensory signals. The set of rules were either extracted from the training data set or based on priori knowledge about the system. Engineers can also modify the rules and select the classification criteria according to their expertise in SCIBR environment. Then, a module of MikroBasic code will be generated automatically and ready to be downloaded to 
Microchip PICs. The output of the SCIBR system may be the TTL level signals sent to any external system to indicate the system's conditions or any defect that occurs in the system.

The SCIBR development environment is presented in Fig.13. The user can determine the parameters of the program, such as number of sensors, cases, dimensions of data, and desired HI levels (normal + fault modes). The training data set was collected through the embedded sensors during the operation of the system. The program was trained to learn the boundaries of each component of sensory signals. The HI levels to distinguish the health conditions of the system could be selected by users. The minimum of the HI level is two, including the normal state and one fault mode. Higher index levels are acceptable if, according to the application, it is necessary to detail the different categories of the unacceptable operating states of the system.

The user can also choose the specific classification method among the three available approaches. The first method classifies the sensory data into different health conditions based on the boundaries of each category. The second method calculates the sum of the squared error and utilizes the maximum sum of squared error (MaxSSE) as the classification criteria. The third approach combines the first two methods to estimate and identify the data. 


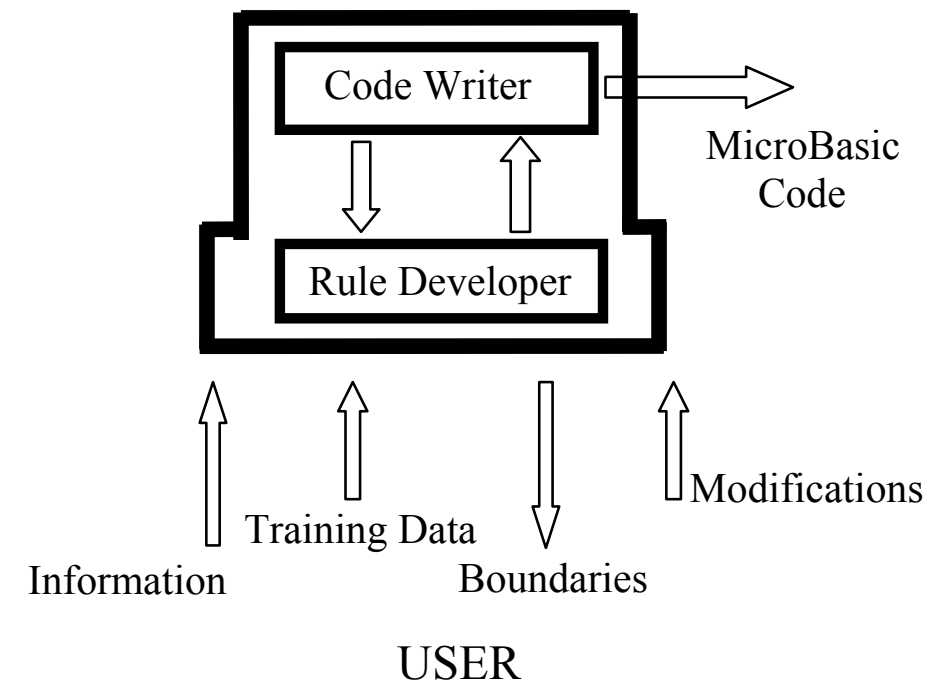

Fig.13 Information Exchange Between the SCIBR Environment and the User Microchip PIC 12F675 was chosen to be programmed and tested due to its tiny size, which is suitable to be packed into the small UAVs or space vehicles, while, its relatively high capability satisfies the requirement to apply SHM using simple IBR alogrithms. However, the program had to be simplified to fit into the barely-enough memory of PIC12F675 microcontrollers. If needed, several pics can be connected to achieve assigned task for specific applications.

The user interface of the system is presented in Fig.14. The user provides information of the training data set such as number of sensors, desired HI levels, data points, and dimension of data. Then, the data file is read into the program and the training progress began. 


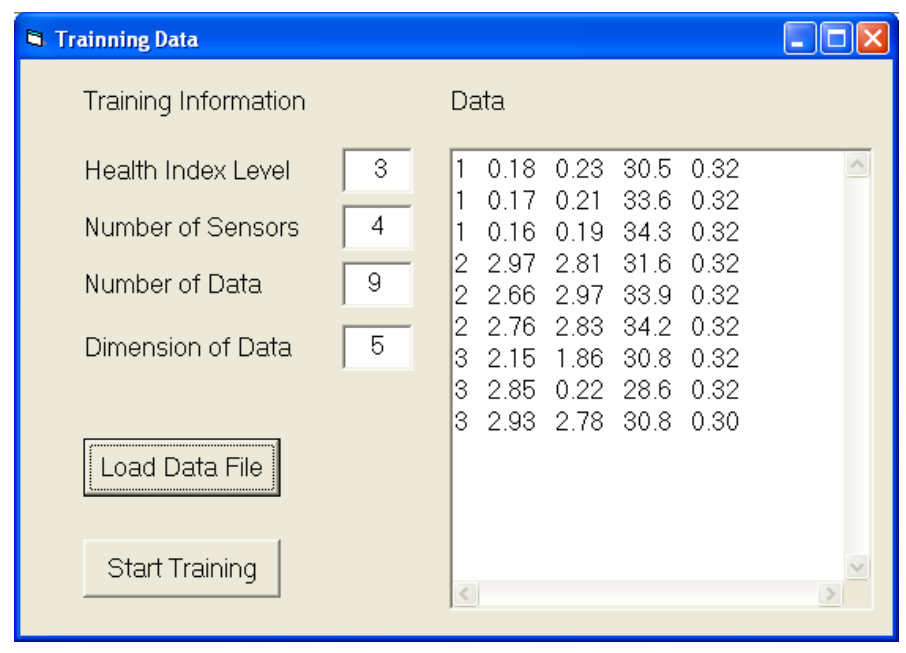

Fig.14 Training Data Input Form

In accordance with the training data acquired by every sensor, SCIBR program determines the boundaries of sensory data to distinguish different categories. Each category corresponds to a certain health condition labeled with an index. If the signal falls into the specific boundary then it belongs to the according HI category. These boundaries are then presented to the user, which is shown in Fig.15, for further correction and modification. MaxSSE is also estimated to provide statistic information about the training data set. All the above parameters can be changed by the user based on the priori knowledge and experience of the system. In this step, the user can also choose the classification method for testing the new coming data. The minimum and maximum values of each category and/or the MaxSSE can be used as the criteria. 


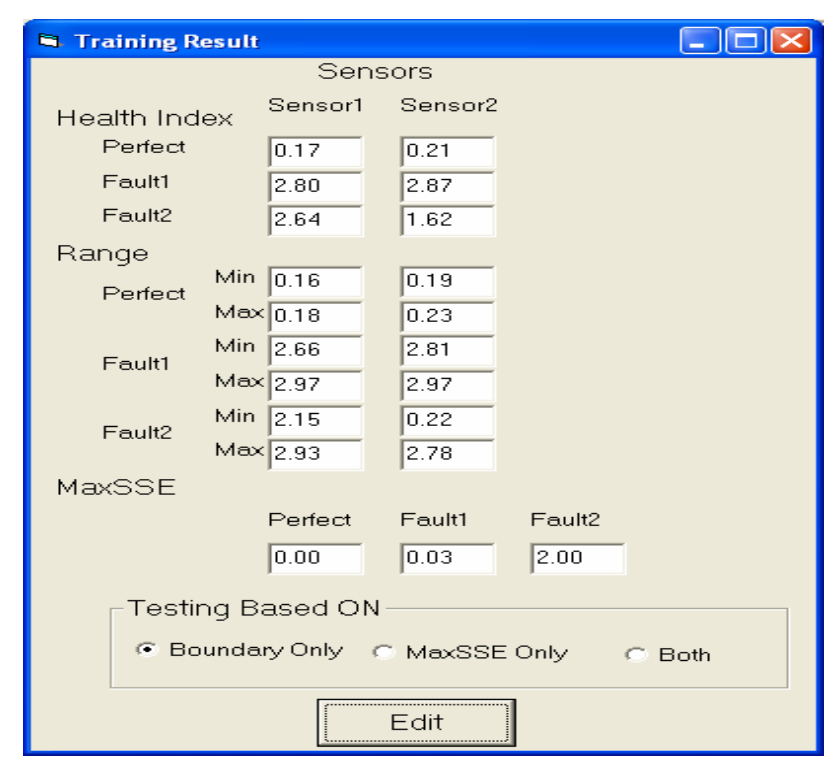

Fig. 15 Boundaries of Signals and Choices of Classifications

The SCIBR programmer can automatically generate the MikroBasic code to implement IBR using microcontrollers. The user may modify the code through the editor of the MikroBasic compiler as shown in Fig.16. The final version of the program module will then be automatically presented to the MikroBasic development environment for compiling and downloading.

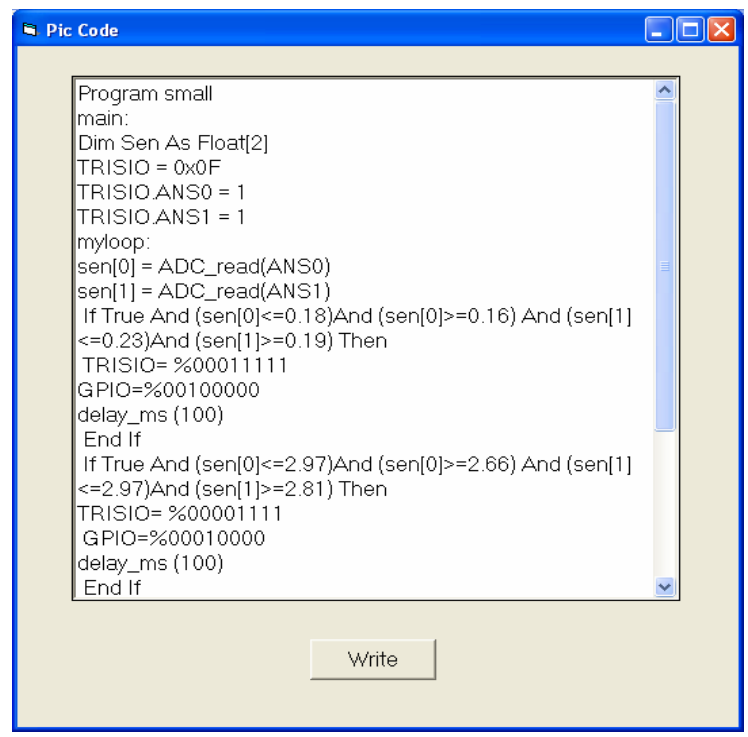

Fig.16 Prepared MikroBasic Code Based on Selected Classifications 


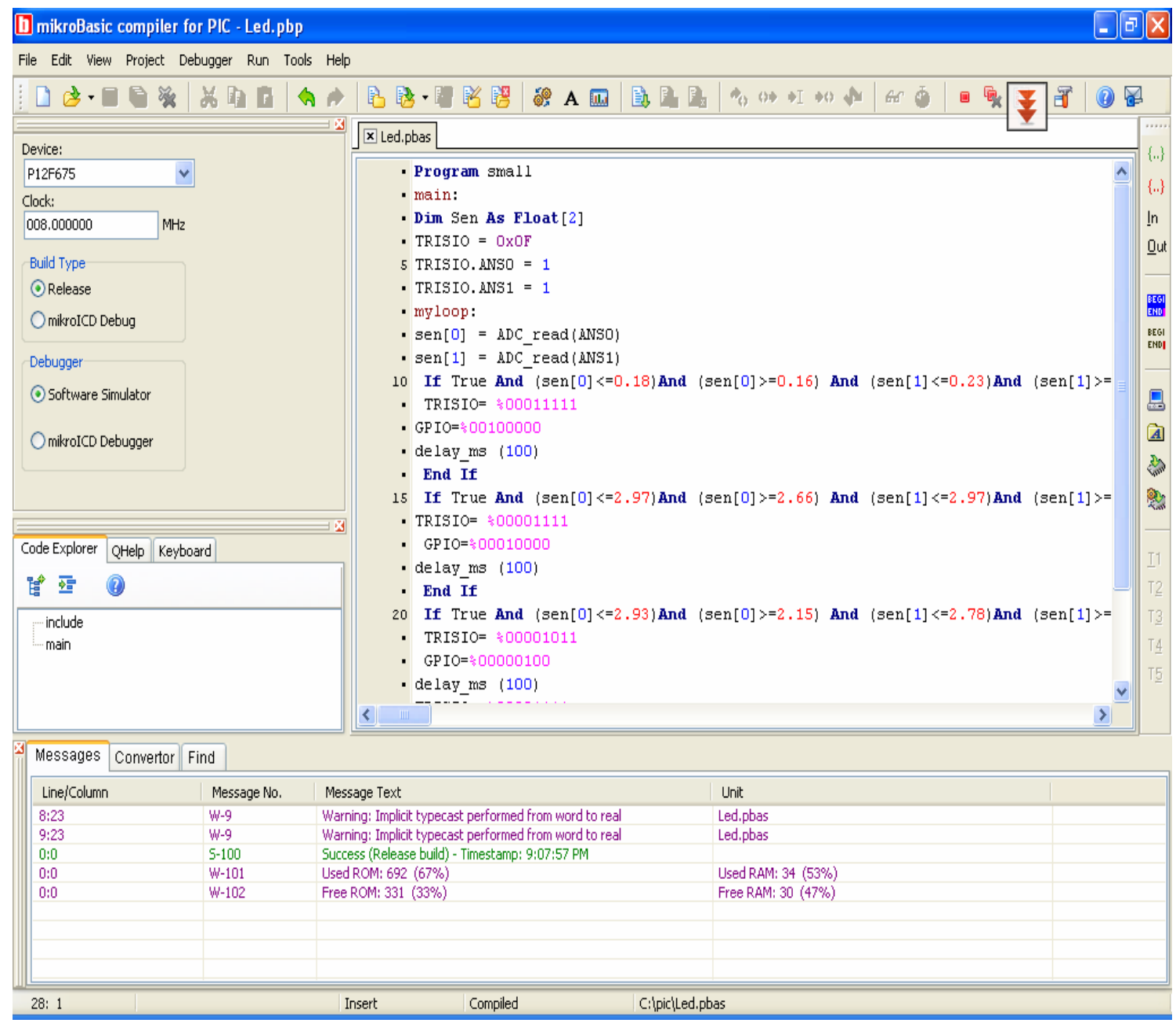

Fig.17 Editor of the MicroBasic Integrated Compiler

To optimize the program module, the parameters of number of sensors, classification criteria, and desired health categories should be chosen carefully, since these parameters will affect the usage of the memory space and the efficiency and accuracy of the performance of microcontrollers. An example of the compromise among the parameters is presented in Table 1. 
Table 1 Memory Usage of Different Applications (Variables Declared as Integer)

\begin{tabular}{|c|c|c|c|c|}
\hline $\begin{array}{c}\text { Maximum } \\
\text { Capacity }\end{array}$ & $\begin{array}{c}\text { Used } \\
\text { Flash }\end{array}$ & $\begin{array}{c}\text { Unused } \\
\text { Flash }\end{array}$ & $\begin{array}{c}\text { Used } \\
\text { RAM }\end{array}$ & $\begin{array}{c}\text { Unused } \\
\text { RAM }\end{array}$ \\
\hline $\begin{array}{c}4 \text { sensors 3 levels } \\
\text { (Boundary only) }\end{array}$ & $999(97 \%)$ & $24(3 \%)$ & $42(65 \%)$ & $22(35 \%)$ \\
\hline $\begin{array}{c}\text { 2 sensors 2 levels } \\
\text { (MaxSSE only) }\end{array}$ & $922(90 \%)$ & $101(10 \%)$ & $36(56 \%)$ & $28(44 \%)$ \\
\hline $\begin{array}{c}\text { 1 sensor 2 levels } \\
\text { (Both) }\end{array}$ & $937(91 \%)$ & $86(9 \%)$ & $30(46 \%)$ & $34(54 \%)$ \\
\hline $\begin{array}{c}\text { 2 sensors 1 level } \\
\text { (Both) }\end{array}$ & $993(97 \%)$ & $30(3 \%)$ & $34(53 \%)$ & $30(47 \%)$ \\
\hline
\end{tabular}

To further analyze the relationship between the parameters and the usage of the memories, another simulated case was considered. The training data was generated randomly between 0 and 5 assuming that the original signal was from 0 to 5 volts and they were sampled under the 8 bit $\mathrm{D} / \mathrm{A}$ conversion mode. The parameter variables were declared as byte to save the memory space. The required flash and RAM spaces are listed in Table 2. The estimated parameters of boundaries and MaxSSE for the generated data are illustrated in Fig.18. The automatically prepared MikroBasic code and compilation result are presented in Fig.19

Table 2 Memory Usage of Different Applications (Variables Declared as Byte)

\begin{tabular}{|c|c|c|c|c|}
\hline $\begin{array}{c}\text { Maximum } \\
\text { Capacity }\end{array}$ & $\begin{array}{c}\text { Used } \\
\text { Flash }\end{array}$ & $\begin{array}{c}\text { Unused } \\
\text { Flash }\end{array}$ & $\begin{array}{c}\text { Used } \\
\text { RAM }\end{array}$ & $\begin{array}{c}\text { Unused } \\
\text { RAM }\end{array}$ \\
\hline $\begin{array}{c}\text { 3 sensors 3 levels } \\
\text { (Boundary only) }\end{array}$ & $845(82 \%)$ & $178(18 \%)$ & $32(50 \%)$ & $32(50 \%)$ \\
\hline $\begin{array}{c}4 \text { sensors 4 levels } \\
\text { (MaxSSE only) }\end{array}$ & $941(91 \%)$ & $82(9 \%)$ & $28(43 \%)$ & $36(57 \%)$ \\
\hline $\begin{array}{c}\text { 2 sensors 3 levels } \\
\text { (Both) }\end{array}$ & $918(89 \%)$ & $105(11 \%)$ & $31(48 \%)$ & $33(52 \%)$ \\
\hline $\begin{array}{c}3 \text { sensors 2 levels } \\
\text { (Both) }\end{array}$ & $928(90 \%)$ & $95(10 \%)$ & $32(50 \%)$ & $30(47 \%)$ \\
\hline
\end{tabular}




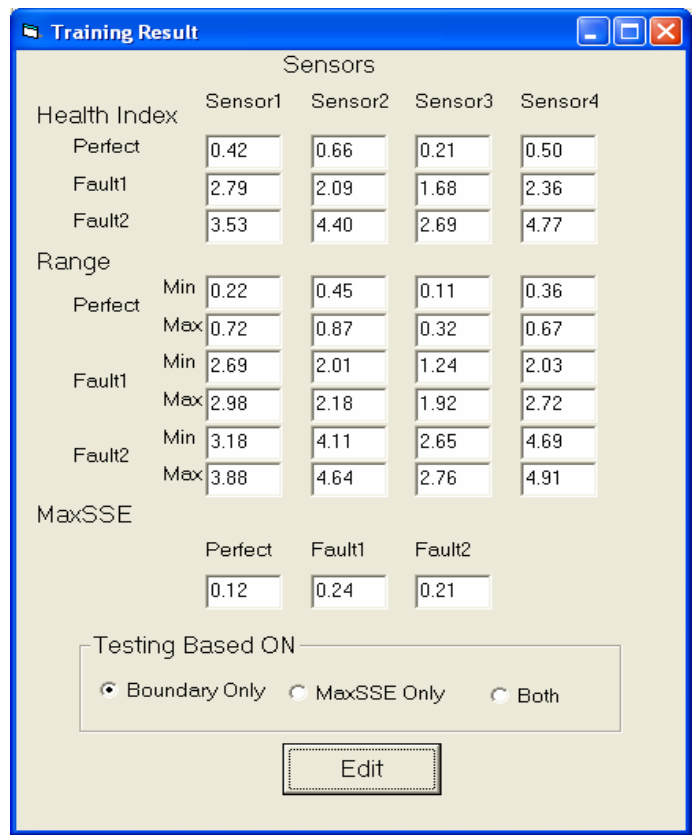

Fig.18 Boundaries of Signals from Four Sensors

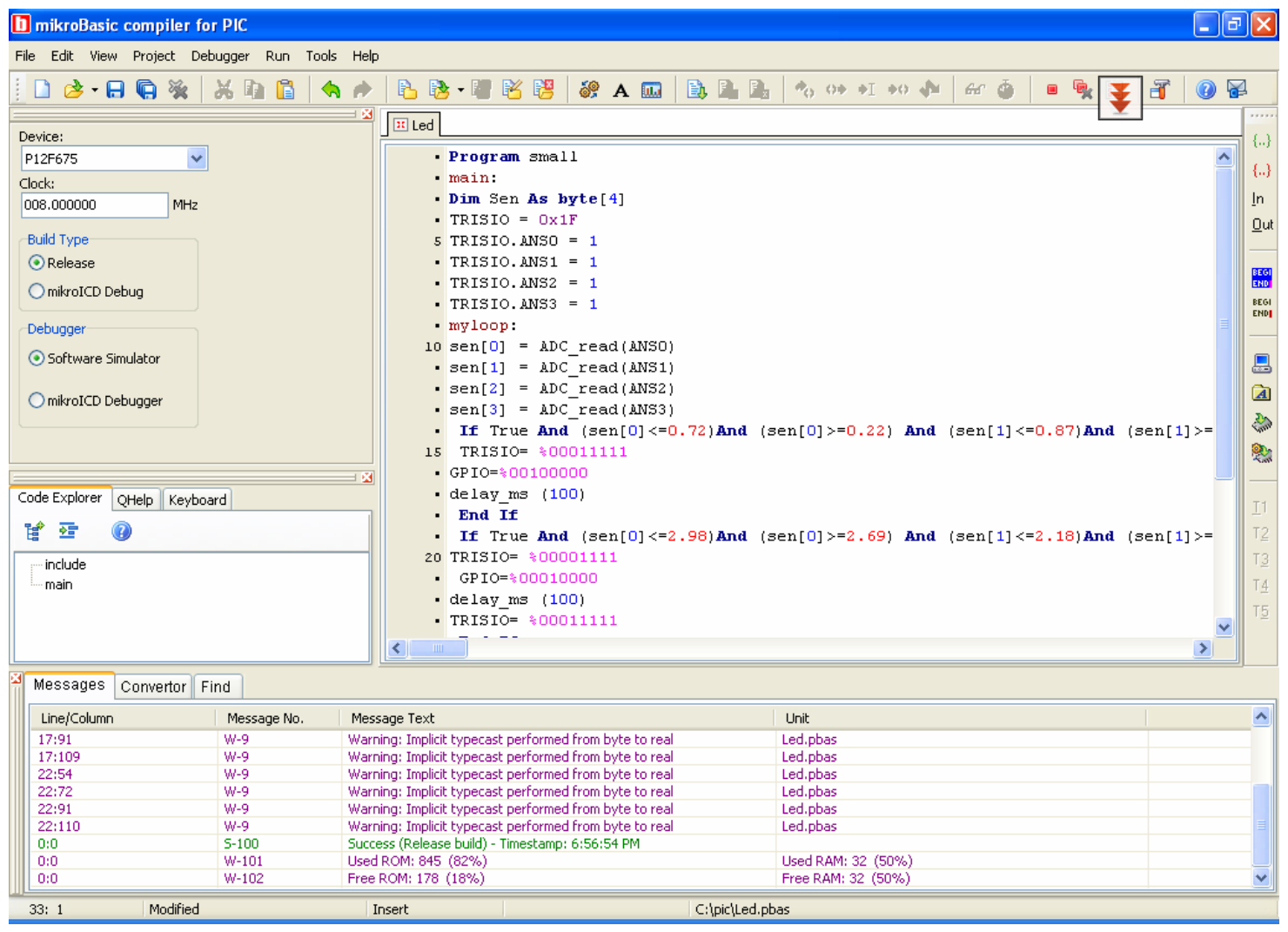

Fig.19 Compilation Results in MikroBasic 
Comparing the memory usage in Table 1 and Table 2, it indicated that calculating the sum of squared error took more memory space, and the final machine code was much more compact if the parameter variables were defined as byte instead of floating point type.

For the two simulated cases, the SCIBR correctly classified all the different categories based on boundary and/or MaxSSE methods. The automatically prepared code could be compiled in MikroElectonic's MikroBasic environment without any error. However, the memory issue should be addressed, which requires optimizing the program to fit into the memory capability of specific PIC device. The SCIBR can be conveniently implemented for many applications. However, for more complex cases other classification methods such as fuzzy logic and neural networks may be used. 


\section{CHAPTER V \\ PERFORMANCE OF IBR ON SIMULATED SYSTEMS}

To evaluate the performance of the proposed IBR approach, simulations of health monitoring for several systems were conducted. In this chapter, the modeling and simulation of a high displacement actuator, a turbojet engine, a four rotor helicopter, and their health monitoring systems using IBR were illustrated. The results of the simulations proved that IBR systems are effective and accurate in identifying the structural integrity of complex systems.

\subsection{Simulation of a High Displacement Actuator}

SpaceShipOne's space flights have demonstrated the advantages of low-cost reusable space vehicles. The recent success of UAVs in the battlefield has proven the capabilities of autonomous systems. Future space access vehicles will inherit many characteristics from the designs of SpaceShipOne and modern UAVs. They can be designed smaller, lighter and less complex than the current space shuttle. However, the mishap rate of autonomous systmes is much higher. Installing advanced health monitoring systems is a must for the safe and reliable operation of autonomous space access vehicles.

High Displacement Actuators (HDA) can be used in autonomous systems to actuate parts or sensors in a precise fashion. The movement is achieved by the deformation of the piezoelectric ceramic elements under applied electric field. To maximize the displacement of the actuators, generally, the stack or multilayer piezoelectric ceramics are developed to amplify the small actuated displacement of each thin piezoelectric sheet. The design of piezoelectric stack actuators is complicated when the electronics, material 
properties, such as drainage, and mechanical components are considered (Pozzi and King 2003).

In this simulation, the characteristics of the HDA was prepared by considering an inchworm piezomotor. An inchworm-type piezomotor is a linear piezomotor composed of 3-5 piezoelectric actuators, which simulats the motion of an inchworm. The design of inchworm piezomotors entails jerking during clamping motions, which is the source of position errors (Zhang and Zhu 1997). To optimize the control of the piezomotor and increase the accuracy of movement, the IBR was applied to monitor the temperature change and the displacement error when the piezomoter moved at a constant speed. Generally, when the controller requests the piezomoter to move to the desired position, a step input is issued to the system. The piezomoter will move to the desired location with some delay after the transition period. During this time, an IBR will send serious error codes when the difference between the input and the output is large. To avoid these misalarms, a time series model was proposed to estimate the output values. The simplified block diagram of a piezomotor and its controller are presented in Fig.20 (Tansel et al. 2007).

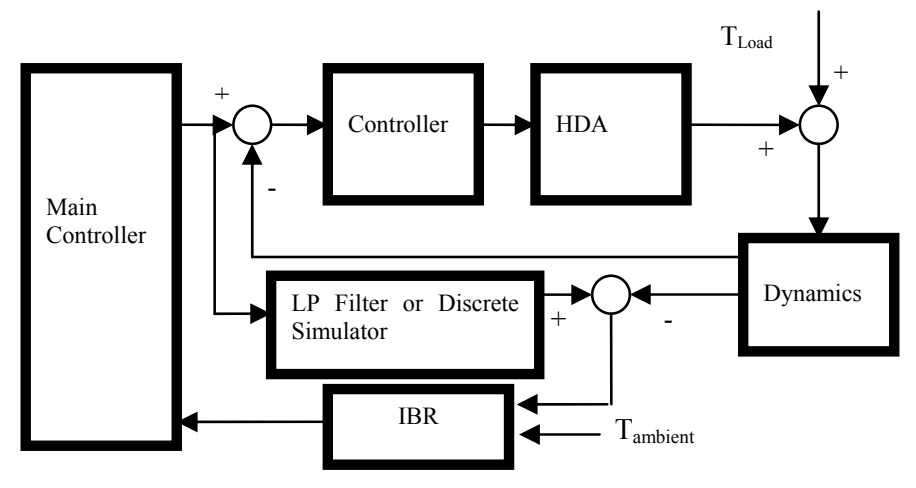

Fig.20 Diagram of a Simulated HDA and its Health Monitoring System 
In this approach, the encoder of the IBR calculated the FFT of the simulated accelerometer signal. The frequency and amplitude of the highest spike were periodically calculated and presented to the classifier of the IBR. A non-linear system was prepared to simulate the step response of the HDA by two linear transfer functions. One of the transfer functions was subjected to the input signal and moved the object with the similar rise as shown in Fig.11 a of Ref. (Mrad et al. 2003). When the HDA reached to the desired position there were a series of high frequency oscillations according to the reference. To generate the similar response, a secondary transfer function was connected in parallel and subjected to a customized input starting at 0.01 second.

To minimize the number of error codes and the severity of health levels during the transition period, a reference signal was obtained using a low-pass Chebyshev II filter designed with $900 \mathrm{~Hz}$ cutoff frequency. Thus, the difference between the reference and the displacement of the HDA was reduced. To improve the accuracy of the reference signal, a ARMAX model was used to estimate between the input and the output of the HDA using the following transfer function,

$$
G(z)=\frac{0.00057497 z+0.00057192}{z^{2}-1.949 z+0.95011}
$$

The proposed model of the HAD and its health monitoring system are presented in Fig.21. The encoder of the IBR generated a reference signal by passing the input signal of the HDA through the above equation. The classifier determined the HI value by evaluating the two input signals. The first value was the difference between the displacement and the reference signal. The second value was the ambient temperature. 
The LUT of the classifier was programmed to tolerate lower accuracy levels at the extreme operating temperatures of the satellite.

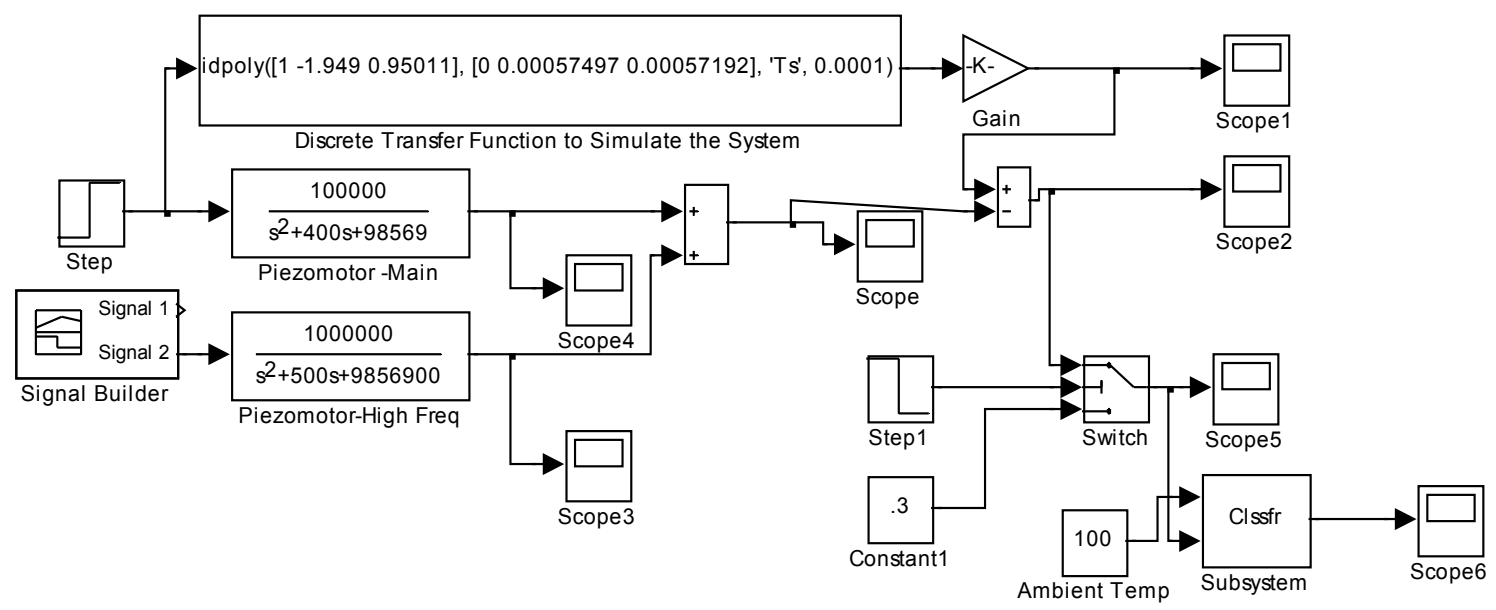

Fig.21 Simulink Model of the Piezomotor and its ARMAX Based IBR System

The HI value variations with and without the ARMAX model are presented in Fig.22 a) and b) respectively. Only a few low HI values were issued when the ARMAX model was used. These poor values could be easily corrected by programming the Index History Evaluator (IHE) not to give wrong alarms unless that value is repeated a certain number of times.

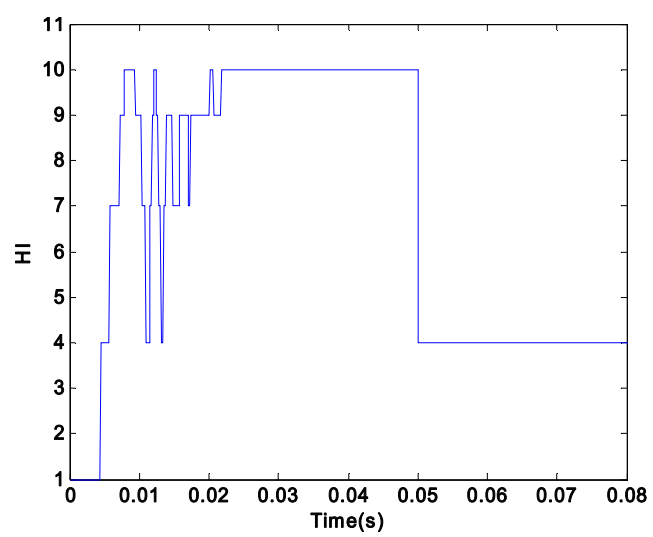

a) Without Any Encoder

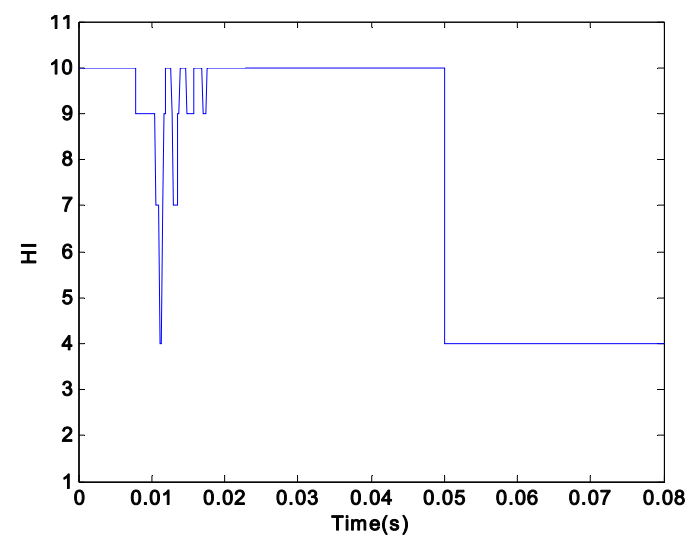

b) With ARMAX Based Encoder

Fig.22 Variation of HI Values During the Transition Period 


\subsection{Simulation of a Turbojet Engine}

The design of the high-energy, liquid-rocket engines was significantly improved in the 1944-1950 era (Osbomr et al. 1997). Today's most powerful rocket engines, which had been used for the space shuttle, were designed based on similar principles. A series of experiments that started in 1976 had improved the design of rocket engines (Biggs 1992). Recently, two approaches have been considered for future launch vehicles. Leading research laboratories, such as Marshall Space Flight Center, have been developing liquid rocket engines for small-class launch vehicles (Trinch 1994). SpaceShipOne uses a hybrid rocket design which burns hydroxy-terminated polybutadiene (HTPB), a synthetic rubber , and nitrous oxide, a liquid oxidizer (Sweetman 2004). The design is less complex since it does not need complex fuel pumps.

The engine is the most critical part of space vehicles. To improve the safety and reliability, and increase the reuse capacity of engines, the requirement of high performance condition-based monitoring of the operating health of propulsion systems is continuely growing ( $\mathrm{Wu} 2005$ ). However, the health monitoring systems of the engines of aerospace vehicles are very complex. On the other hand, acquiring operational information of rocket engines in laboratory environment is quite expensive. Since carrying out experiments on small turbojet engines are safer, easier and more affordable, more data is available comparing to rocket engines. Once a health monitoring system is developed for turbojet engines, it may be adopted to the rocket engines with relatively simple adjustments. Thus, the model of the SR-30 turbojet engine and its controller 
(Watanabe et al. 2004) was used to testify the performance of the proposed health monitoring system for an aerospace engine. In this simulation, multiple IBR nodes were applied to estimate the health conditions of the system according to the vibration and the temperature information acquired by the model based analysis. The simplified block diagram of the engine and the proposed health monitoring system is presented in Fig.23.

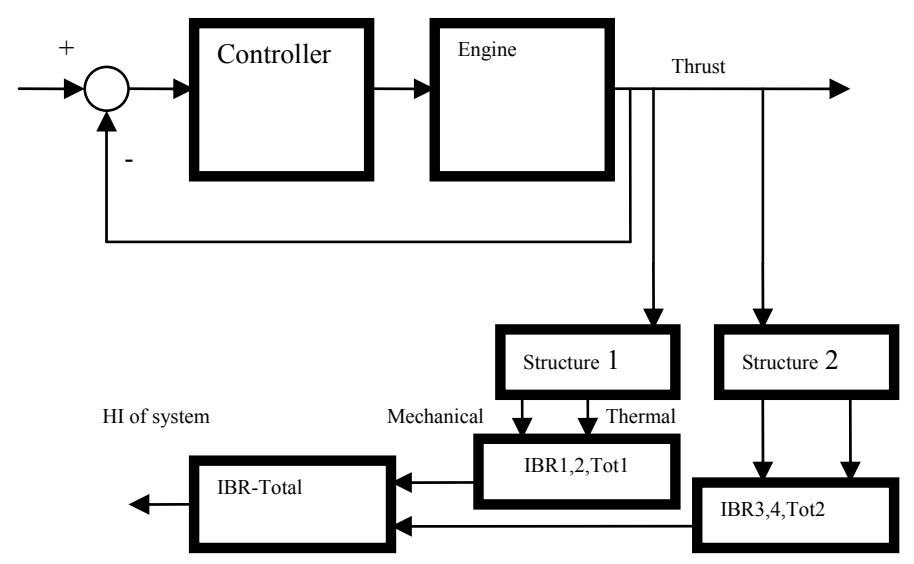

Fig.23 Diagram of a Simplified Engine with Support Structures and the Proposed SHM

The engine was assumed to be held with two identical supports. Random vibrations and the high frequency oscillation at the rotational speed were assumed to excite the supports by the engine. In addition, the temperature of the support depends on the heat exchange with the engine. The natural frequencies of the structures were assumed to be less than $100 \mathrm{~Hz}$. In accordance with these assumations, the vibration and the temperature fluctuation of the two supports were simulated. Each support was represented by a one d.o.f mass-spring-damper system. A first order transfer function was chosen to describe the heat transfer between the engine and the support. The engine thrust was assumed to excite each support with random vibrations less than $100 \mathrm{~Hz}$ and the unbalance of the 
pump would generate the $600 \mathrm{~Hz}$ harmonic vibration (considering the rocket engine). Details of the simulated engine and the proposed health monitoring system are presented in Fig.24

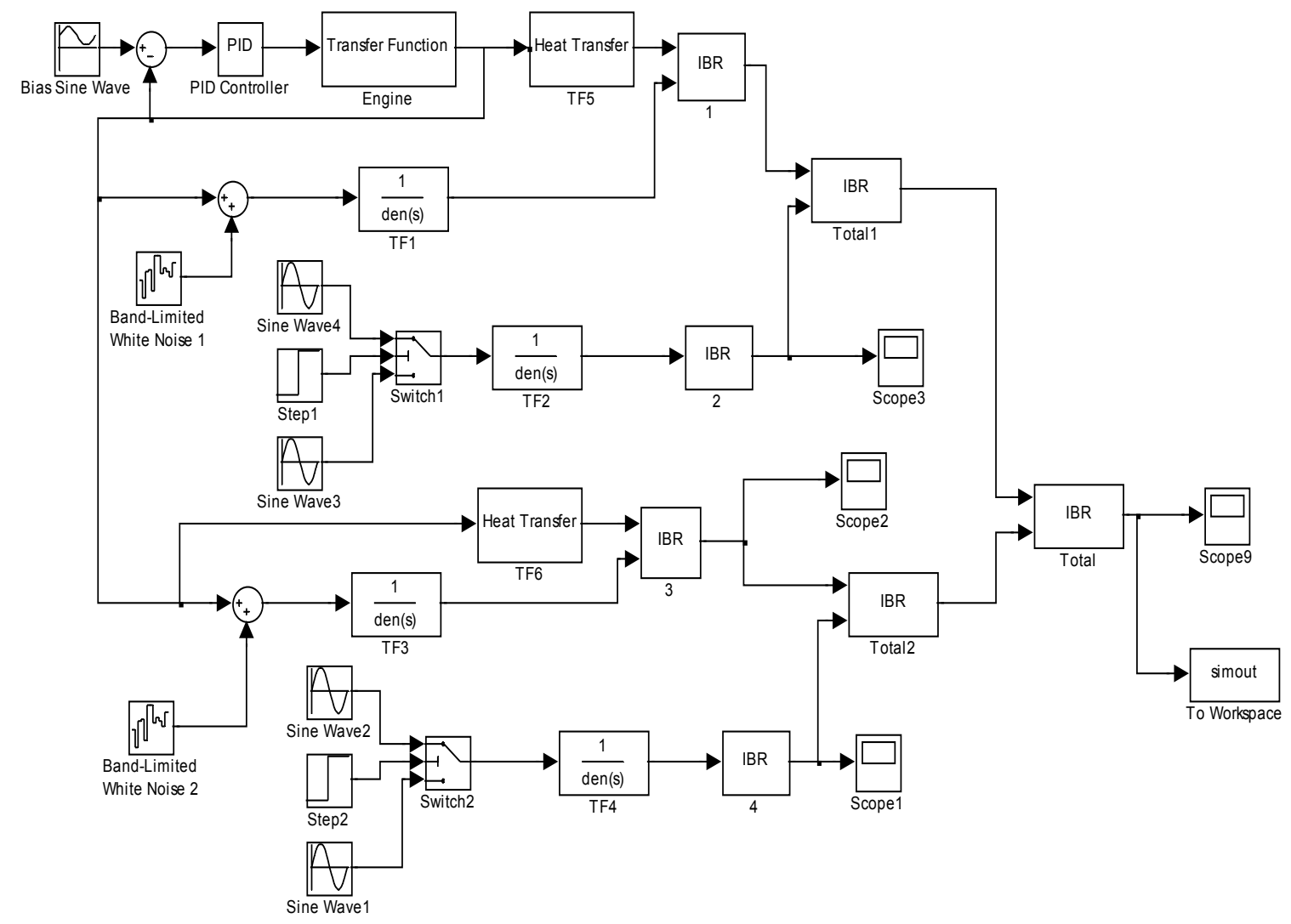

Fig.24 Simulink Model of the Engine and the Proposed Health Monitoring System

Two identical IBRs were used to evaluate the health conditions of two simulated structural elements by considering the operating temperature and either the dominant frequency or the amplitude level of the spectrum of the vibration. For each support, three different levels of IBRs were utilized. IBRs of the lowest level evaluated certain aspect of the local health condition. One IBR, which is illustrated in Fig.25 a), evaluated the low frequency vibrations and the temperature. Another IBR evaluated the high frequency vibrations, which is shown in Fig.25 b). To interpret the simulated accelerometer signals, 
the encoder calculated the according FFT. For low frequency vibrations, only the value of the dominant frequency was sent to the classifier to detect any structural weakness. For high frequency vibrations, only the highest amplitude in 400-600 Hz range was inspected to identify any unbalance or future failure. The HI estimations of the two IBRs at the lowest level were sent to the IBRs at the second level to determine the condition of the support. The HI values of the two supports were then sent to the IBRs of the highest level to determine the condition of the complete system.

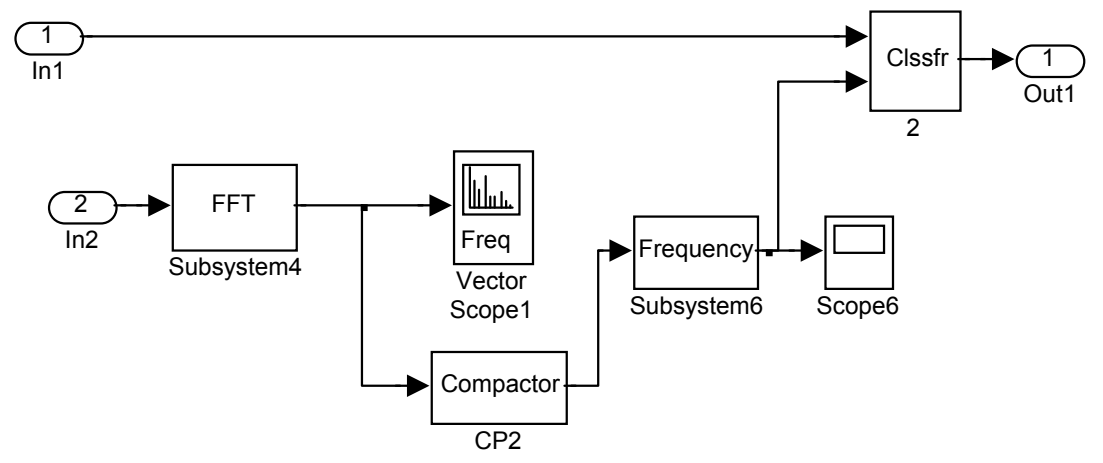

a) Block Diagram of IBR1 and IBR3.

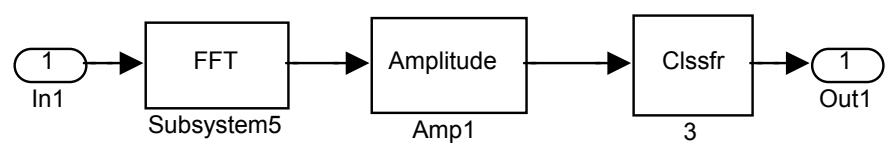

b) Block Diagram of IBR2 and IBR4.

Fig.25 Diagrams of IBR Nodes for the Simulated Engine

The estimation of the system health conditions is presented in Fig.26. When the reference signal, the input of the system, was raised from " 0 " to a harmonic signal varying around "1", the engine was in a transition stage to warm up. The HI of the highest level IBR, which indicated the condition of the overall system, fluctuated and 
reached to "10". At 15 th second, the simulated unbalance occurred and the amplitude of the high frequency harmonic excitation was drastically increased. Then the IBR immediately identified it and the HI value fell to "2" indicating a serious problem.

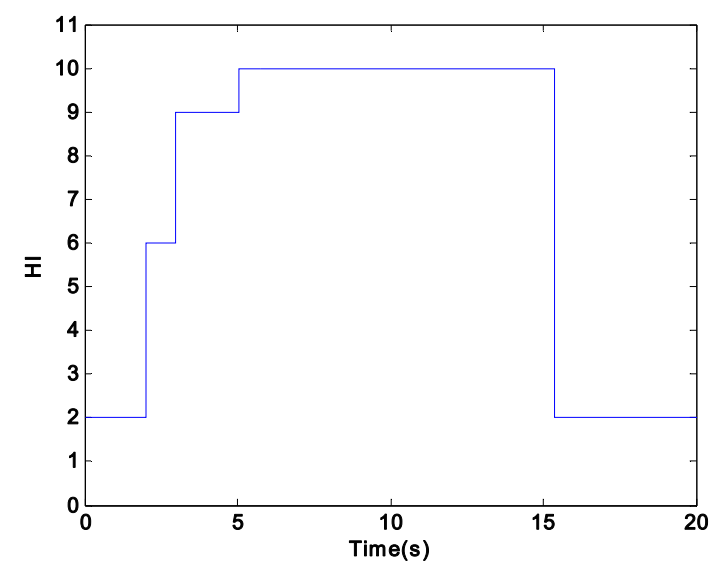

Fig.26 HI Values from Warming Up to Bearing Failure

\subsection{Simulation of a Four Rotor Helicopter}

During the last several decades, four rotor helicopters have attracted much research interest due to their capability of quasi-stationary flight as well as vertical take-off and landing. They are underaccurate dynamic vehicles rotating two pairs of fixed pitch angle blades to obtain the motion behaviors. The quad-rotor helicopter is controlled by varying the angular speed of each rotor, and the main thrust is the sum of the thrusts of all the motors. While it is working, the front and rear rotors rotate counterclockwise and the left and right rotors rotate clockwise. This challenges the control strategy of the system since movements of the helicopter are highly coupled with each other. Several researches have been carried out to optimize the control of four rotor helicopters. Castillo et al. (2004) proposed a controller based on Lyapunov analysis using a nested saturation algorithm. Li et al. (2006) adopted time-optimal control by using genetic algorithm. Altug et al. (2005) 
and Suter et al. (2002) used the pictures of a visual camera to estimate and control the position and orientation of the helicopter.

In terms of flight guidance of UAV, the control systems usually follow the programmed waypoints to reach to their targets. However, as the flight condition, structural integrity, and other factors may vary during the mission, it is necessary that the Autonomous Guidance and Control (AG\&G) system adjust its parameters with the information of integrated SHM to maximize the performance of UAVs. The proposed architecture (Bckriaj 2006) of the integrated system is shown in Fig.27. The controller processes the gathered information of flight path, flight condition, and structural health conditions simultaneously, and then determines the proper control characteristics. If necessary, abnormalities may be reported to the ground station.

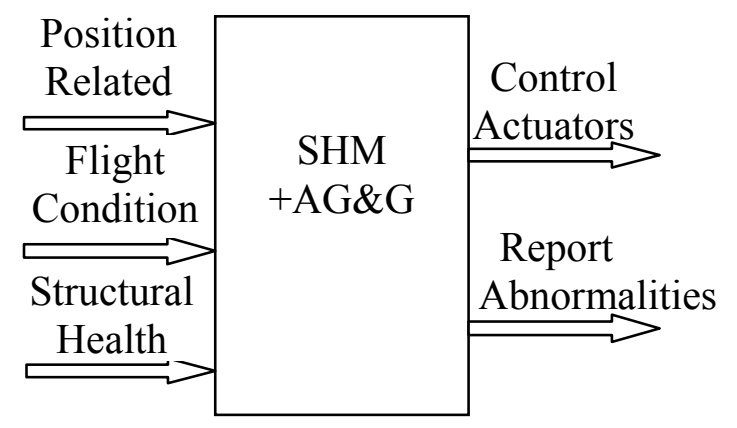

Fig.27 Integrated System of SHM and AG\&G

The small helicopter has four rotors and according subsystems, and these structures are assembled from low-cost components. Thus, the possibility of component failure may increase. The possible faults of the small four rotor helicopter will be classified by IBR with a HI. Based on the health condition, the controller will reconfigure its parameters to maintain the stable and acceptable performance of the system. One advantage of this 
rotary wing unmanned aerial vehicle is that it can achieve quasi-stationary hovering, so that it can stay above targets for surveillance and inspection applications. Thus, for these kinds of applications, it is quite important for the controller to get it to the desired altitude and keep it there robustly and stably. In this study, PID control and Fuzzy Logic control integrated with IBR were investigated through simulations to guide this helicopter to achieve its target under different health conditions. The simulated helicopter model was the Draganflyer manufactured by RCToys.

\subsubsection{PID Control}

PID control is one of the most commonly used feedback control in industry. It includes proportional, integral, and derivative elements which address present, past and future errors. The algorithm of PID controller can be written as:

$$
u(t)=K_{p}\left(e(t)+\frac{1}{T_{i}} \int_{0}^{t} e(\tau) d \tau+T_{d} \frac{d e(t)}{d t}\right)
$$

Where, $u(t)$ is the control signal, $e(t)$ is the control error, and $K_{p}, T_{i}, T_{d}$ are proportional gain, integral time and derivative time respectively. For specific applications, part of PID controls may be used, such as PI control or PD control.

To design a PID controller, first, the action mode should be decided, and then the parameters should be adjusted in order to achieve the control target appropriately (Araki and Taguchi 2003). In accordance with the dynamic model of the four rotor helicopter, a PID controller with all the proportional, integral, and derivative elements were developed. A trial and test process was carried out to determine the parameters of $K_{p}, T_{i}$, and $T_{d}$. In our case, since we only considered controlling the helicopter to move along vertical direction, after decided $T_{i}$ and $T_{d}$, only $K_{p}$ was adjusted for different control purposes. The 
Simulink model (Querry, 2006) of the whole system is presented in Fig.28 a), and

Fig.28 b) shows the details of the PID controller and the SHM system using IBR to control the helicopter through changing the voltage allocated to rotors.

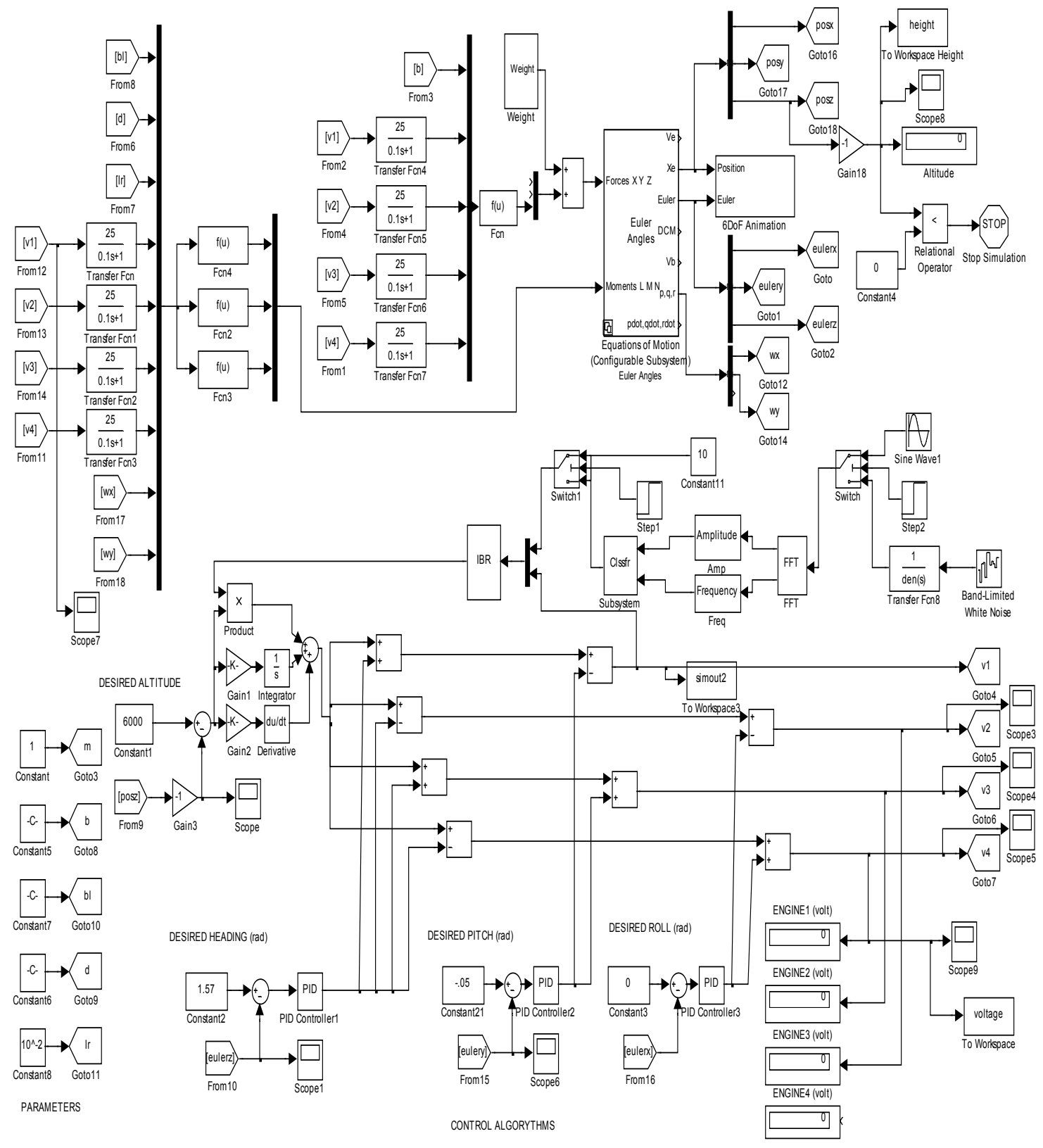

a) Four Rotor Helicopter and its Health Monitoring System

Fig.28 Simulink Model of the Four Router Helicopter System 


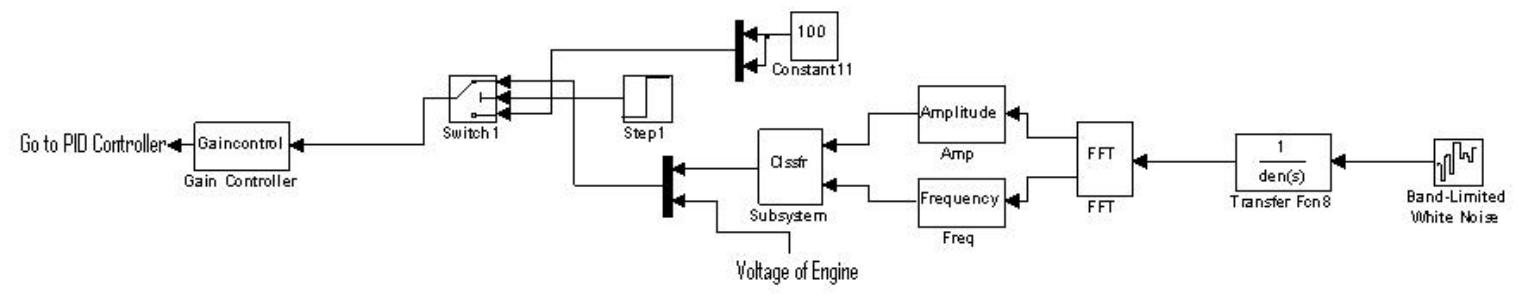

b) Details of the Health Monitoring System

Fig.29 shows an example of the simulation result of the altitude trajectory. Here the gain of the PID control is 0.022 . From the graph, the four rotor helicopter got to the desired altitude $(1500 \mathrm{~mm})$ quickly and stayed at that vertical position robustly.

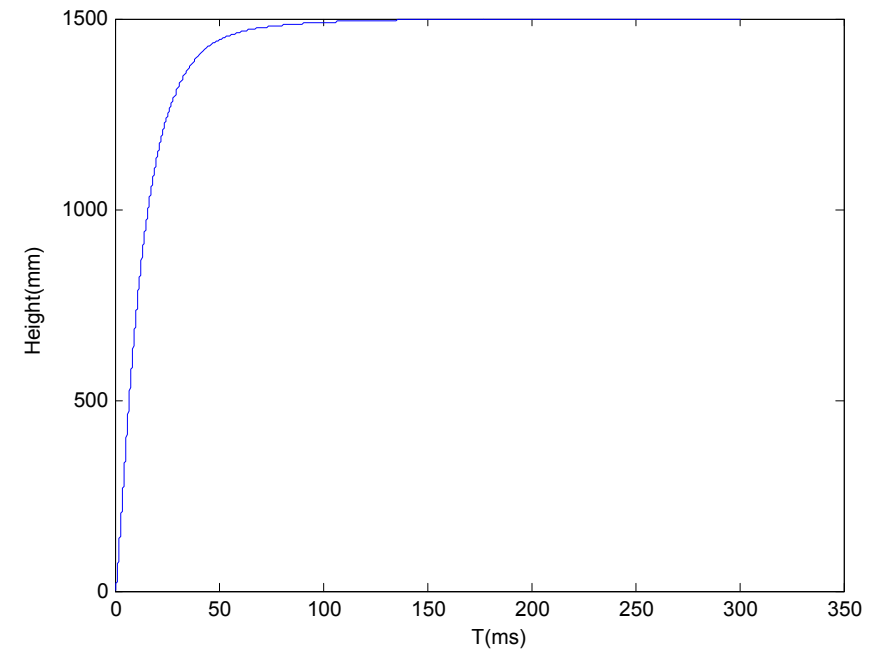

Fig.29 Trajectory of the Simulated Four Rotor Helicopter (DA:1500mm, Gain: 0.022)

But if the gain of 0.022 was used for other cases, the desired altitude could not be achieved, for example Fig.30 a) shows the simulation result when the desired altitude was $6000 \mathrm{~mm}$. The plane climbed up but then went down, it was obvious that the parameters of PID controller needed to be optimized. Thus, a trial and test process was carried out and the gain of 0.015 was found to be the best value. Fig. $30 \mathrm{~b}$ ) shows the trajectory of the helicopter when the PID controller adopted this optimized value. The same thing 
happened to other cases; certain value of the gain only worked well for a range of altitude. To obtain reliable performance, the PID controller had to reconfigure the value of the gain automatically according to different flight conditions.

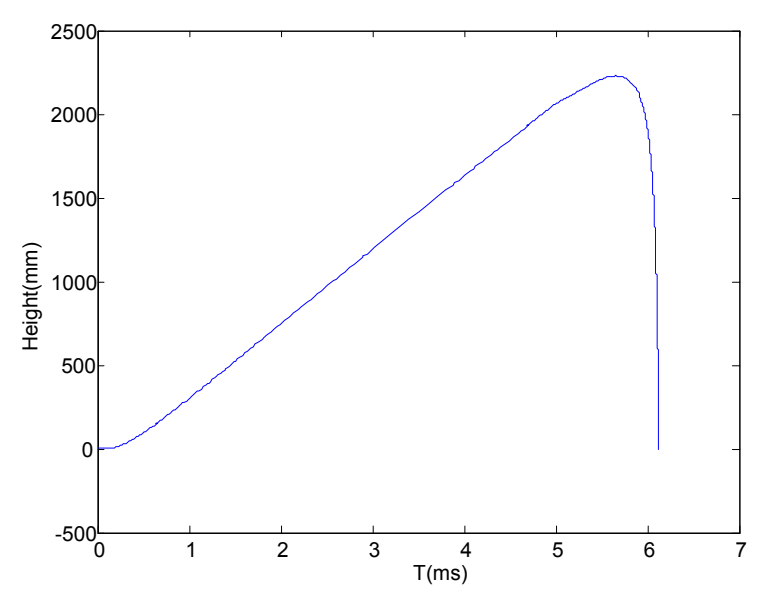

a) Gain $=0.022$

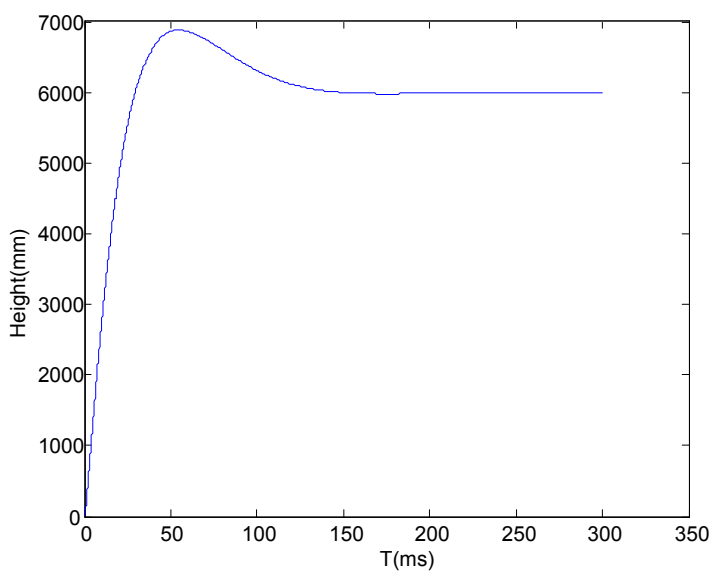

b) Gain $=0.015$

Fig.30 Trajectories with Different Gains (DA: 6000mm)

On the other hand, when the four rotor UAV doesn't work well, the voltage of the rotors will change greatly. So, through monitoring the voltage of the rotors, the health condition of the UAV could be estimated. Since in the simulation, other structural problems, such as damages of propellers, can also reflect in variation of the voltage of engines; we used the voltage of rotors as the main criteria indicating the structural integrity of the system. The SHM system used IBR to identify and classify the system's conditions according to the change of voltage, and report it to the controller to automatically adjust its parameters.

A S-function block integrating the IBR into the Simulink model was programmed. It could collect the signal of the engine voltage and the dynamic frequency responses of the tube of the four rotor helicopter's main structure. Based on the aforementioned 
information, the IBR could identify the heath index of the system and then optimize the control strategy automatically. The S-function block also can be easily upgraded to three or more dimensions of input from the sensors of the health monitoring system.

The trajectories of the UAV achieving the target of $3000 \mathrm{~mm}$ altitude under different health conditions are presented in Fig.31. The selection of the gains as $0.022,0.015$ and 0.009 was based on the health conditions of 10,5 and 2 . The desired altitude determined the initial voltage allocated on the rotors. The gain selector determined the gain of the PID controller according to the initial voltage and the HI value given by the IBR. Smaller gains were selected if the HI value is less than 10. Selection of lower gains reduced the maximum voltage applied to the electric motor, maximum thrust generated by the motor and the payload carried by the main frame.

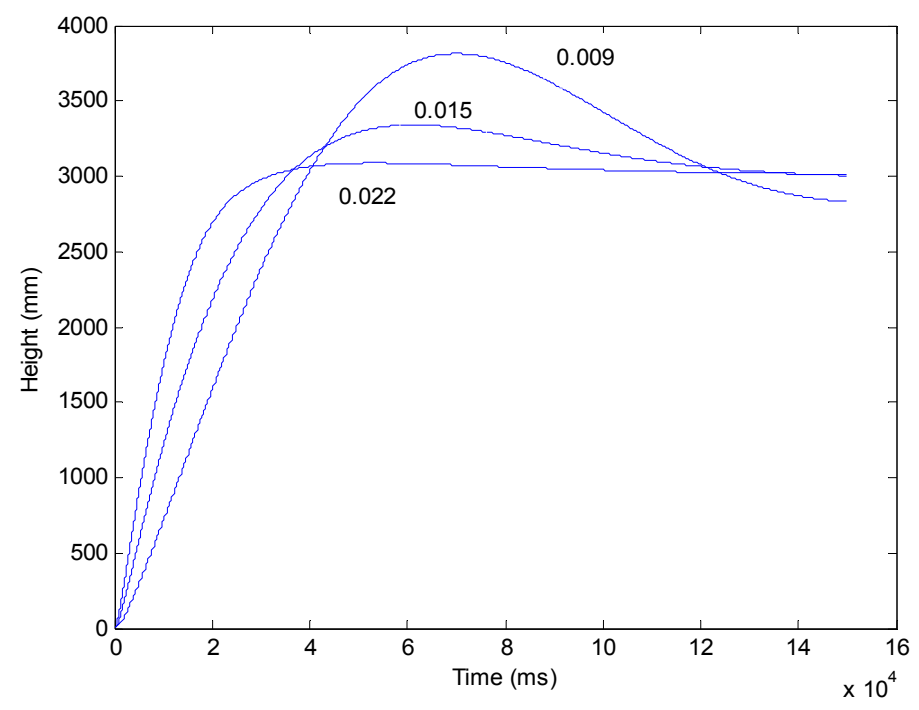

Fig.31 Trajectories under Different Conditions

The simulated studies demonstrated that the IBR is easy to program under the develop environment of Simulink. IBR could detect the simulated faults effectively and 
accurately. With the awareness of health conditions of the system, the control parameters could be optimized to gurantee the safe, robust and effective operation.

\subsubsection{Fuzzy Logic Control}

In this simulation, Fuzzy Logic control was investigated to guide the UAV to perform climbing and hovering under various conditions. As the previous simulation, IBR evaluated the flight condition and the structural integrity, and then determined the health condition of the whole system with a HI. The HI was then carried to AG\&G system. Fuzzy logic controller reconfigured its parameters based on the health condition of the system to provide fault-tolerant control, and the parameters of fuzzy logic controller were regulated by a set of rules. The implementation of the proposed SHM +AG\&G approach is presented with Simulink model in Fig. 32 .

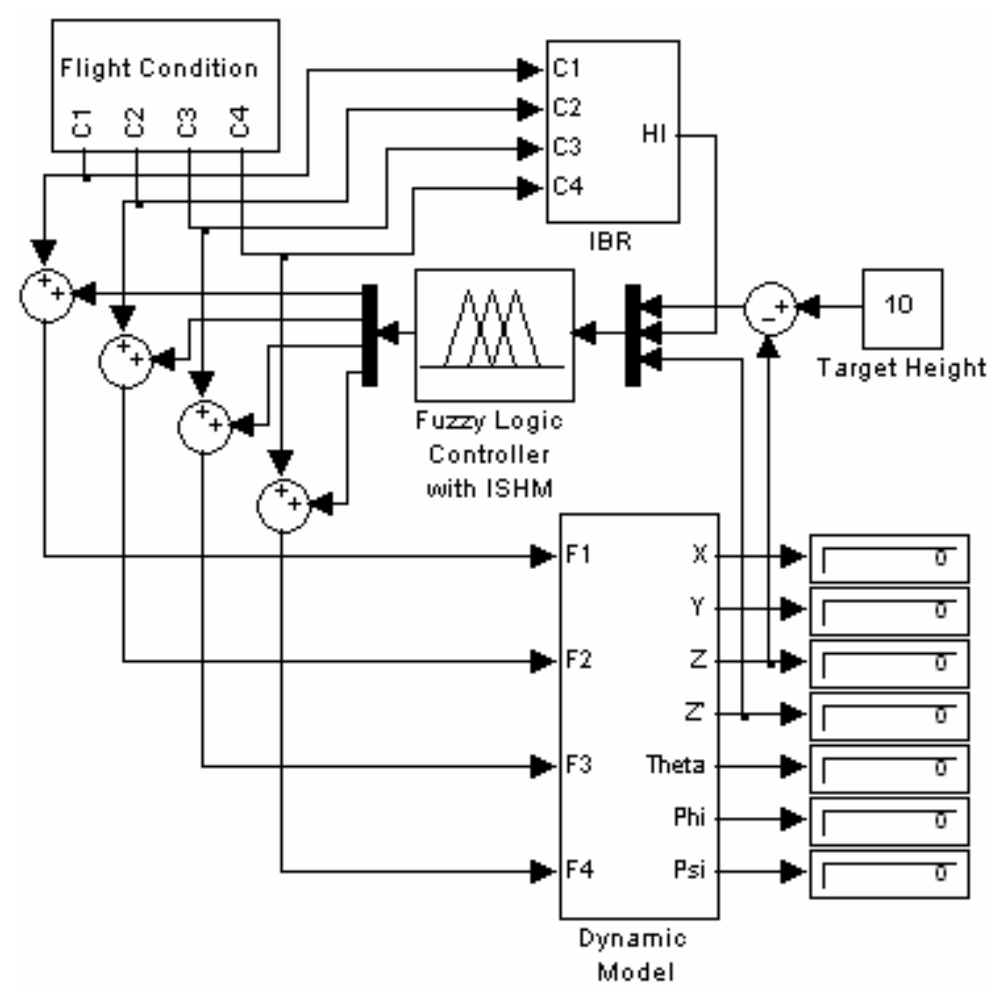

Fig.32 Simulink Model of Fuzzy Logic Control 
Since the performance of rotors was the main concern of the system health, IBR evaluated the condition of the rotors and classified it with a labeled HI, and then presented it to the fuzzy logic controller as an input. In this study, the UAV was controlled to reach the desired altitude and hover around. Fuzzy logic controller would reconfigure the control surface to reach to the target under different health conditions. The simulated fault occurred in this simulation was one or two rotors failed during the mission.

The input variables of fuzzy logic controller were the offset distance of target altitude, the vertical speed and the HI. The membership functions of offset height (z) and vertical speed (zdot) were triangular. Membership functions of HIs were trapezoidal. Fig.33 and Fig.34 illustrate membership functions of the offset and the speed respectively. Thus, according to the difference of the coordinate and the speed of the helicopter, the controller was designed to drive the offset to zero. At the same time, if the health condition changed as one or two rotors fail, the controller would reconfigure the control characteristics of rotors.

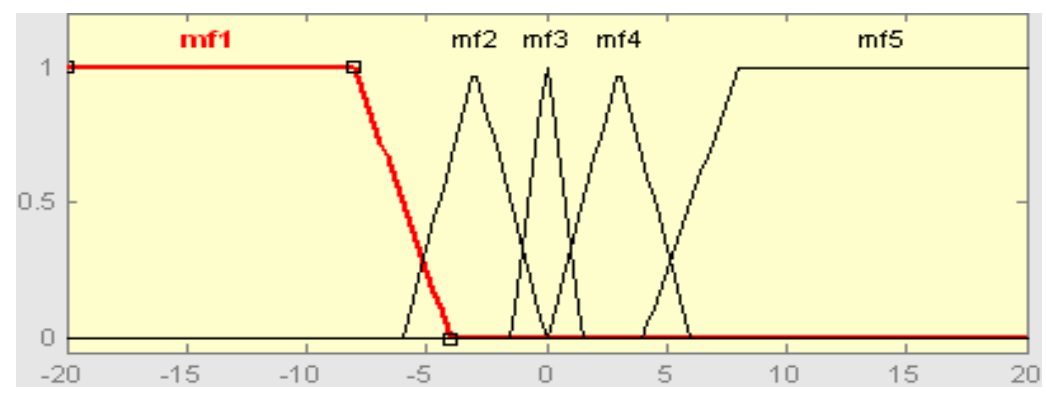

Fig.33 Membership Function of Height Offset 


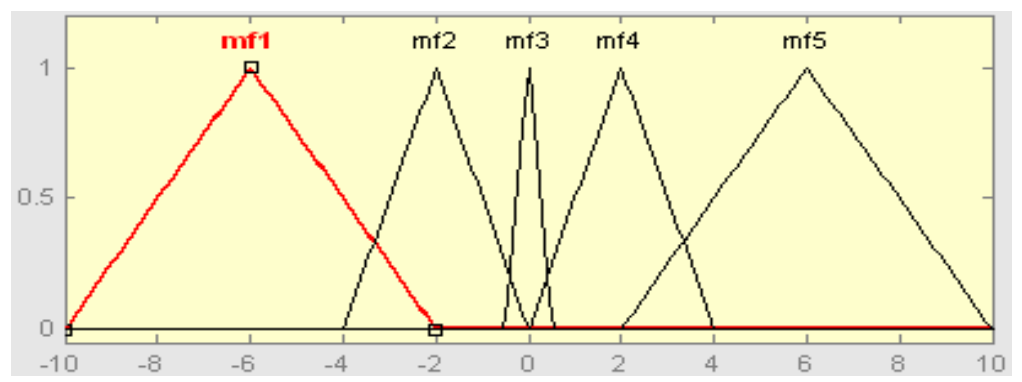

Fig.34 Membership Function of Vertical Speed

The output of fuzzy logic controller was the lift force of each rotor. The lift force is given by,

$$
f=-b w e_{1}
$$

Where, $w_{i}$ is the rotating speed of rotor $i$, which can vary with applied voltage. Membership functions of output forces are presented in Fig.35. The set of rules are shown in Fig.36. Both membership functions and rules were determined based on the prior knowledge, and they needed fine tune to make the control parameters converge.

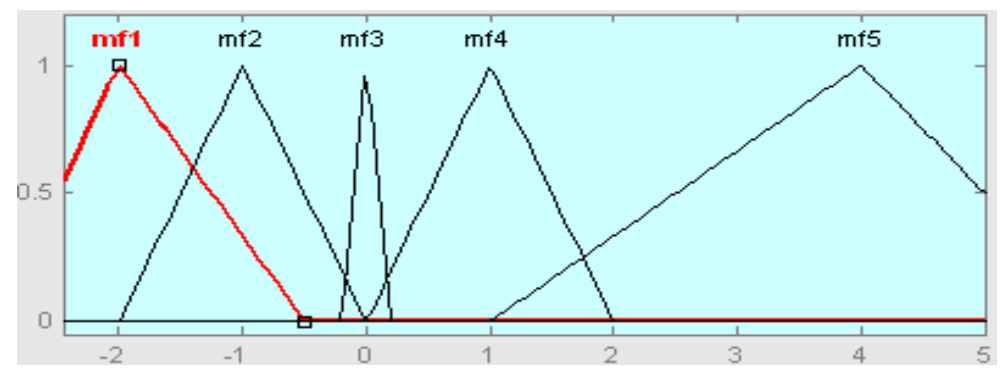

Fig.35 Membership Function of Output Force 


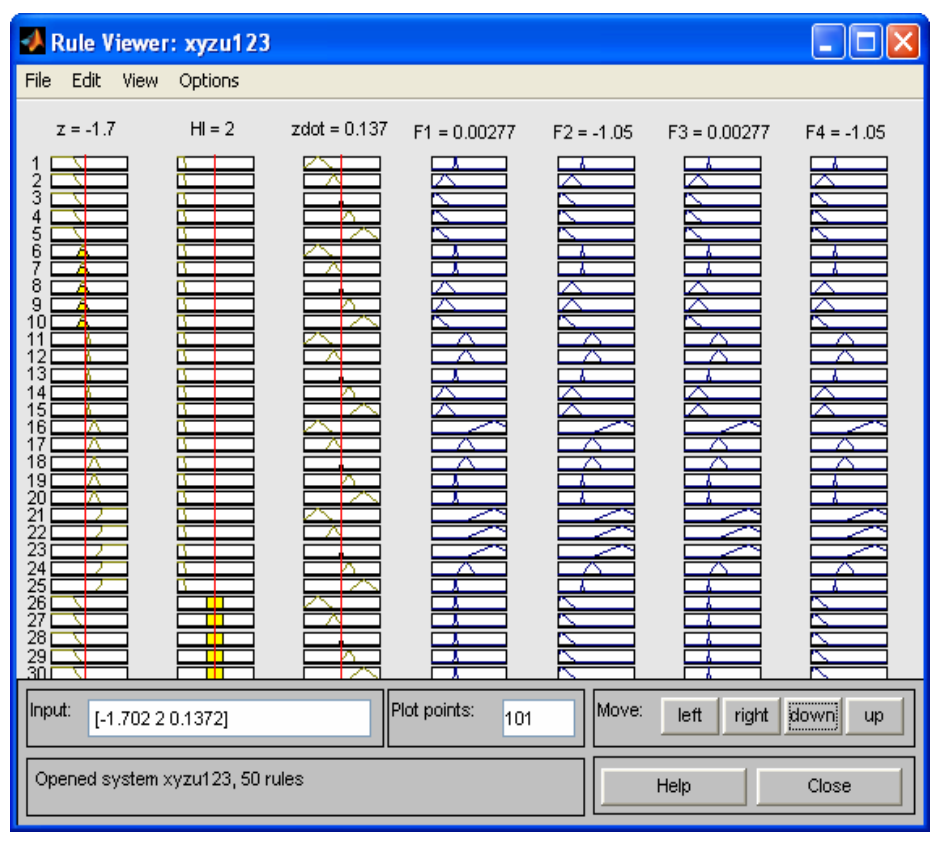

Fig.36 Rule Viewer

Fig.37 shows an example of the trajectory of the helicopter reaching to the target height from the origin. It is assumed that the health condition was perfect. As indicated from the graph, the helicopter climbed to the target under fuzzy logic controller, after a quick overshoot, it converged at the target height.

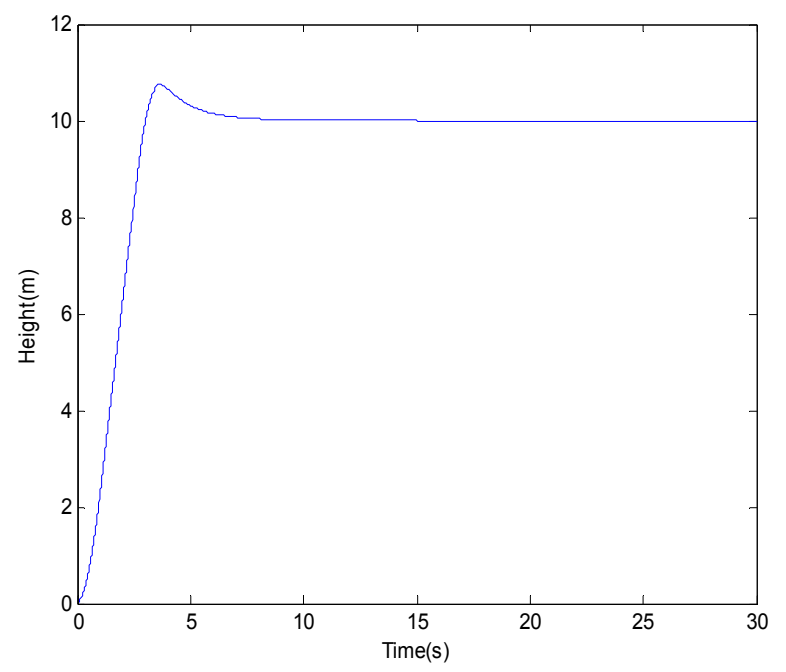

Fig.37 Simulink Result under Perfect Condition 
The fuzzy logic controller also can provide fault accommodation when components fail. Fig. 38 shows the result when simulated fault occurred. One rotor failed at 8 th second. After IBR identified this, it presented HI as 2 to the controller. Based on the rules of fuzzy logic, the controller reconfigured the output voltage applied for each rotor to continue the mission. From the diagram, the helicopter dropped first after the rotor failed. After the reconfiguration, the helicopter climbed again and reached to the target.

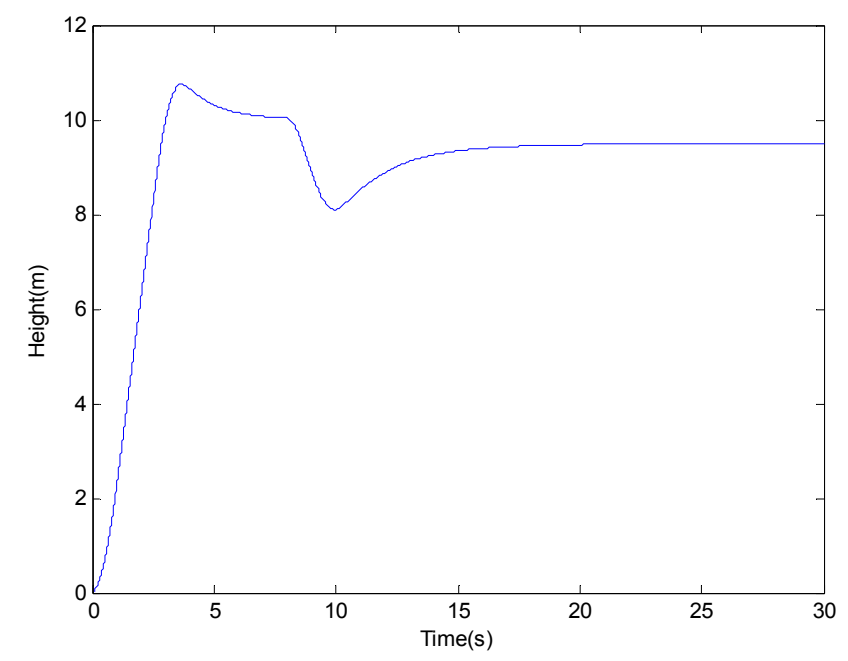

Fig.38 Simulink Result When One Rotor Failed at 8s

\subsection{Simulation on FEM Estimation of Carbon Fiber Tubes}

The objective of this part was to investigate how accurately health monitoring systems using IBR could estimate the structural integrity of a system without enough prior knowledge. SHM systems solve pattern recognition problems to identify undamaged and damaged components. However, information of damaged components and variation of damages, such as location and severity, is not 
largely provided for most of the real applications. In this simulation, a self-learning approach was integrated into IBR to implement SHM with limited information. To evaluate the proposed method, the health conditions of size and location of the damages on a carbon fiber tube of the four rotor helicopter were estimated. The Finite

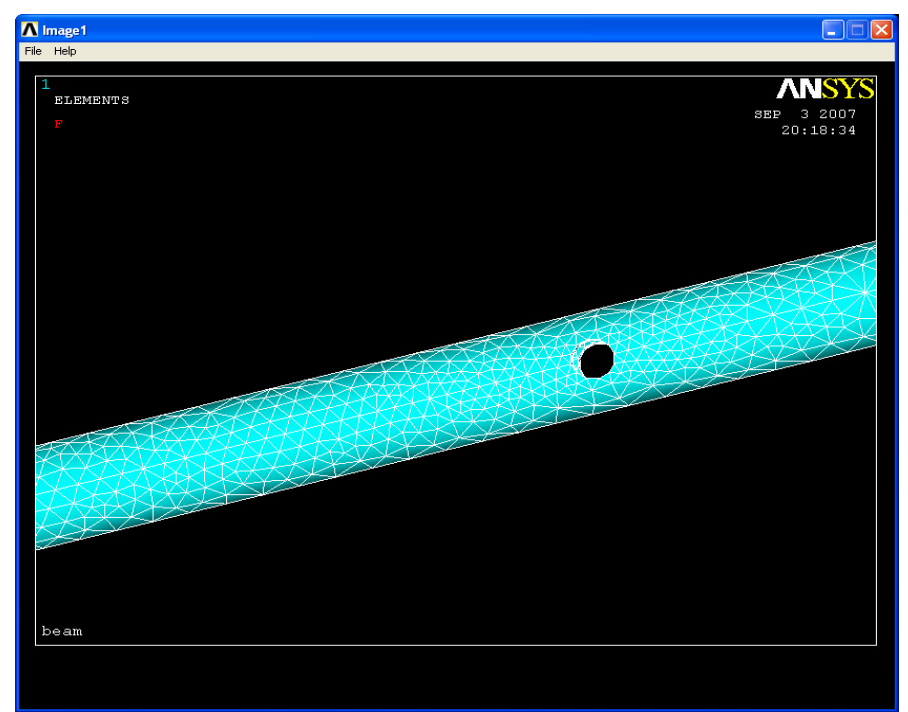

Element Method (FEM) was used to model the composite tubes and extract the dominant frequencies of the second and the third modes, which composed the data set for training and test. A series of tubes with a hole of various sizes and locations were modeled in ANSYS environment, which is presented in Fig.39.

Fig.39 FEM Model of the Composite Tube in Ansys

The affect of damage location on the variation of natural frequencies was assumed to have a general trend, as the distance from the hole to the fixed end increased, the natural frequencies would decrease. As long as the vibration signals of the tubes followed these 
trends we could develop programs to locate the damage, which would work with less sensory input and minimal training cases. In the program, several possible trends of consistent fashion were tested to fit the limited given training cases. The best fitting would then be chosen to evaluate testing data.

The developed program worked with two inputs which were the second and third mode frequencies. To testify the requirement of the proposed approach, only three cases were presented to IBR for training. The three cases were the frequencies corresponding to the damages at the fix end, at the middle, and at the free end of the tube. The program modeled the given training data with Linear, Exponential, Logarithmic and Power Law equations to estimate the trend of the damage location. Once the best fitting model was identified, the program was ready to estimate other damage locations of the structure after inspecting the encoded values from the sensors.

To test the approach, the rest available simulation data of different damage locations was used. The second and the third dominant frequencies estimated by ANSYS, which were corresponding to different damage locations on a tube, were the two inputs of the program. The one output was the estimated damage location. The FEM simulation data are presented in Table 3.

Table 3 Ansys FEM Simulation Dataset

\begin{tabular}{|c|c|c|}
\hline $\begin{array}{c}\text { Second } \\
\text { Freq }\end{array}$ & $\begin{array}{c}\text { Third } \\
\text { Freq }\end{array}$ & $\begin{array}{c}\text { Damage } \\
\text { Location }\end{array}$ \\
\hline 228.9 & 249.58 & 20 \\
\hline 233.75 & 250.13 & 40 \\
\hline 239.6 & 250.63 & 60 \\
\hline 244.75 & 251.19 & 80 \\
\hline
\end{tabular}




\begin{tabular}{|l|l|l|}
248.29 & 251.74 & 100 \\
\hline 250.55 & 252.05 & 120 \\
\hline 252.24 & 252.71 & 140 \\
\hline 253.35 & 253.42 & 160 \\
\hline 254.17 & 254.21 & 180 \\
\hline
\end{tabular}

The frequencies of the second and the third modes of the three training cases were those corresponding to the damage of $20 \mathrm{~mm}, 100 \mathrm{~mm}$ and $180 \mathrm{~mm}$ away from the fixed end of the tube. The program fitted the linear, exponential, logarithmic and power law models and presented the match on the user interface, which is demonstrated in Fig.40.

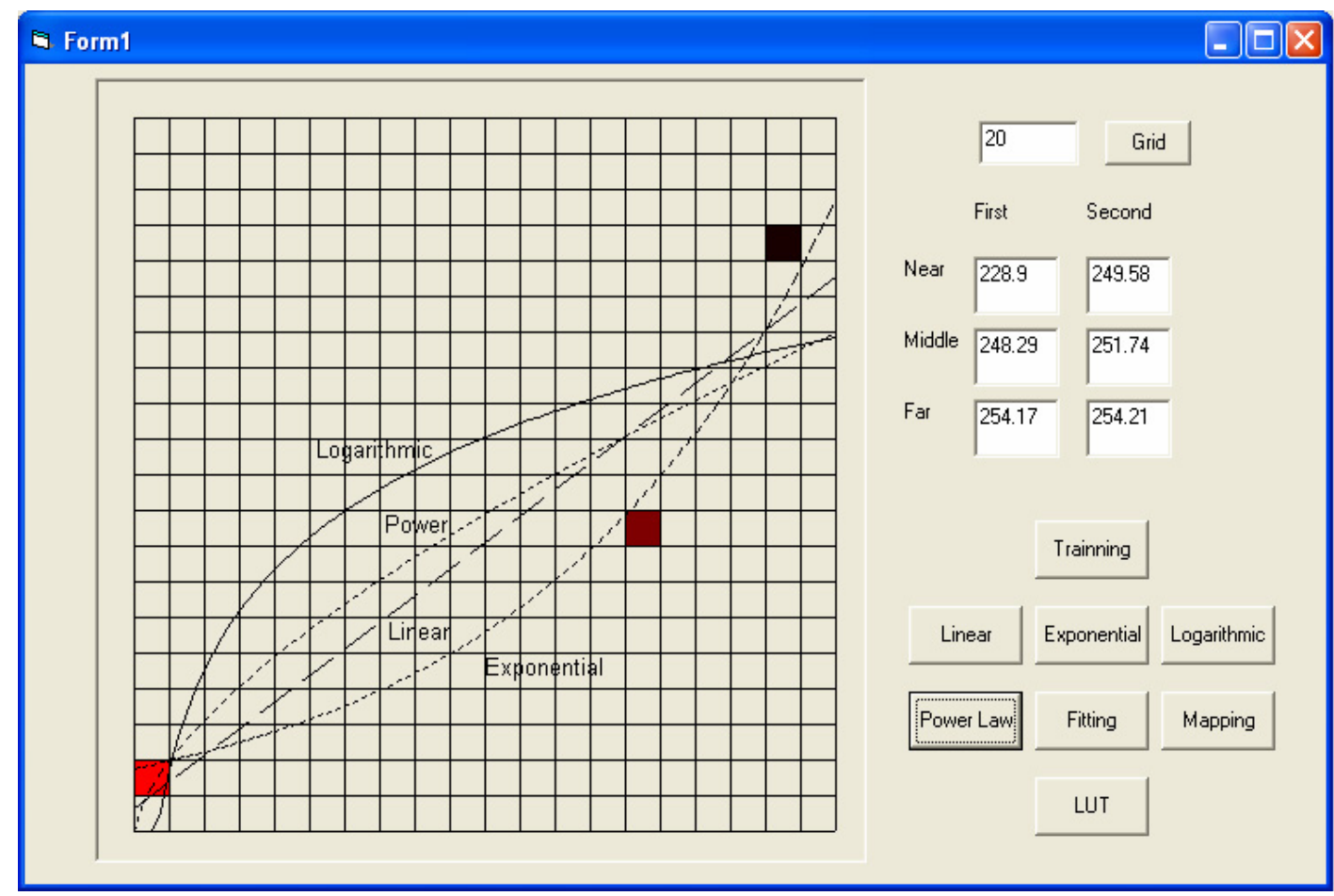

Fig.40 Fitness of Linear, Exponential, Logarithmic and Power Law Models Matching the Trend of the Simulation Data

After the best matching trend was identified, the self-learning approach mapped the segments of the curve and the area around the curve. The inspiration of this approach came from the well known Restricted Coulomb Energy (RCE) type neural networks. 
RCE networks learn the training cases by creating a new hidden node when they cannot classify the output of a given case correctly. Each hidden node basically refers to a space. If the encoded values of a test case is within the assigned space of a hidden node it will be classified as the same category which the hidden node is prepared for during the training. Similarly, we assigned values to the spaces, which surrounded the identified trend curve. Fig.41 shows the example of the exponential fitting curve and the estimated parameter mapping.

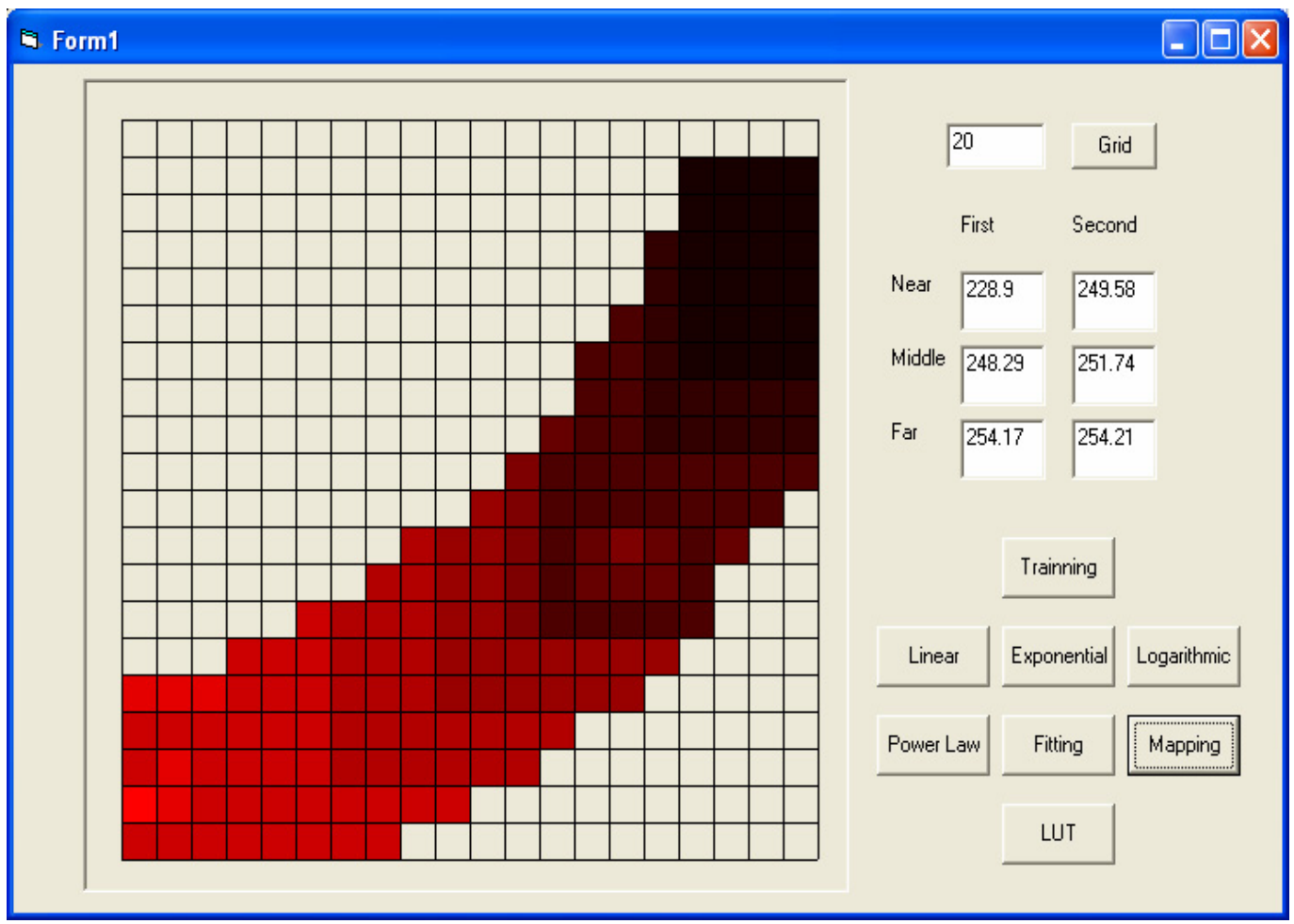

Fig.41 Fitting and Mapping of the Exponential Model

The developed program identified a point in the 2D space when a case was presented with two encoded input. The output was calculated according to the location of this point with respect to the trend curve and the assigned space around it. The fitness of the linear, exponential, logarithmic and power law estimation based on the simulation data is 
demonstrated in Fig.42. The performance of the estimation using linear model was presented in Table 4 by comparing the given and the estimated defect locations.

Table 4 Performance of the Linear Model

\begin{tabular}{|c|c|c|c|c|}
\hline $\begin{array}{c}\text { Second } \\
\text { Freq }\end{array}$ & $\begin{array}{c}\text { Third } \\
\text { Freq }\end{array}$ & $\begin{array}{c}\text { Given } \\
\text { Location }\end{array}$ & $\begin{array}{c}\text { Estimate } \\
\text { Location }\end{array}$ & $\begin{array}{c}\text { Err of } \\
\text { Range (\%) }\end{array}$ \\
\hline 228.9 & 249.58 & 20 & 20 & 0 \\
\hline 233.75 & 250.13 & 40 & 56 & 10 \\
\hline 239.6 & 250.63 & 60 & 73 & 8.125 \\
\hline 244.75 & 251.19 & 80 & 91 & 6.875 \\
\hline 248.29 & 251.74 & 100 & 109 & 5.625 \\
\hline 250.55 & 252.05 & 120 & 144 & 15 \\
\hline 252.24 & 252.71 & 140 & 162 & 13.75 \\
\hline 253.35 & 253.42 & 160 & 180 & 12.5 \\
\hline 254.17 & 254.21 & 180 & 180 & 0 \\
\hline
\end{tabular}

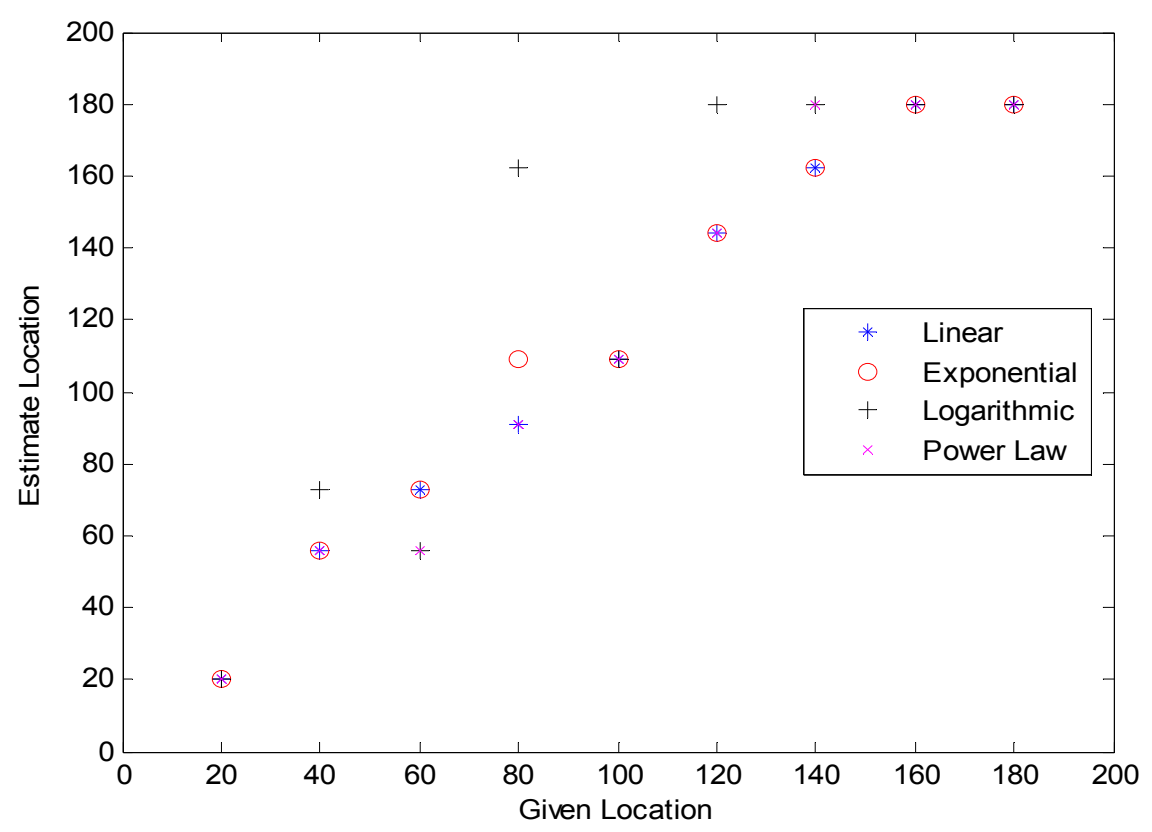


Fig.42 Comparison of the Accuracy of Four Different Models

The linear model had the most accurate estimations in this study; the error on average was $11 \%$. The error was calculated with respect to the range of the output and by considering only the test cases it never saw before.

\subsection{Conclusions}

In this chapter, the performance of the proposed IBR approach to implement SHM was investigated and simulations of the health monitoring for several systems were conducted. The models of simulated systems, such as a high displacement actuator, a turbojet engine, a four rotor helicopter, and their health monitoring systems using IBR were illustrated.

From the results of the simulated HDA and the engine, the IBR systems were easy to implement SHM concept, and in both cases, the IBR systems identified the simulated changes of the systems' status intermediately and correctly. Instead of using simple classifiers, encoders were added to estimate the step response of a highly non-linear system with an ARMAX time series model. It improved the accuracy of the evaluation of HIs during the transition period. For both simulations, it was possible to create very sophisticated health monitoring systems by using large number of IBRs to improve the performance. The preliminary modeling conducted in the studied cases yielded promising real life application guidelines. However, better design of the IBR classifiers would be needed.

For the simulation of the four rotor helicopter, IBR systems evaluated the conditions and presented HIs to the controllers. With the integration of health information, both PID and Fuzzy Logic control could adjust its strategy to maximize the performance of the 
helicopter. It was a tedious trial and error process to choose the parameters. Genetic algorithms may be applied to tune the parameters in future studies. The target in this study only considered vertical coordinate $\mathrm{z}$. In the future, $\mathrm{x}$ and $\mathrm{y}$ coordinate will be included.

From the results, it indicated that fuzzy logic was a fine choice to provide fault tolerant control. It was convenient to reconfigure the control surface with a set of rules designed according to expertise knowledge. Membership functions and rules need fine tune to optimize control parameters. As the classified types of health conditions increase, the number of rules will also increase dramatically, which will decrease the efficiency of the control scheme. Separate fuzzy logic controller is recommended if number of HI is greater than three. 


\section{CHAPTER VI}

\section{PERFORMANCE OF IBR ON EXPERIMENTAL DATA}

The previous simulations proved that the IBR is a powerful tool to implement SHM. In this chapter, IBR systems had been tested on experimental data. Experiments were carried out on carbon fiber tubes and helicopter rotors. Vibration based monitoring, Lamb wave based monitoring, and sweep sine based monitoring were applied to evaluate the structural integrity of the aforementioned structures. The purpose of these experiments was to explore the possibility of applying IBR on line to identify and locate damages in the system. Results indicated that IBR systems had potential to implement SHM for real time applications.

\subsection{Experiments on Composite Tubes}

According to the design of the DraganFlyer V ti Pro RC helicopter, the four rotors significantly improve the payload capability of the helicopter. On the other hand, the possibility of damages increases since it comprises several low-cost composite elements which support these rotors and the loads. In this study, the dynamic responses of carbonfiber tubes composing the main frame of the helicopter were investigated to estimate their structural integrity.

The carbon-fiber tubes are $190 \mathrm{~mm}$ long and the outer and inner diameters are $5 \mathrm{~mm}$ and $3 \mathrm{~mm}$ respectively. On some of the tubes, damages of different shapes and locations 
were made on purpose for control experiments. Fig.43 shows an example of a damaged carbon fiber tube with a through hole. Whereby, $\mathrm{X}$ indicates the distance from the fixed end to the hole and $\mathrm{D}$ indicates the diameter of the hole.

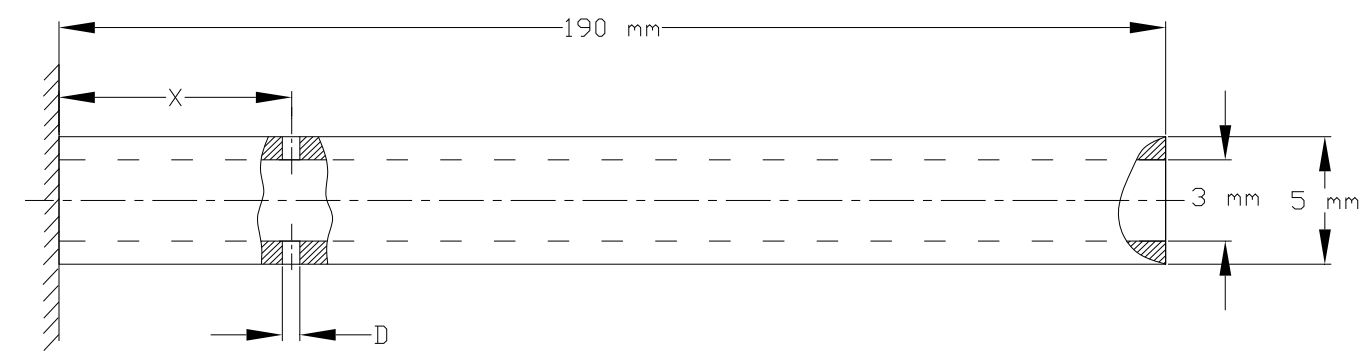

Fig.43 Dimensions of the Carbon Fiber Tube

\subsubsection{Vibration Based Monitoring}

In these experiments, the dynamic responses of carbon fiber tubes excited by the ambient vibration or generated signals were collected and analyzed. The relationship between the structural integrity of the tube and the dominant frequencies of the corresponding response spectrum was studied to explore the possibilities of classifying the damage of different sizes and different locations.

\subsubsection{Hole Damage}

First, two sets of carbon fiber tubes with a hole of $2.5 \mathrm{~mm}$ or $3 \mathrm{~mm}$ respectively were testified as cantilever beams. The location of the holes were $35 \mathrm{~mm}, 65 \mathrm{~mm}, 95 \mathrm{~mm}$, $125 \mathrm{~mm}$, or $155 \mathrm{~mm}$ away from the fixed end of the tube. The dimensions of the tubes are presented in Fig.44. 

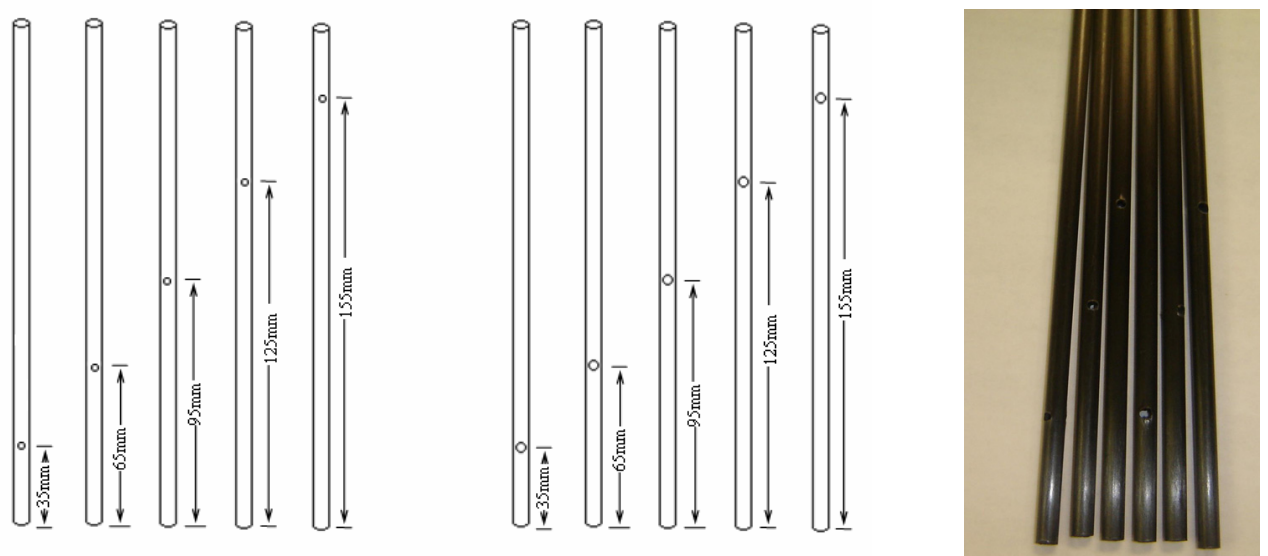

Fig.44 Dimensions of the Tubes with Defects (Left), and the Tubes (Right)

The vibration of the tubes was first measured by the laser vibrometer; the signal was then collected and evaluated by the Stanford Research Systems (SRS) Model SR780 2 Channel Signal Analyzer. The experimental setup was similar to that shown in Fig.46. Each tube of the two groups was fixed on the desk and its vibration was sensed by the reflection of the Doppler laser vibrometer beam.

The power spectrums of the tubes obtained by the spectrum analyzer are shown in Fig.45. The dominant frequency of the tube increased with the distance between the fixed end and the hole in all cases. The dominant frequency decreased with increasing hole size. The dominant frequencies and their amplitudes are listed in Table 5. 


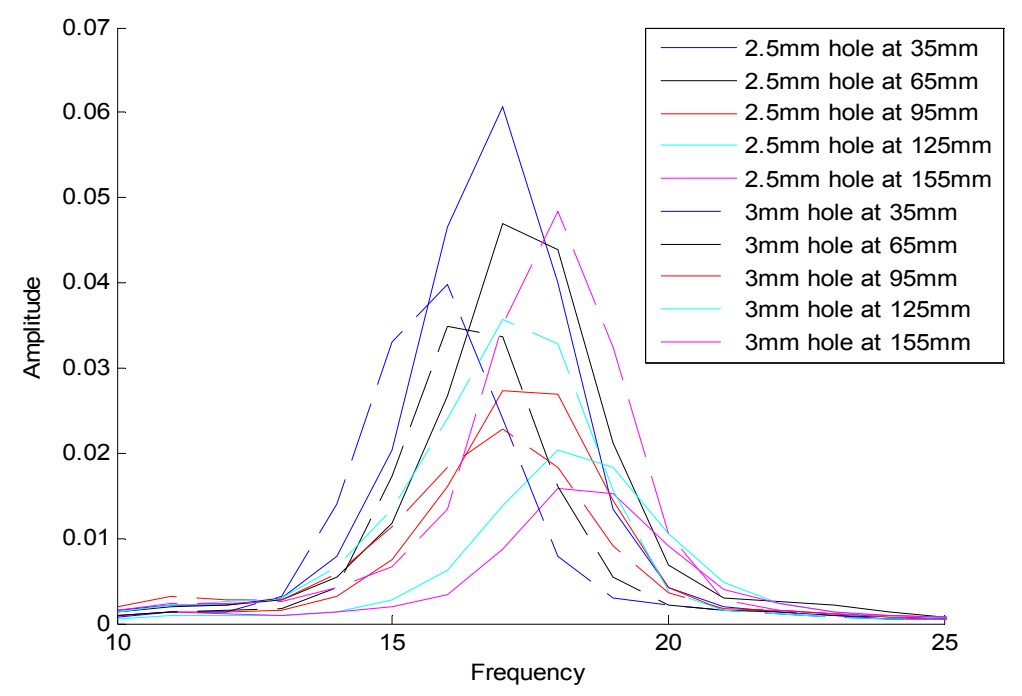

Fig.45 Frequency Responses of Tubes with a Hole of Different Size and Location

Table 5 Frequency Responses with Respect to Size and Location of Holes

\begin{tabular}{|c|c|c|c|}
\hline $\begin{array}{c}\text { Size } \\
(\mathrm{mm})\end{array}$ & $\begin{array}{c}\text { Distance } \\
(\mathrm{mm})\end{array}$ & $\begin{array}{c}\text { Frequency } \\
(\mathrm{Hz})\end{array}$ & $\begin{array}{c}\text { Amplitude } \\
(\mathrm{db})\end{array}$ \\
\hline \multirow{4}{*}{2.5} & 35 & 17 & 0.06059 \\
\cline { 2 - 4 } & 65 & 17.5 & 0.04699 \\
\cline { 2 - 4 } & 95 & 17.58 & 0.02735 \\
\cline { 2 - 4 } & 125 & 18.4 & 0.0204 \\
\cline { 2 - 4 } & 155 & 18.6 & 0.01593 \\
\hline \multirow{4}{*}{3} & 35 & 15.68 & 0.03968 \\
\cline { 2 - 4 } & 65 & 16.5 & 0.03485 \\
\cline { 2 - 4 } & 95 & 16.98 & 0.02267 \\
\cline { 2 - 4 } & 125 & 17.48 & 0.03569 \\
\cline { 2 - 4 } & 155 & 18 & 0.04831 \\
\hline
\end{tabular}

The whole arm of the Draganflyer helicopter was also testified, which included one carbon fiber tube and an electric motor with a propeller attaching to it. Other than the laser vibrometer, piezoelectric strips were also utilized to evaluate the vibration signals of the tubes. Piezoelectric strips are light, thin, portable and convenient to apply for real 
time applications. To evaluate their performance in identifying and locating the defect of structural elements, their dynamic frequency responses were compared with those of the laser vibrometer. The experimental setup is presented in Fig.46. One arm of the helicopter was fixed on the desk and the electric motor was connected to a power supply. A laser vibrometer was sit behind the helicopter branch, whose laser beam was perpendicular to the tube and it reflected at the reflecting tape attached near the top end of the tube. Another sensor, a piezoelectric strip actuator made by American Piezo Ceramics, Inc. (APCI) (Catalog No. 40-1035 (490/018/0.60-SA)) was attached on the surface of the tube near the free end. The dimensions of the piezoelectric strip were $49 \mathrm{~mm}$ in length, $2.1 \mathrm{~mm}$ in width and $0.6 \mathrm{~mm}$ in thickness. Signals from the laser vibrometer and the piezoelectric strip were collected and analyzed by the SRS signal analyzer.

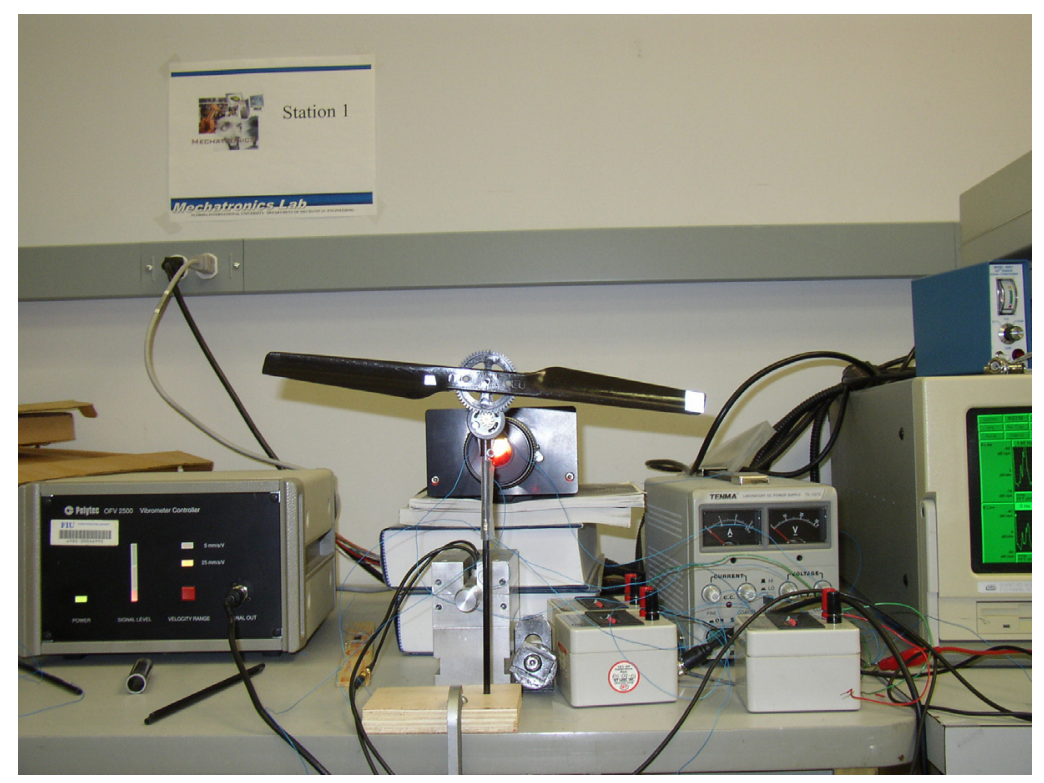

Fig.46 Experimental Setup to Monitor the Vibration of Composite Tubes

In the experiment, a perfect tube, a tube with a hole at the fix end (parallel to the laser beam), a tube with a hole at the free end (parallel to the laser beam) and a tube with a 
hole at the fixed end (perpendicular to the laser beam) were tested. The experiments were repeated while the motor was turned off or on. The diameter of the holes which were created to simulate the structural defects was $3 \mathrm{~mm}$.

The frequency responses of a perfect tube when the motor was not rotating, which were obtained from the signals collected by the laser vibrometer and the piezoelectric sensor, are presented in Fig.47. The dominant frequency of the perfect tube was indicated by the first spike of the spectrum which was located around $18 \mathrm{~Hz}$. In the plot, the spectrums of the signals obtained by both measurements were very similar. In other experiments, the similarity of the spectrums was also observed. The AC frequency of $60 \mathrm{~Hz}$ and its harmonics were also clearly noticeable in the plots. The same result can be found through sweep sine measurement shown in Fig.48.

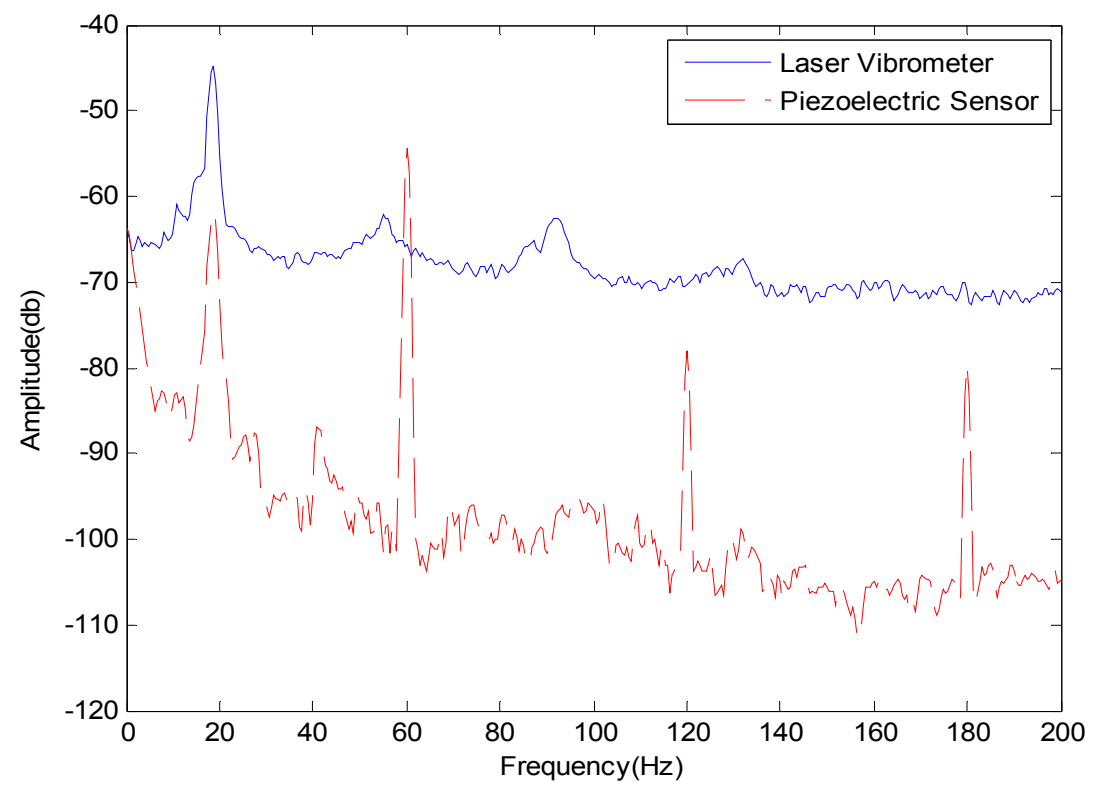

Fig.47 Frequency Responses of a Perfect Tube (Laser Vibrometer and Piezo) 


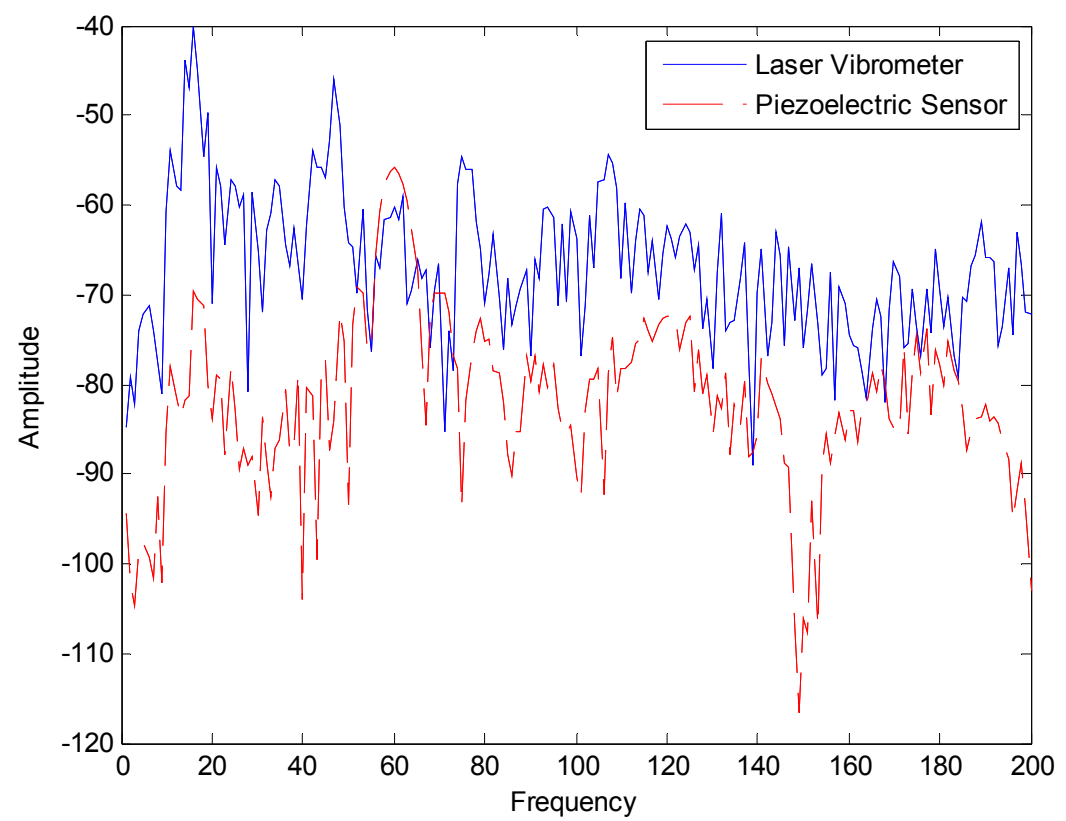

Fig.48 Sweep Sine Measurement of a Perfect Tube (Laser Vibrometer and Piezo)

The frequency responses of the defect tubes with a hole at different locations were obtained by the laser vibrometer, which are presented in Fig.49 and Fig.50. These plots indicated that the shift of the dominant frequency in the spectrum of the defective tube with a hole at the fix end from the dominant frequency of a perfect one could be easily noticed when the hole was parallel to the laser beam. The first frequency spike shifted to the left when the perfect tube was replaced with a defective one. In addition, the second and third harmonics varied more significantly than the first one. However, it was difficult to identify the difference from the spectral plots of the perfect tube and the defect one with a hole at the fix end perpendicular to the laser beam. The spectral shift was also little when the hole was at the free end of the tube, no matter if the hole is perpendicular or parallel to the laser beam. 


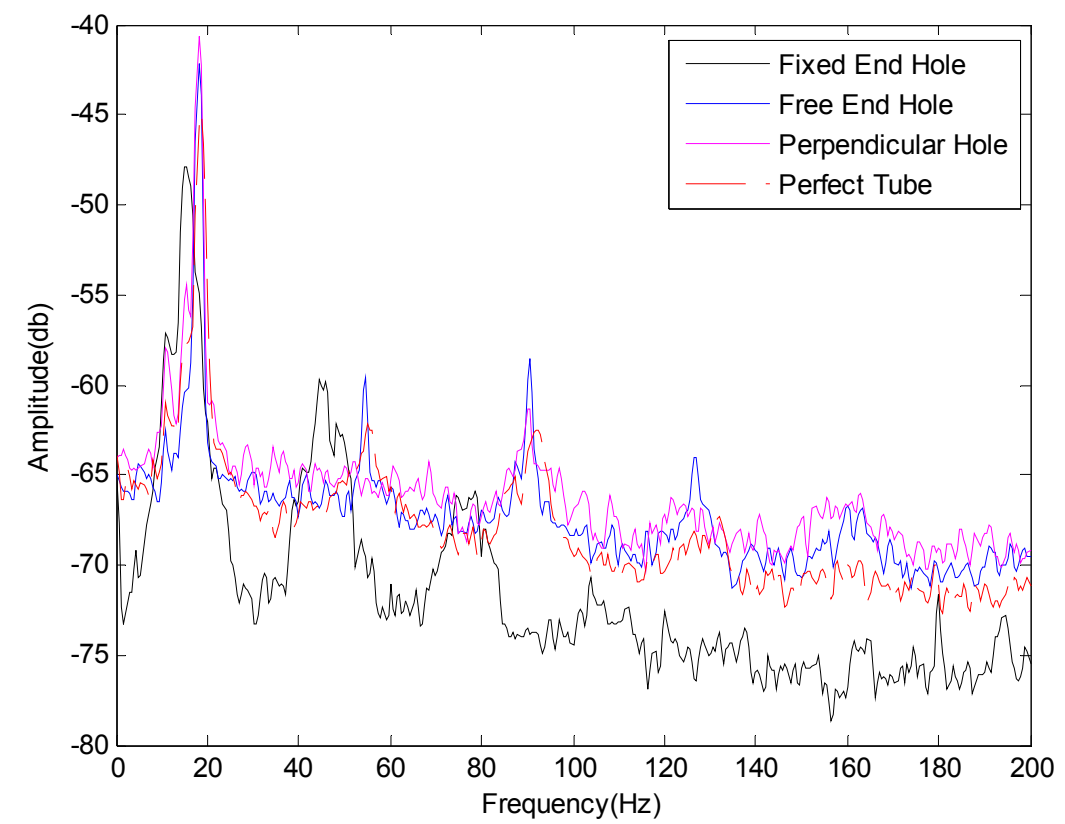

Fig.49 Frequency Responses of Perfect and Defect Tubes (Laser Vibrometer)

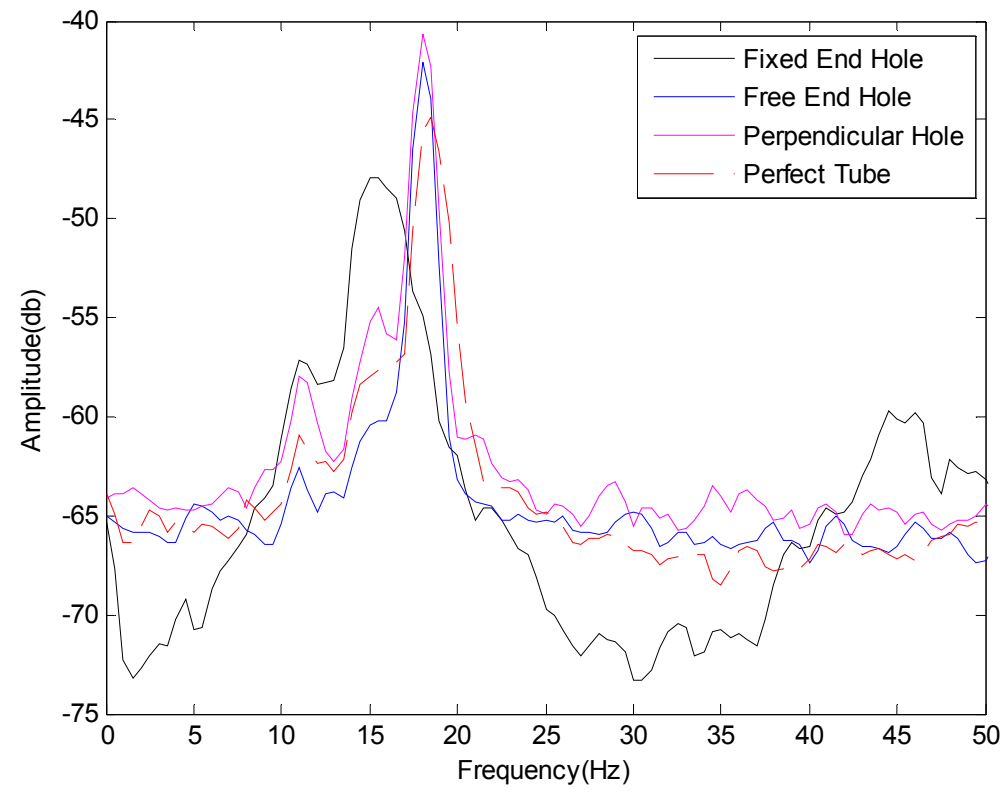

Fig.50 Low Frequency Part of Fig.49

When the electric motor started to operate, the spectrums become much more complex, since the spikes coming from the forced vibration of the motor and its 
harmonics were included. The spectrums are presented in Fig.51. It was difficult to distinguish the different modes of the frequency response from these combined signals. It was only possible to identify the perfect and damaged tubes from the spike located around $18 \mathrm{~Hz}$, since it appeared at the same frequency if the motor operates or not.

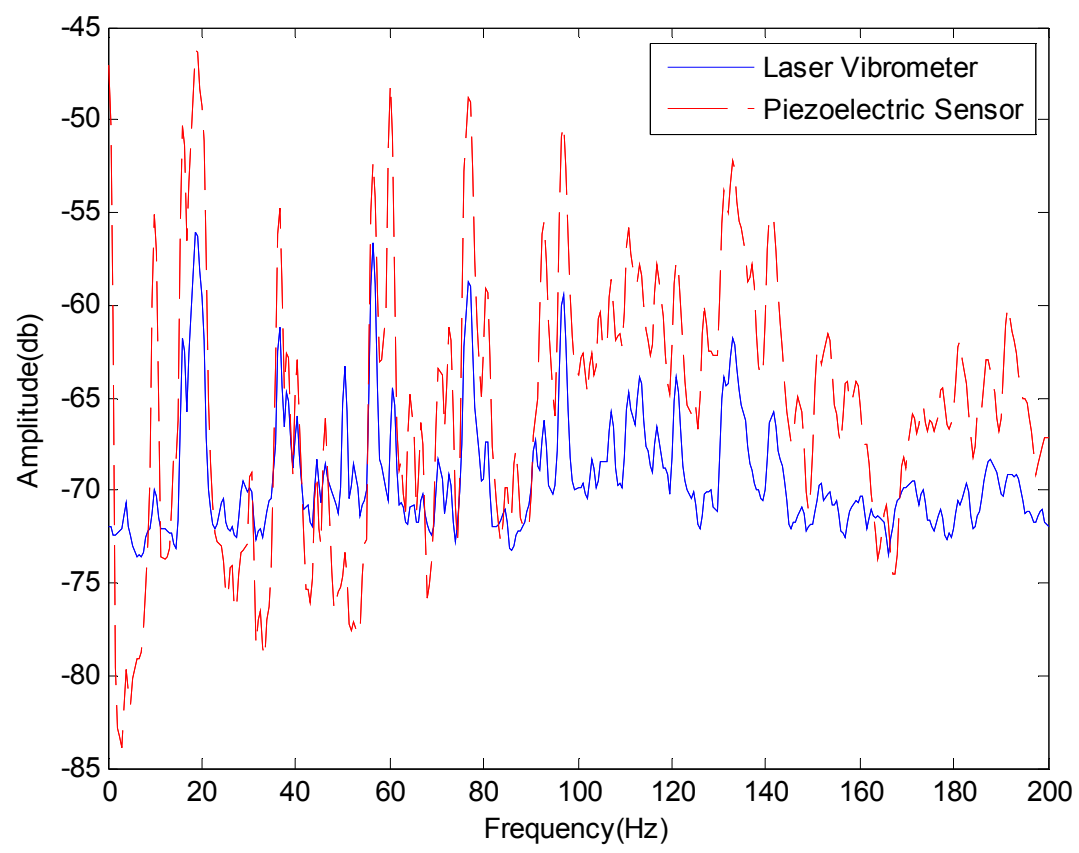

Fig.51 Frequency Responses of a Perfect Tube When the Rotor Was Running

From the experiments we can see that both laser vibrometer and the piezoelectric strip could be easily applied to obtain the frequency response characteristics of a structure. Laser vibrometer can acquire vibration signals from a remote location and it can be very accurate. However, most probably, it is utilized for laboratory experiments, because of its constraints of location and orientation in real time applications. Piezoelectric strip actuators were found a good fit for real time damage detection, since they are small, light and easy to be attached to the structure. Piezoelectric strips are also cheap, which make it possible to construct a sensor network embedded all over the system. In addition, the 
amplitude of the signals acquired by piezoelectric strips when the rotor was running was higher than that of the laser vibrometer without using any amplifying electronics in experiments.

The dynamic characteristics of the tubes, and the frequency responses when the rotors running at fixed RPM, were further analyzed. The piezoelectric strips were attached on the tube of a detached arm and an arm of the whole helicopter to identify the existence of damages. The experiment set up is shown in Fig.52 and Fig.53 respectively.

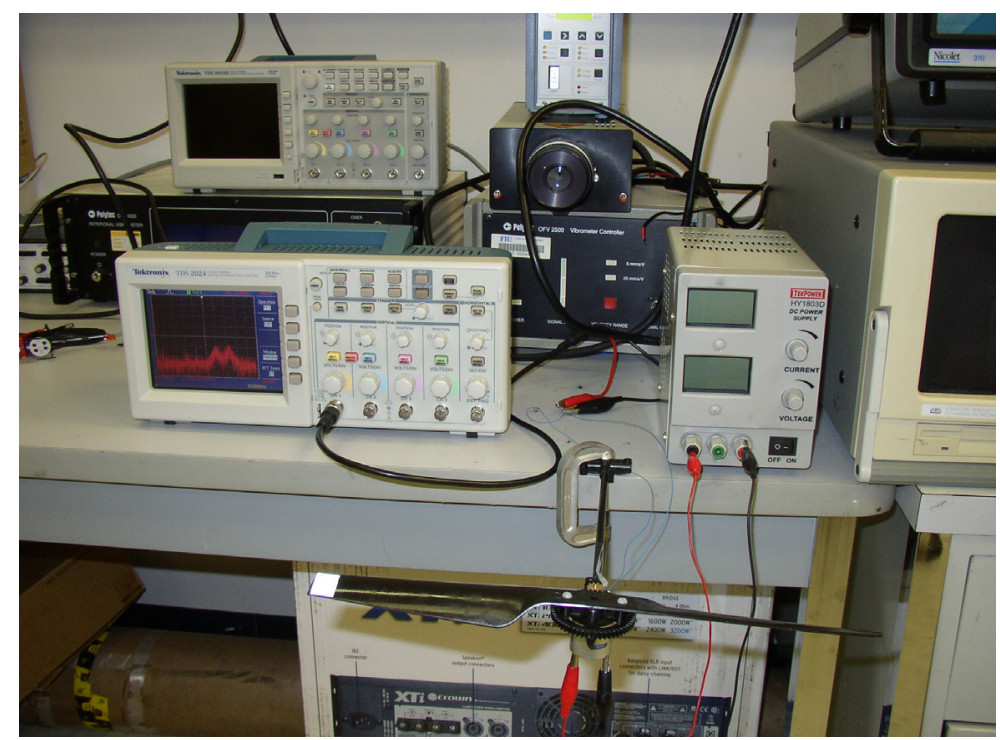

Fig.52 Experimental Setup (Rotor of One Arm Was Running at Fixed RPM) 


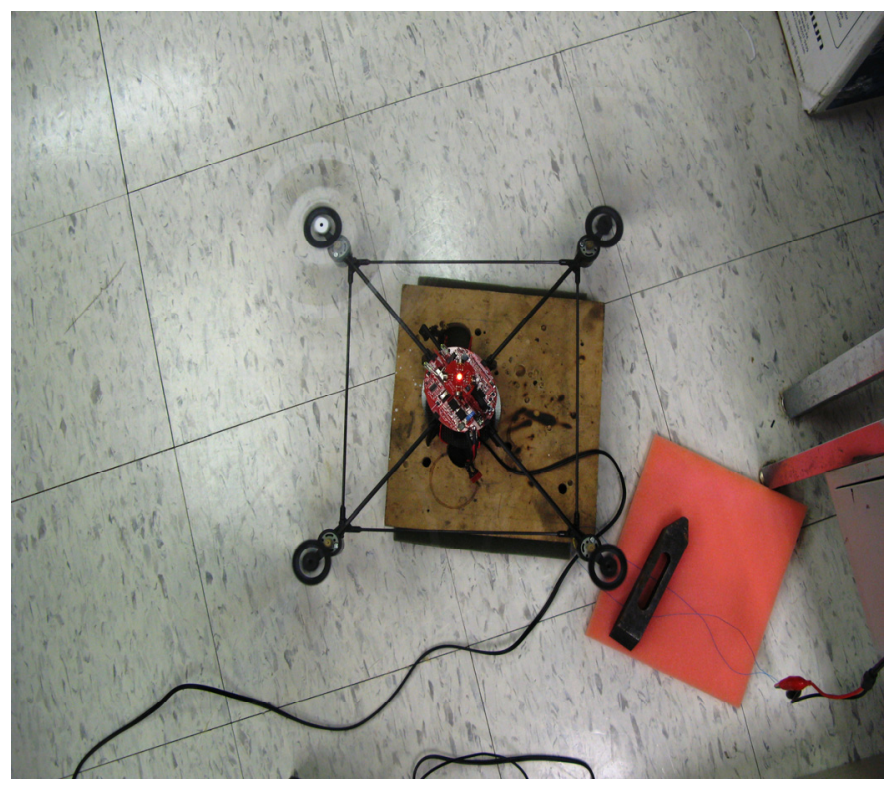

Fig.53 Experimental Setup to Identify Damages of One Arm (Helicopter Was Running)

It was easy to control the rotor of the detached arm element rotating at fixed RPM by supplying certain amount voltage, but it was quite difficult to make the whole helicopter run at fixed RPM. Since the movements of all the rotors are coupled with each other due to the design of the four rotor helicopter. The voltage allocated to each rotor may change automatically according to the reading of sensors, the voltage of the battery, etc.

For both cases, it was quite difficult to distinguish the frequency shift due to damages of the structures. The dynamic responses of the perfect and the damaged tubes, when the whole helicopter was running at low RPM and high RPM, are shown in Fig.54 and Fig.55. 


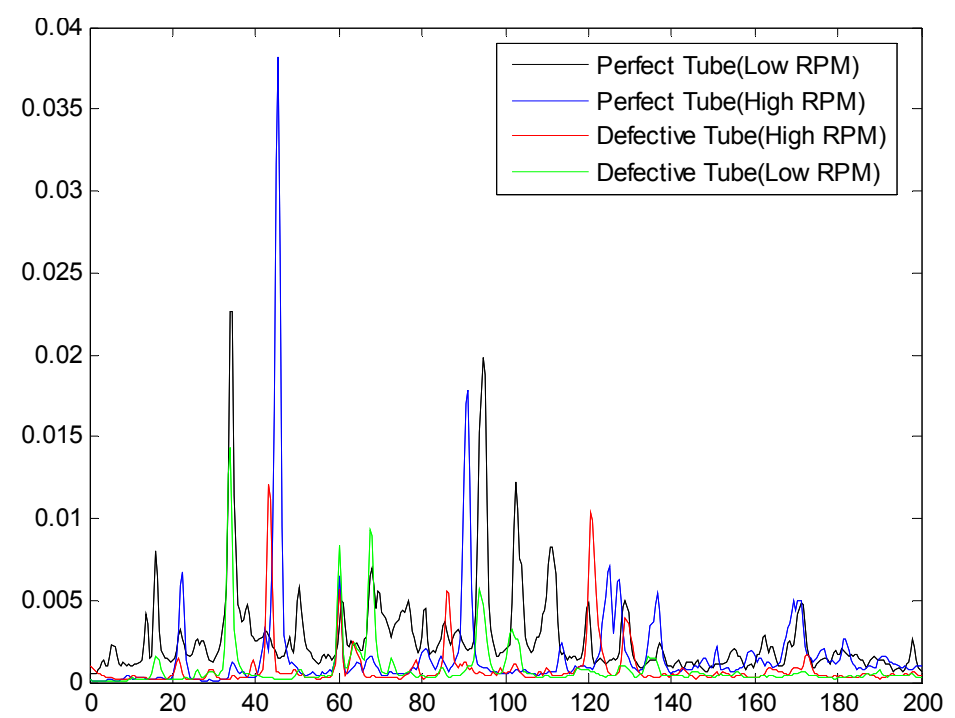

Fig.54 Dynamic Responses of Perfect and Defect Tubes When the Rotor Was Running at Low RPM and High RPM

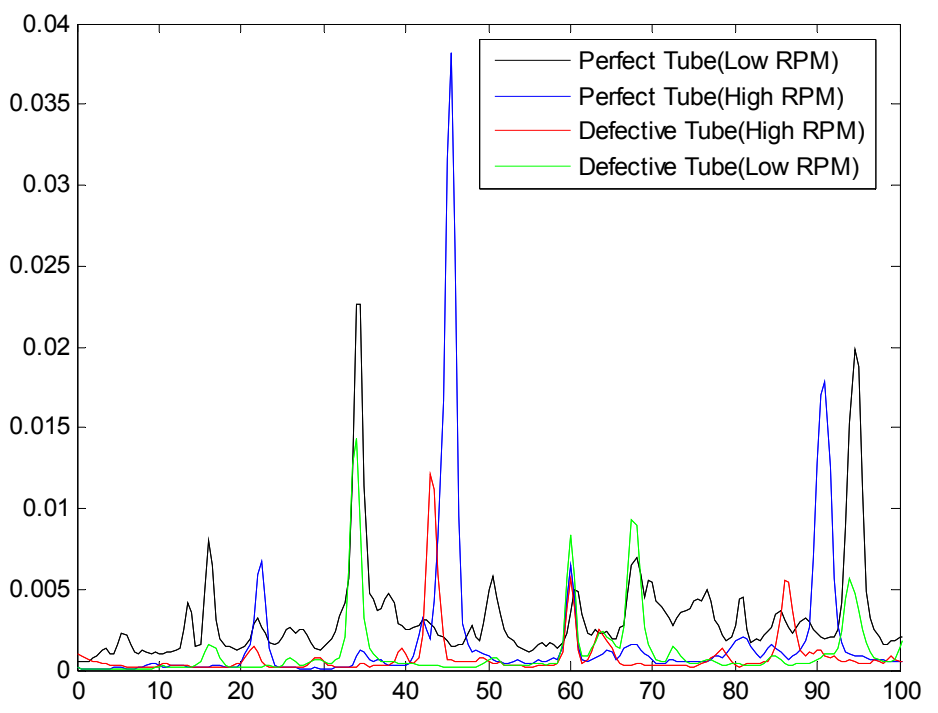

Fig.55 Low Frequency Part of Fig.54

\subsubsection{Slot Damage}

Vibration test system was also utilized to analyze the characteristics of the tubes through exciting them with the sweep sine wave. The diagram of the experimental setup is presented in Fig.56. One arm of the helicopter was attached to a V6100-6 VT5 
vibration test system. The excitation signal was generated by the SRS signal analyzer. It then was connected to the MacroTech Crown XTI 1000 power amplifier to amplify the signal presented to the dynamic exciter. The sweep sine wave and the white noise were applied as the excitation signals in the experiments. The vibration responses measured by the piezoelectric strip bonded on the tube was acquired by the same spectrum analyzer which generated the excitation signal.

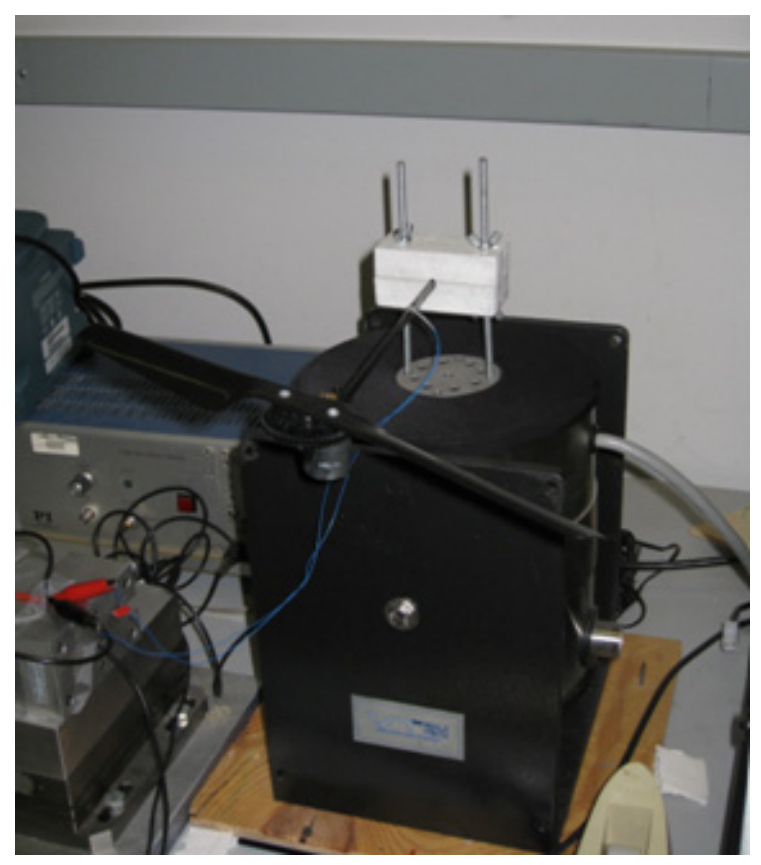

Fig.56 Experimental Setup (Vibration Test System)

The perfect and the damaged tubes were used in the experiments. The damages were created at two different locations as presented in Fig.57. One of the damage was very close to the fix end and the other one was in the middle of the tube. The depth of the slot damage was $1 / 2$ of the diameter of the tube. 


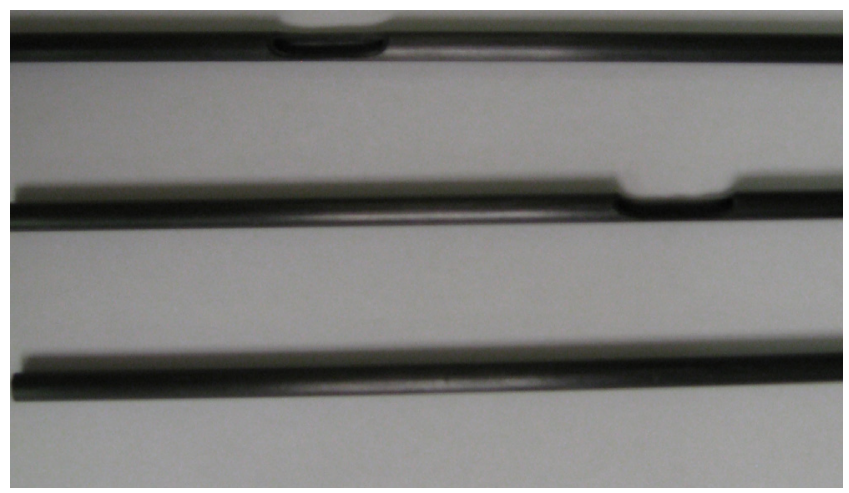

Fig.57 Perfect Tube and Damaged Tubes with the Slot

To enrich the classification performance of IBR, self organizing abilities was integrated into IBR nodes. A self organizing map was built and trained with the frequency responses of damaged and undamaged tubes to learn the different patterns. The Simulink model of this approach is demonstrated in Fig.58. Two sets of the perfect and the damaged tubes were tested on the vibration system to collect the data. Since it was impossible to get more damaged tubes, simulation data were also generated to evaluate the performance of this approach. The frequency responses of different tubes are presented in Fig.59. The training data set to be presented to SOM was extracted from the second and the fourth groups of peaks.

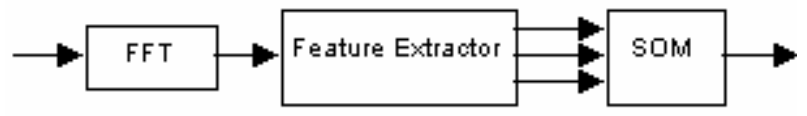

Fig.58 Simulink Model of the Proposed IBR+SOM Approach 


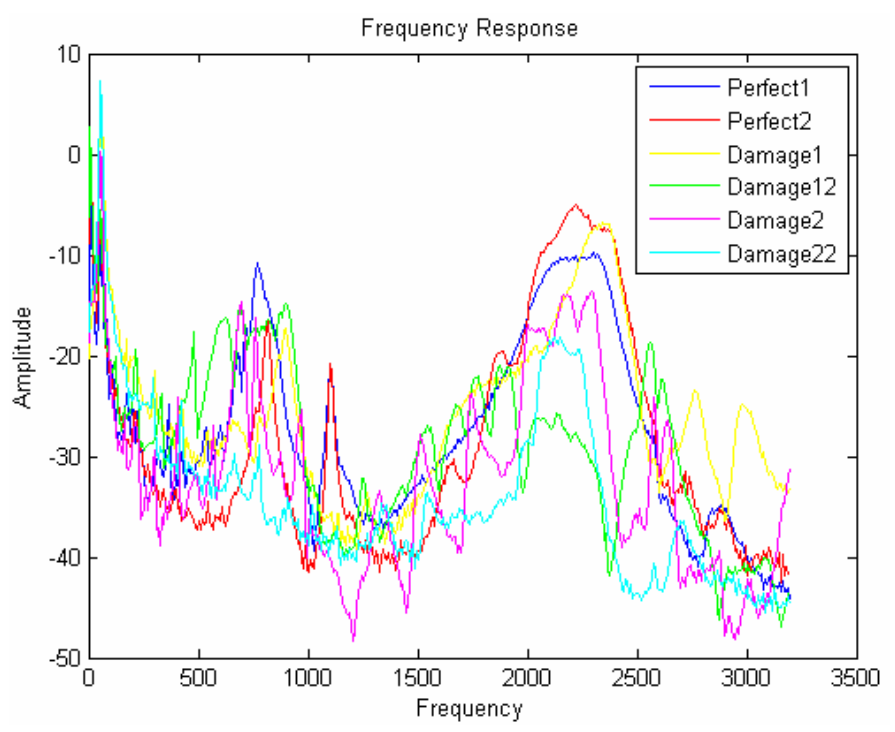

Fig.59 FFT Results of Tested Tubes

To generate the SOM models, the Neural Networks Toolbox of MATLAB ${ }^{\circledR}$ was used. The classification accuracy of the SOM depends on the selected lattice; in this study, the output map was laid out in a $10 * 10$ grid topology. The Euclidean distance measurement was chosen. The learning rate was 0.5 and the iterations were allowed to reach to 2000 epoch. The initial weights were randomly assigned. The training result is presented in Fig.60.

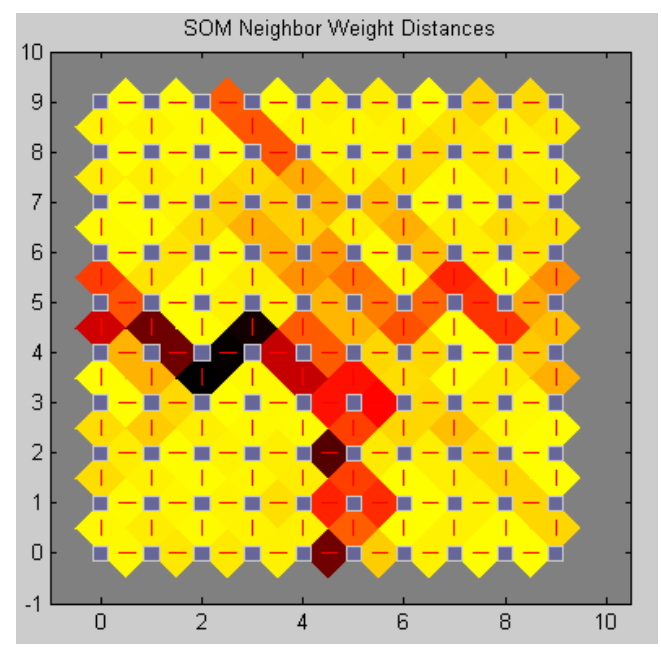

Fig.60 SOM Training Results 
The colors in this picture indicated the distance or dissimilarity of each neuron. The light colors meant the distance was short and the neurons were clustered together. While the dark colors indicated that the neurons were far away from each other, which should be the boundary of different clusters. The sample hit graph in Fig.61 shows how the input data was associated with each neuron of the output map.

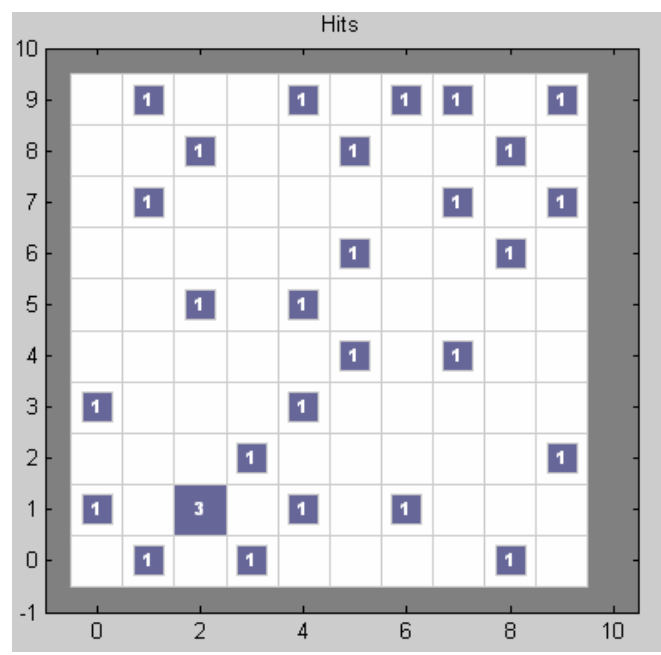

Fig.61 Sample Hits

The learning process of SOM assigned training vectors carefully to create clusters. Each neuron was associated with one cluster of the output map. Since the training data included the information of class types, in this case, the different location of the defect on the carbon fiber tube, the clusters could be labeled with the class types. So, the neurons representing the sample data could be classified. Fig.62 illustrates the clusters with the labels referring to the defect types. Light blue neurons represented type 1, which was the perfect tube. Yellow neurons represented type 2, with the damage hole at the fixed end of the tube, while the red neurons represented type 3, with the damage in the middle of the tube. 


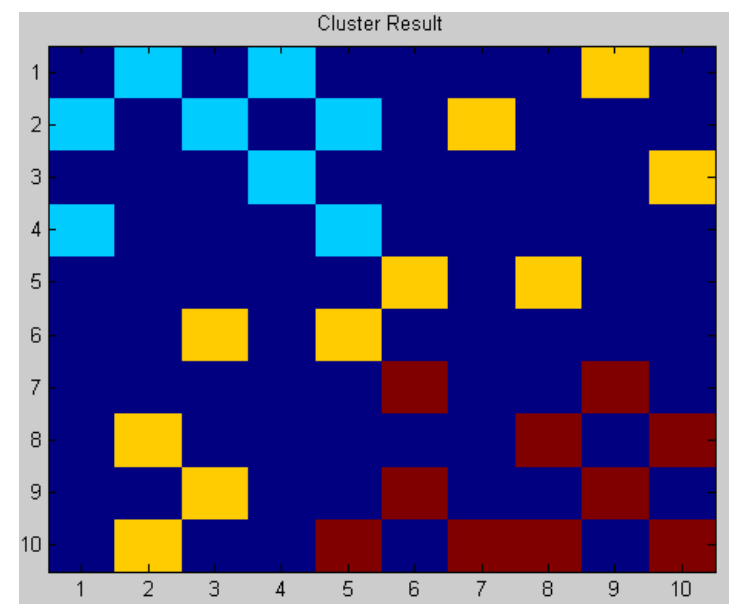

Fig.62 Clusters Labeled with Different Types

To implement SOM into the IBR as a classifying LUT, those unlabeled neurons had to be also assigned with a class number. Here, the Euclidean distance of the unlabeled neurons and the surrounding labeled neurons was calculated, the class type of the nearest labeled neuron was then assigned to the unlabeled neurons. The LUT in Fig.63 was prepared for classification of the test data. The result of the test is presented in Table 6 .

\begin{tabular}{|c|c|c|c|c|c|c|c|c|c|c|c|}
\hline \multicolumn{9}{|c|}{ table } & \multicolumn{3}{|c|}{$-\square \pi x$} \\
\hline \multicolumn{12}{|c|}{$\boxplus$ table $<10 \times 10$ double $>$} \\
\hline & 1 & 2 & 3 & 4 & 5 & 6 & 7 & 8 & 9 & 10 & \\
\hline 1 & 1 & 1 & 1 & 1 & 1 & 2 & 2 & 2 & 2 & 2 & $\hat{\wedge}$ \\
\hline 2 & 1 & 1 & 1 & 1 & 1 & 2 & 2 & 2 & 2 & 2 & \\
\hline 3 & 1 & 1 & 1 & 1 & 1 & 1 & 2 & 2 & 2 & 2 & \\
\hline 4 & 1 & 1 & 1 & 1 & 1 & 2 & 2 & 2 & 2 & 2 & \\
\hline 5 & 1 & 2 & 2 & 2 & 2 & 2 & 2 & 2 & 2 & 3 & \\
\hline 6 & 1 & 2 & 2 & 2 & 2 & 2 & 2 & 2 & 3 & 3 & \\
\hline 7 & 2 & 2 & 2 & 2 & 3 & 3 & 3 & 3 & 3 & 3 & \\
\hline 8 & 2 & 2 & 2 & 2 & 3 & 3 & 3 & 3 & 3 & 3 & \\
\hline 9 & 2 & 2 & 2 & 2 & 3 & 3 & 3 & 3 & 3 & 3 & \\
\hline 10 & 2 & 2 & 2 & 3 & 3 & 3 & 3 & 3 & 3 & 3 & \\
\hline 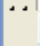 & & & & & & & & & & & $>$ \\
\hline
\end{tabular}

Fig.63 Look Up Table 
Table 6 Test Results of SOM

\begin{tabular}{|c|c|c|c|c|c|c|c|c|c|c|}
\hline Sample Index & 1 & 2 & 3 & 4 & 5 & 6 & 7 & 8 & 9 & 10 \\
\hline Assigned Type & 1 & 1 & 1 & 1 & 1 & 1 & 1 & 1 & 1 & 1 \\
\hline Classified Type & 2 & 1 & 1 & 1 & 1 & 1 & 1 & 1 & 1 & 2 \\
\hline Sample Index & 11 & 12 & 13 & 14 & 15 & 16 & 17 & 18 & 19 & 20 \\
\hline Assigned Type & 2 & 2 & 2 & 2 & 2 & 2 & 2 & 2 & 2 & 2 \\
\hline Classified Type & 2 & 2 & 2 & 2 & 2 & 3 & 3 & 2 & 1 & 3 \\
\hline Sample Index & 21 & 22 & 23 & 24 & 25 & 26 & 27 & 28 & 29 & 30 \\
\hline Assigned Type & 3 & 3 & 3 & 3 & 3 & 3 & 3 & 3 & 3 & 3 \\
\hline Classified Type & 3 & 3 & 3 & 3 & 3 & 2 & 3 & 3 & 3 & 3 \\
\hline
\end{tabular}

\subsubsection{Sweep Sine Based Monitoring}

To apply the previous approach in real time monitoring, the vibration test system was replaced with a piece of piezoelectric actuator to excite the carbon fiber tube. The experiment setup to evaluate the health condition of the composite tube is presented in Fig.64. Two piezoelectric elements manufactured by the American Piezo Ceramics, Inc. (APCI) (Catalog No. 40-1035 (490/018/0.60-SA)) were attached to the two opposite ends of the tube, which is shown in Fig.65. The diameter of the $1.1 \mathrm{~mm}$ thick piezoelectric elements is $6.4 \mathrm{~mm}$. One of the piezoelectric disks was utilized as an actuator and the other one was utilized as a sensor. The perfect and the damaged carbon fiber tubes were excited by the actuators and their responses were measured at the other end with the identical sensors. The actuators were excited with a sweep sine wave from $0 \mathrm{~Hz}$ to $100 \mathrm{KHz}$. The sweep sine wave was generated by the SRS signal analyzer which also obtained the frequency domain characteristics of the voltage generated by the other piezoelectric element according to the excitation. First, a perfect tube was excited and the frequency response was collected. Then the same experiment procedure was repeated on 
the damaged tubes with a slot as deep as $1 / 4$ or $1 / 2$ of the tube's diameter to acquire the vibration signals.

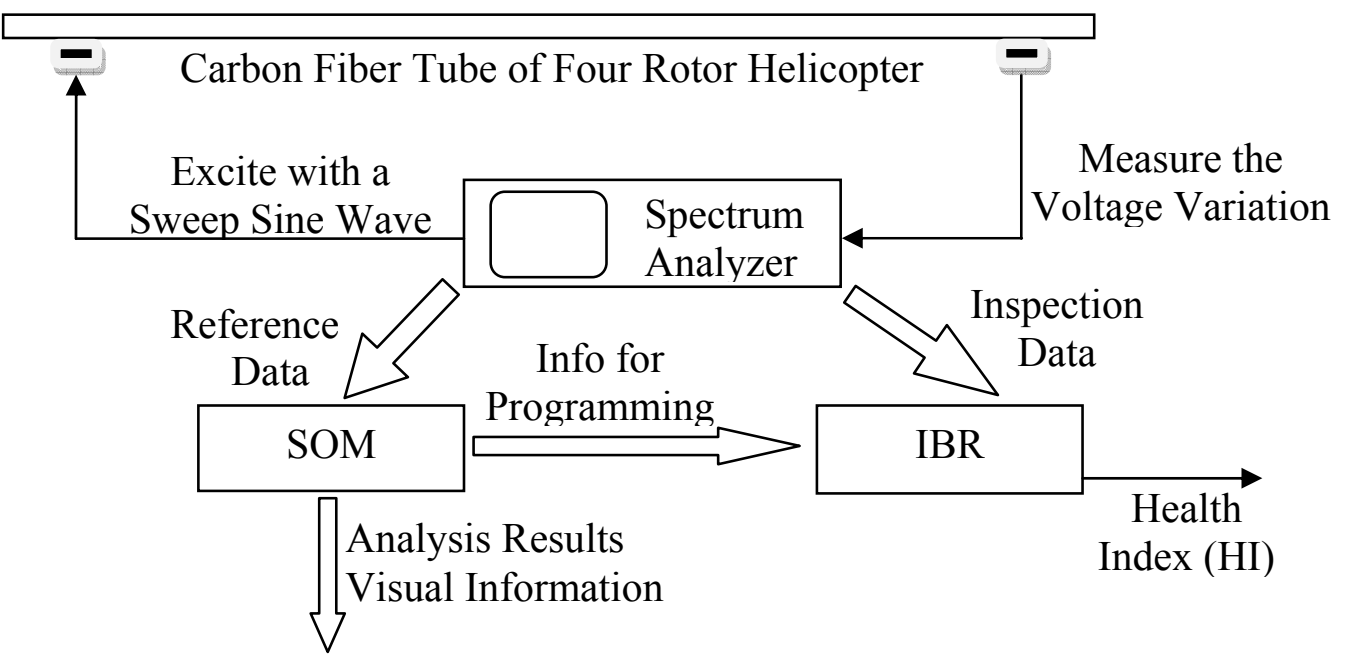

Fig.64 Proposed Procedure Using SOM-IBR Combination for Classification

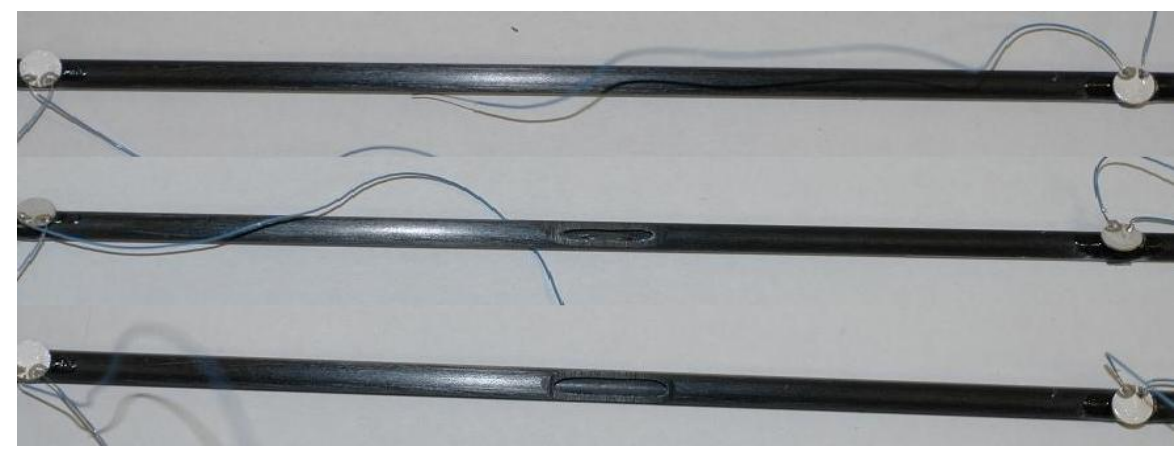

Fig.65 Perfect and Damaged Tubes Bonded with Piezoelectric Actuators and Sensors

The dynamic responses were then analyzed by SOM to determine the quality of the data, to generate classes, to visualize the characteristics of the data and to obtain the guidelines for preparing the LUT of the IBR nodes. The programmed IBR evaluated the encoded parameters of the incoming data and calculated the HIs for estimation of the structural integrity. 
The experimentally obtained frequency responses in the $0 \mathrm{~Hz}$ to $100 \mathrm{kHz}$ range are presented in Fig.66. The drastic drops in the frequency response plot of the perfect tube were the most distinctive differences from the frequency response plots of the damaged tubes. The frequencies and magnitudes of the five drastic drops in the spectrum of the perfect tube and the according information of the damaged tubes were identified and used for classification purpose. Since the dynamic responses of tubes were very repetitive, synthetic data was generated around the encoded parameters of the three original cases corresponding to one perfect and two damaged tubes.

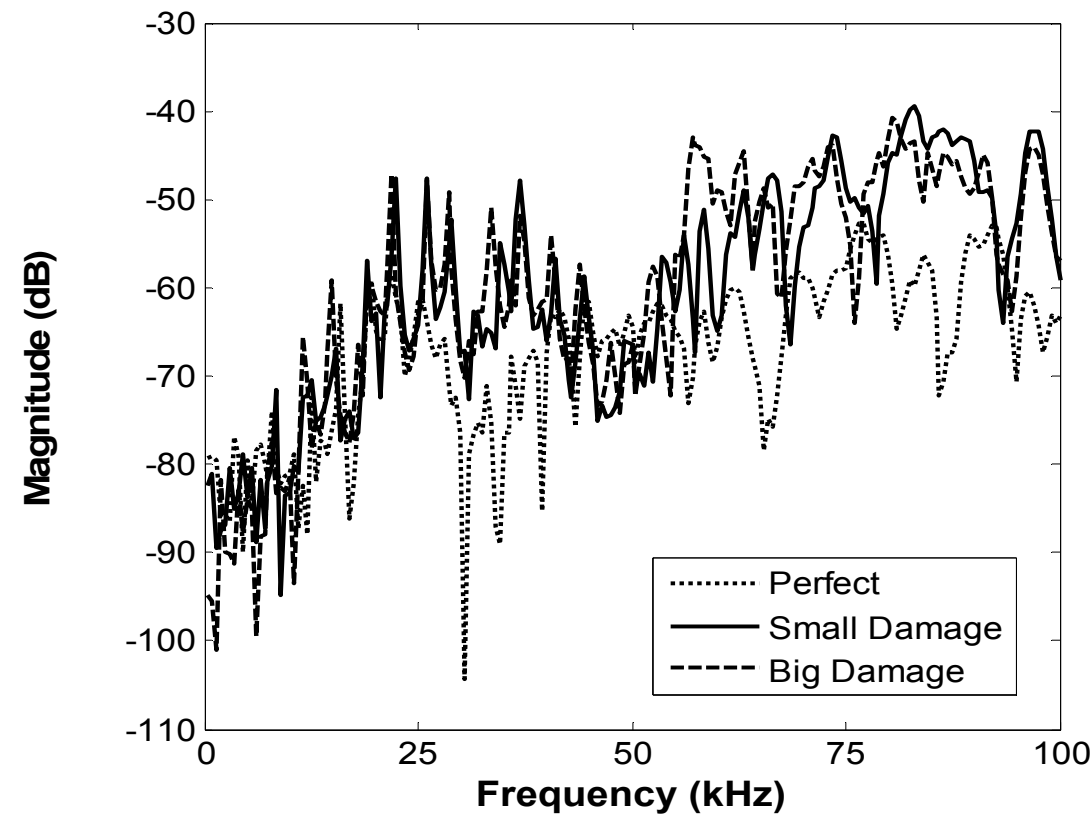

Fig.66 Sweep Sine Responses of Perfect and Damaged Tubes

99 cases of encoded frequency domain characteristics of damaged and undamaged tubes were submitted to the SOM network generated by MATLAB® Neural Networks Toolbox. 84 additional cases were used for test. 
In this study, 10 x10 grid layout was used. The link distance function was chosen to calculate the distance of neurons. The ordering phase learning rate and the tuning phase learning rate were selected as 0.9 and 0.5 respectively. The step was 1000 and the neighborhood distance was 0.5 . The iterations were 1000 epoch.

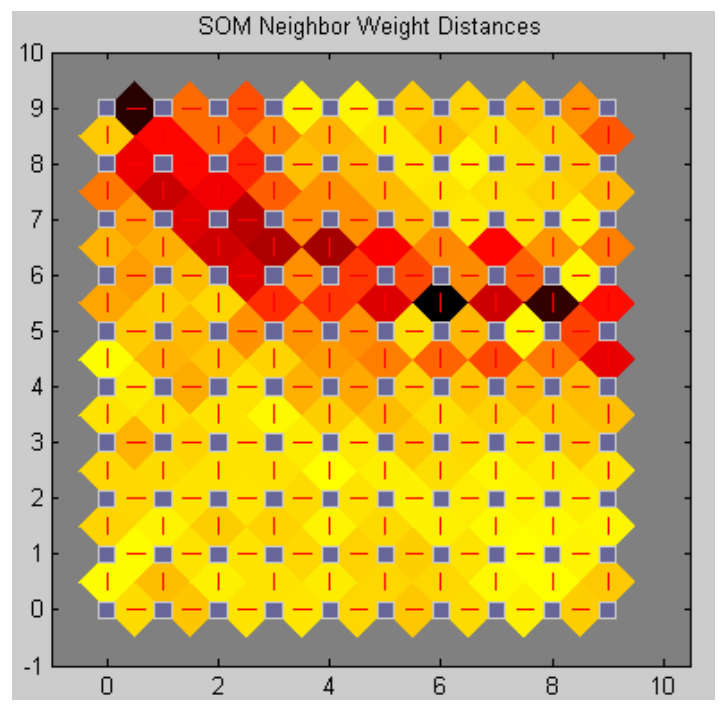

Fig.67 Visualization of the Neighbor Weight Distances after the Training

The display of the SOM neighbor weight distances after the analysis of the training data is presented in Fig.67. As in the previous case, the neurons representing the similar characteristics were clustered together. From the graph, a dark color belt which indicated the boundary of different clusters divided the map into two subspaces. The neuron positions and their weights are illustrated in Fig.68. It is clear that one group of neurons were far away from the others while two other groups located quite close to each other. This observation was very similar to the position of the encoded parameters of the perfect and the defect tubes in the frequency spectrums. These indicated that the frequency characteristics of the perfect tube was different from those of the other tubes with two different levels of damages. 


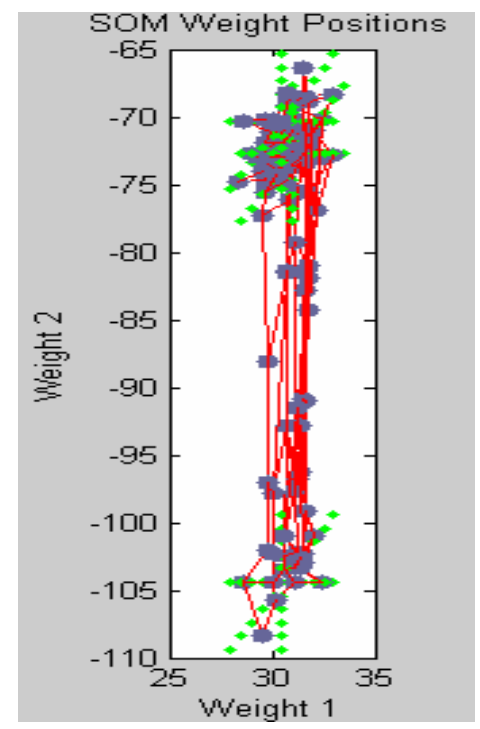

Fig.68 Weight Positions of the SOM

After associating the neurons representing the training data with the health conditions of the tubes, HI values could be assigned to different clusters. Fig.69 presents the assigned HI values to the different clusters. The light blue neurons represented the cases with $\mathrm{HI}=1$. The related data was collected by using a perfect tube. The yellow neurons represented the cases with $\mathrm{HI}=2$. The frequency response information was obtained by using a tube with the small damage. The brown neurons represented the $\mathrm{HI}=3$ and corresponded to the big damage. The dark blue neurons were not associated with any input data, so their class types were unknown. The unknown neurons were assigned the HI of the nearest labeled neuron. The prepared IBR LUT utilized to estimate the HI value for the incoming testing data is demonstrated in Fig. 70 


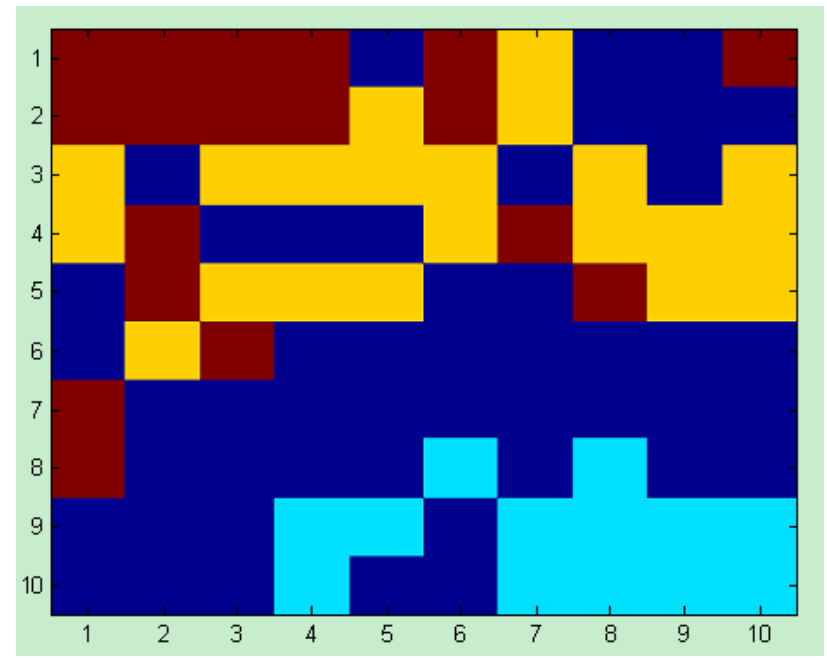

Fig.69 Four Clusters Corresponding to Different Structural Integrity Levels

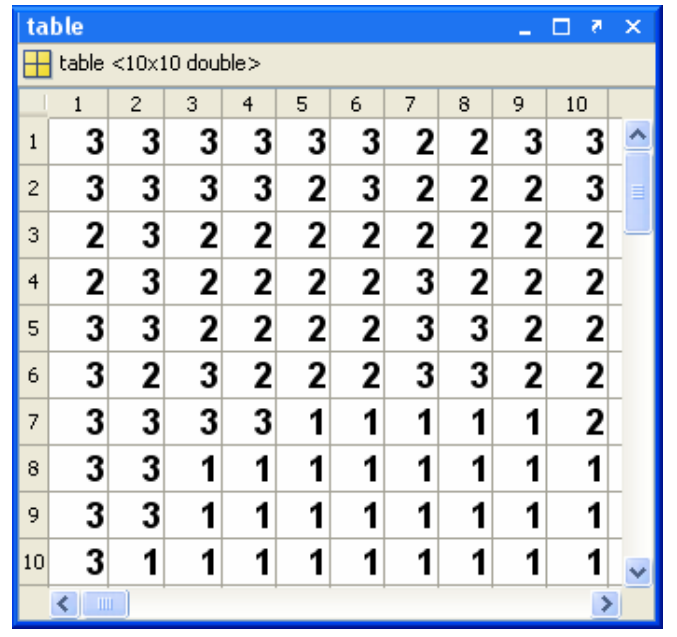

Fig.70 Look Up Table

The test results are presented in Fig.71. The encoded parameters of the perfect and the damaged tubes were always distinguished with $100 \%$ accuracy. Since the characteristics of the data with the small and the large damages were very similar, IBR classified the severity of the damage with $84 \%$ accuracy in the 84 test cases. 


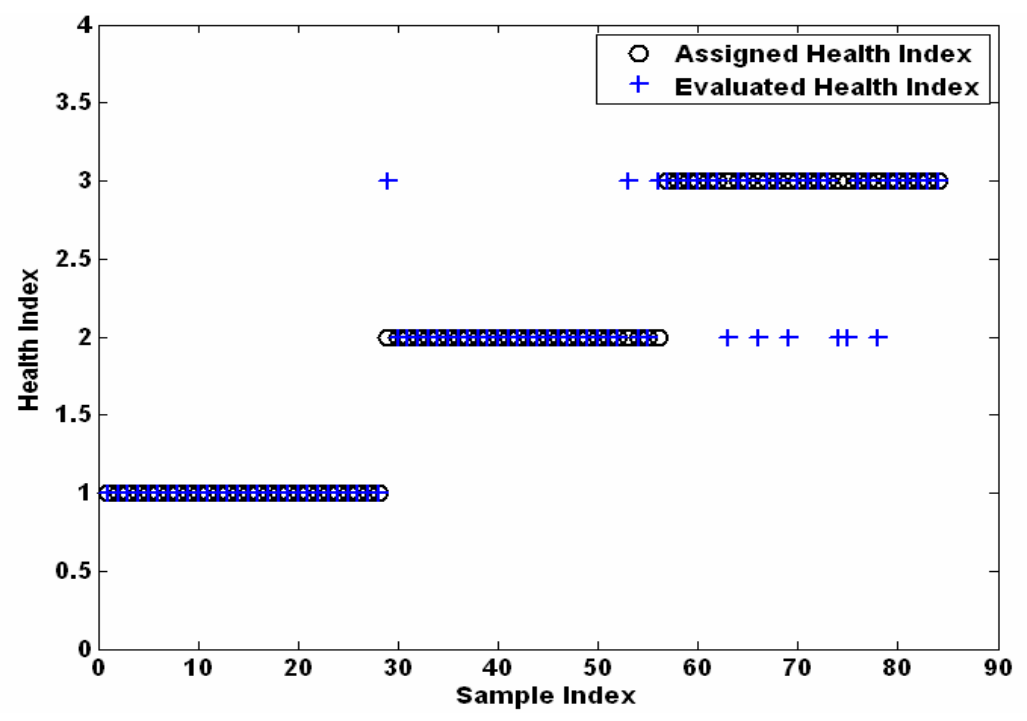

Fig.71 Test Results

In this experiment, the stress change of the carbon fiber tube was also evaluated through the surface waves propagated along the tube. In stead of damaging the tube, a clamp was attached to the tube. This allowed us to repeat the experiments attaching the clamp at different locations and with different tightness using only one tube. As the previous experiment, the piezoelectric attached at one end of the tube generated a chirp signal from $0 \mathrm{~Hz}$ to $102.4 \mathrm{KHz}$ and the piezoelectric sensor at the other end measured the dynamic responses. Since the property change of the tube and the influence of an obstacle would disturb the waves propagating along the surface of the tube, through analyzing the propagation changes, the structural and the assembly problems of the tube could be detected. The experimental setup is presented in Fig.72. The same experimental procedure was repeated when the clamp was attached at $40 \mathrm{~mm}, 80 \mathrm{~mm}$ or $120 \mathrm{~mm}$ away from the actuator. 


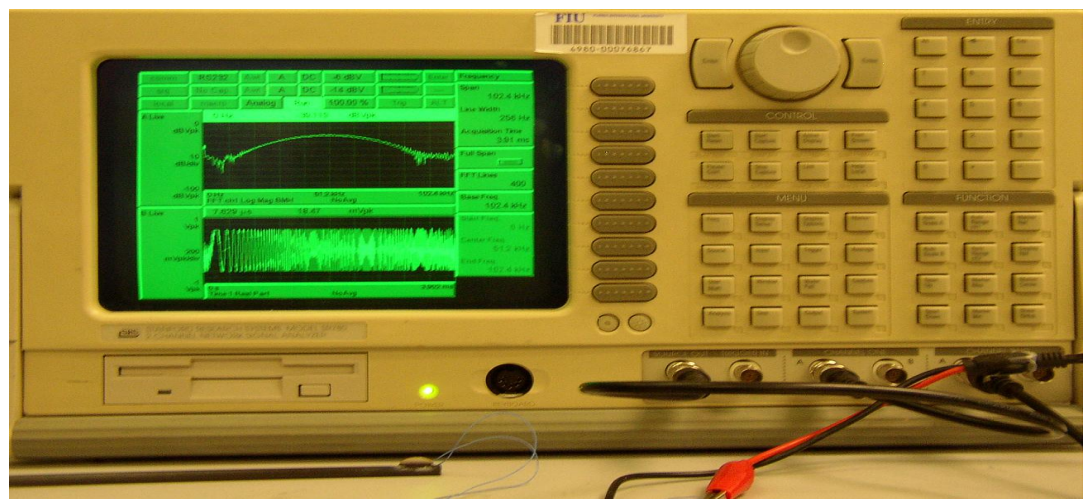

Fig.72 Experimental Setup for Sweep Sine Based Monitoring

The $\mathrm{S}$ transform of the propagated waves received at the sensor side was utilized to extract and encode the features indicating the condition of the structure. The characteristics of the $\mathrm{S}$ transforms of the propagated waves varied according to the property changes which influenced the propagation of surface waves. Fig.73 shows the S transforms of the free tube and the same tube when the clamp was placed at three different locations. In Fig.73, the time-frequency characteristics of the sensory signals of the tube under the aforementioned conditions were illustrated by contours of the $\mathrm{S}$ transforms. The contours represented certain amplitude levels. Fig.73 indicated that the S transform contours of the free tube and the same tube with a clamp had different characteristics. The free tube had the higher spikes since the clamps obstructed the propagation of the surface waves and reduced their energies. 


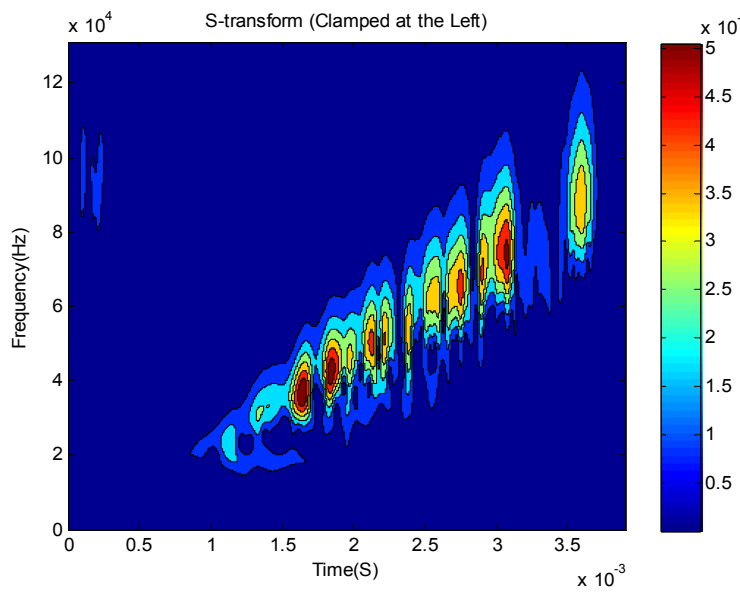

a) Free Tube

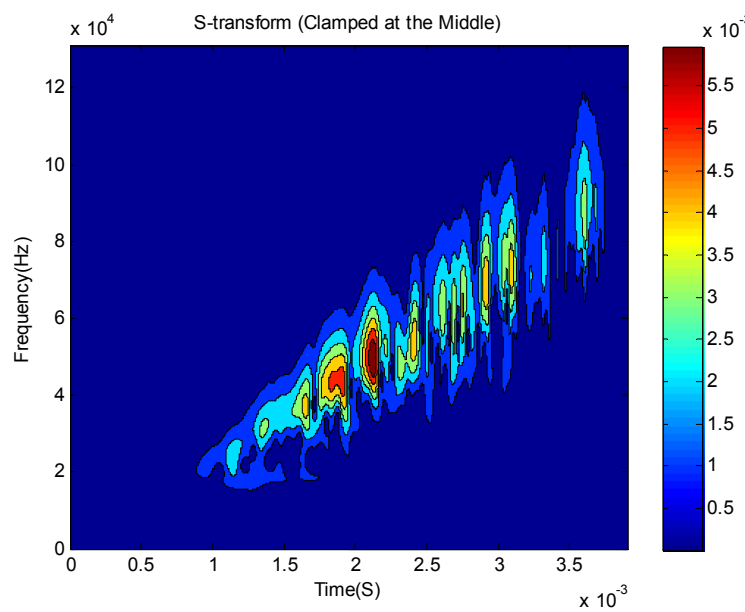

c) Clamped at the Middle

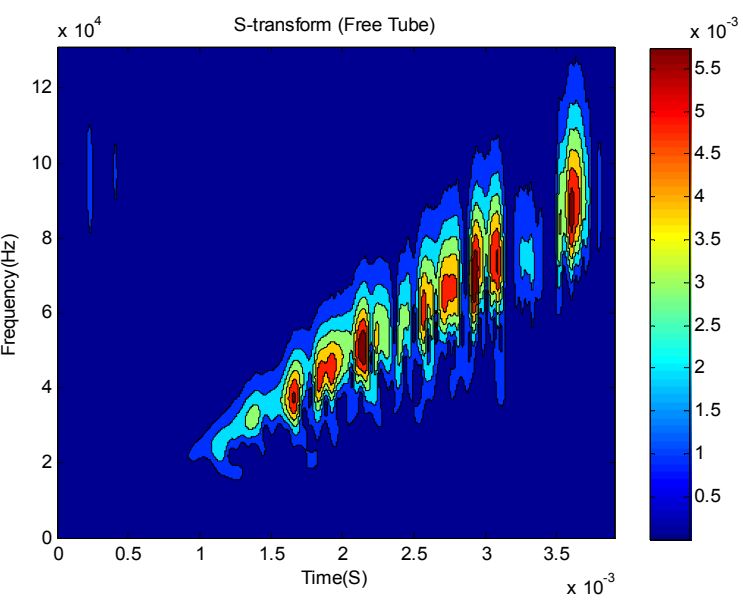

b) Clamped at the Left

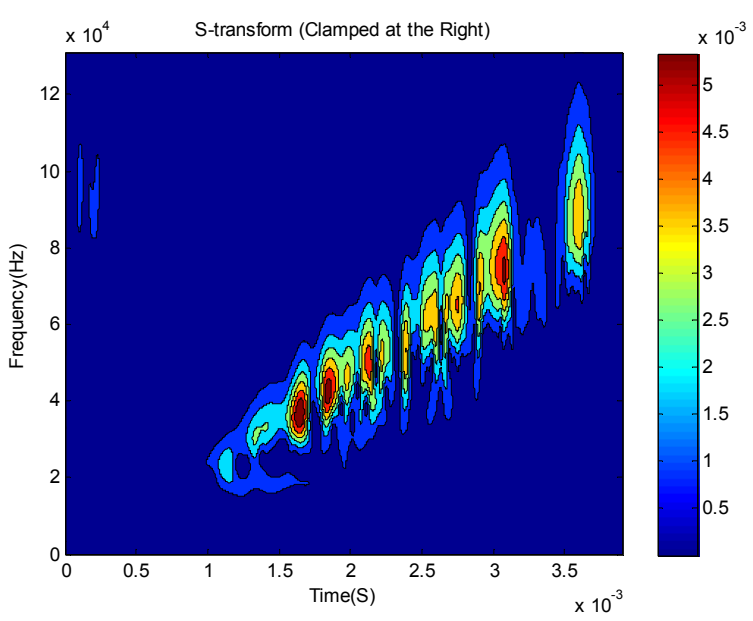

d) Clamped at the Right

Fig.73 S Transform of the Sensory Signals

Since the excitation signal was a sweep sine wave, the peaks located on a diagonal line correlated to the dominant frequency increasing with time. The amplitude level of the most significant peaks of the $\mathrm{S}$ transforms could be extracted to indicate the tube conditions. Fig.74 compares the characteristics of the peaks of the free tube and that of the tube clamped at the right. 


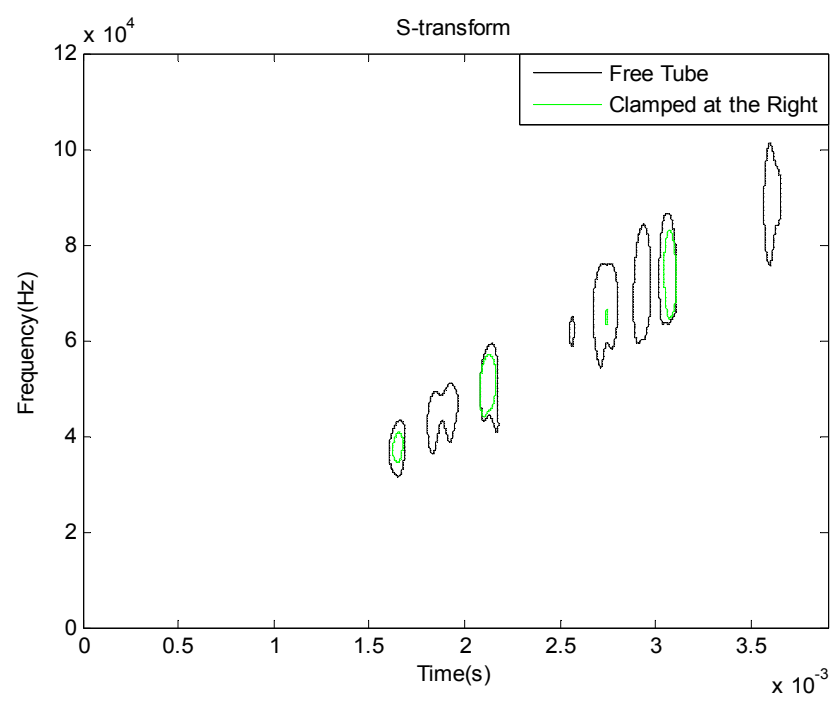

Fig.74 Contour of Peaks

The time, frequency and amplitude of the most significant peaks were isolated and utilized to estimate the conditions of tubes. Fig. 75 illustrates the peaks along the time axis and Fig.76 illustrates the peaks along the frequency axis.

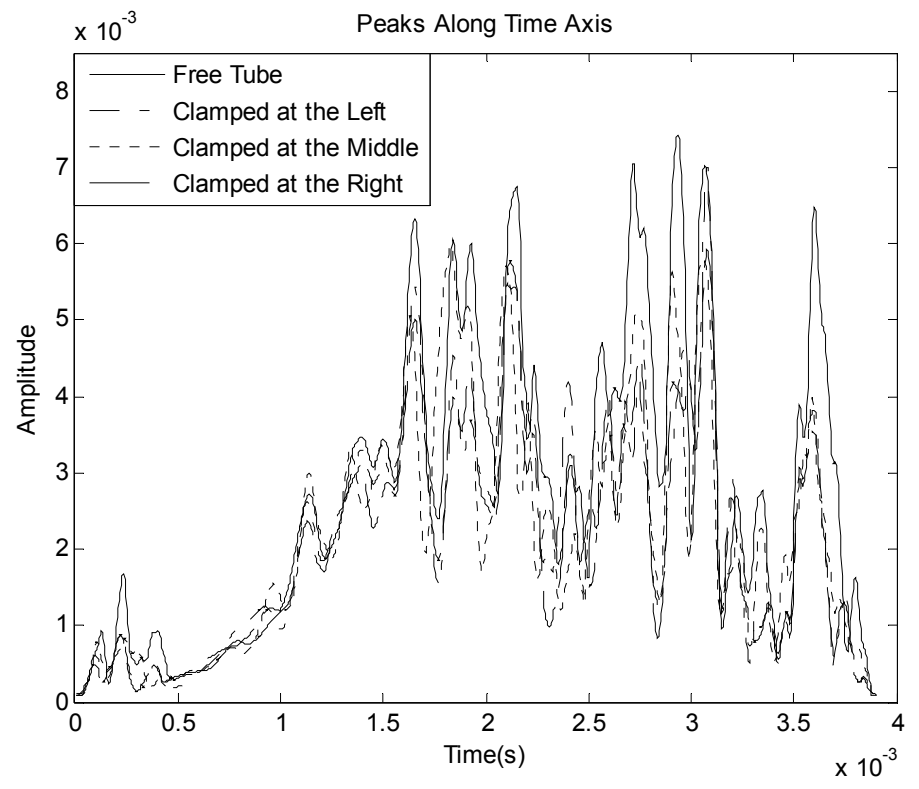

Fig.75 Maximum Amplitude Along Time Axis 


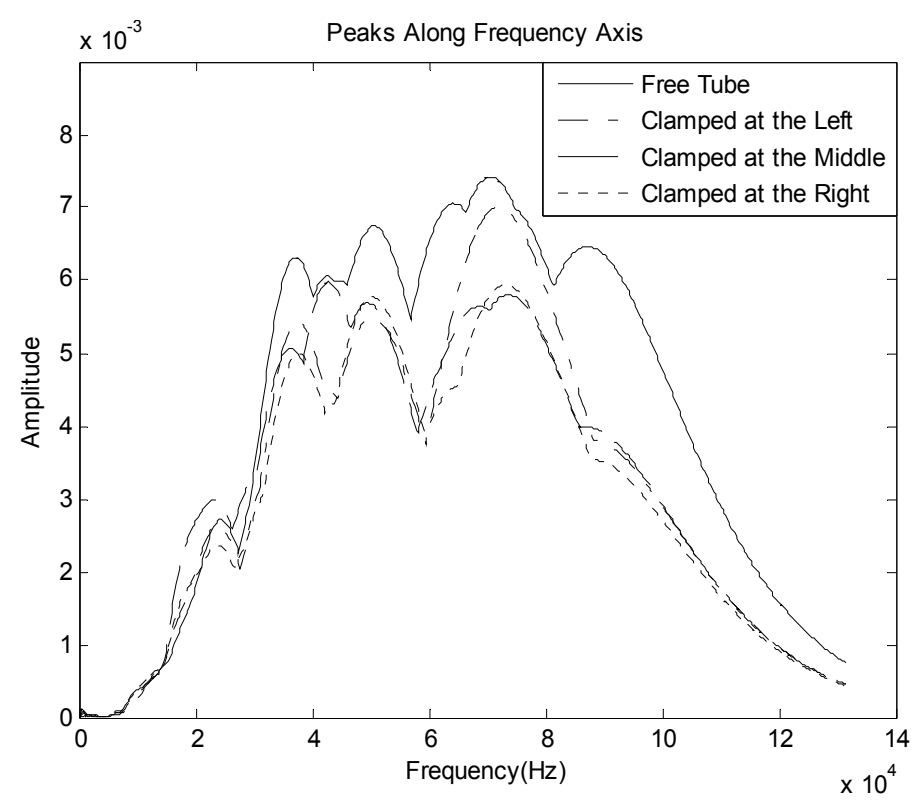

Fig.76 Maximum Amplitude Along Frequency Axis

The encoded information of time, frequency and amplitude extracted from peaks of $\mathrm{S}$ transforms of the dynamic responses under different conditions was presented to the backpropagation neural network for training. The inputs and the outputs of the backpropagation type neural network model are illustrated in Fig.77. The neural network had four inputs, four hidden nodes and one output. The output was a HI which represented the condition of the tube. The inputs were the encoded information of the frequency responses at four critical frequencies identified from the $\mathrm{S}$ transform plots. These frequencies were 37, 50, 70 and $87 \mathrm{KHz}$. From the experimental result, 16 cases were chosen for training while the other 16 cases were used for test. 


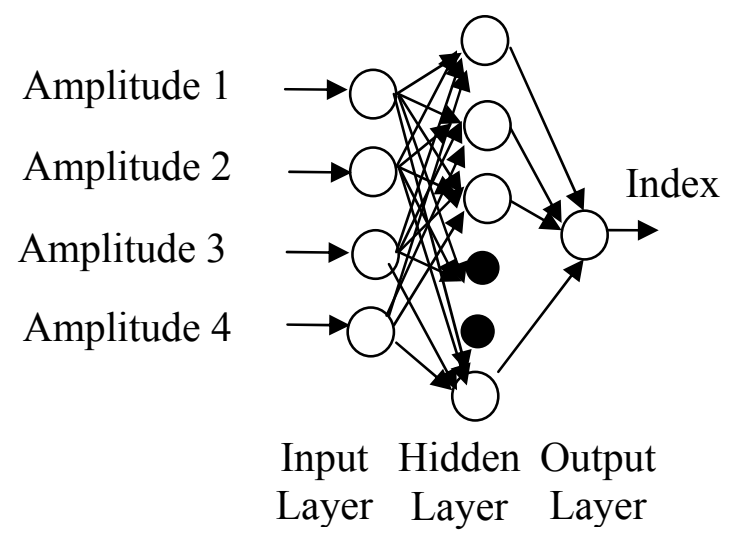

Fig.77 Backpropagation Neural Network Model

The training and the test were carried out for two different purposes. First, the diagnostic method aimed to separate the free and the clamped tubes. The HI of the training data extracted from the free tube's dynamic response was labeled as 0 , and all others were labeled as 1 . The training of the neural network converged quickly, and the estimation accuracy of the diagnostic system was $88 \%$. Out of the 16 test cases, only 2 cases were identified wrongly. Secondly, the location of the clamp was estimated by the neural network. The training and testing process was the same as the previous one except that the HIs of the training data was labeled as 2,3 or 4 according to the location of the clamp (left $=2$, middle $=3$, and right $=4$ ). After the training process, the neural network estimated $\mathrm{HI}$ of the tube with $38 \%$ accuracy for the test cases. Out of the 16 test cases, only 6 were classified correctly. Based on the results, the proposed method might be confidently used to determine the existence of a surface defects on a tube. If the clamp used for holding components on a tube loosen up, it would be possible to detect the problem. The confidence level would be much lower when the location of the obstruction is estimated. 


\subsubsection{Lamb Wave Based Monitoring}

Lamb wave based monitoring is an effective tool to evaluate defects on the surface of composite materials, since the propagation of Lamb wave is very sensitive to the variation of the material properties. In this study, experiments were carried out on the carbon fiber tubes to testify the proposed integration of IBR with Lamb wave based SHM. Fig. 78 shows the perfect tube and the defect tube. The defect tube has a notch cut in the middle and around the whole surface of the tube. The piezoelectric actuator attached on the tube was excited by three $300 \mathrm{KHz}$ pulses. A microprocessor Pic16F690 was programmed by Microchip's Pikit 2 to generate the pulses, then, the signal was amplified with an operational amplifier.

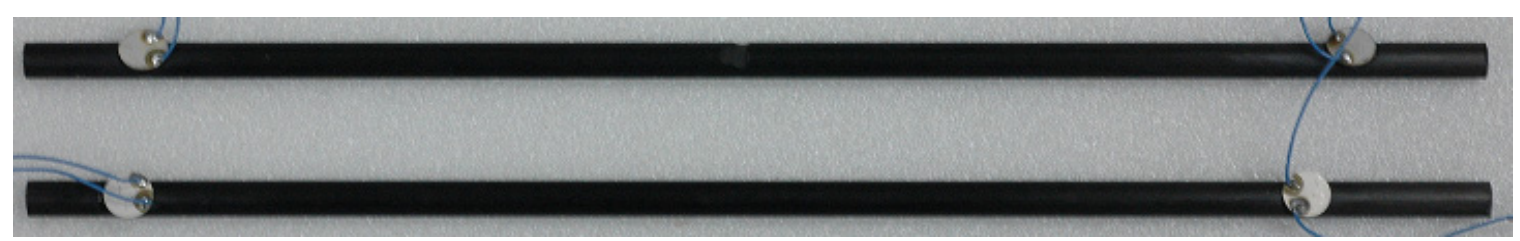

Fig.78 Perfect Tube and Defect Tube with a Notch

The generated Lamb waves would propagate along the surface of the tubes. Dynamic responses received by the piezoelectric sensor were acquired by a Nicolet Integra10 four channel digital oscilloscope. The experiment set up is shown in Fig.79. The same experimental procedure was repeated on the defect tube and on the perfect tube when it was squeezed at $40 \mathrm{~mm}, 80 \mathrm{~mm}$ or $120 \mathrm{~mm}$ away from the actuator. 


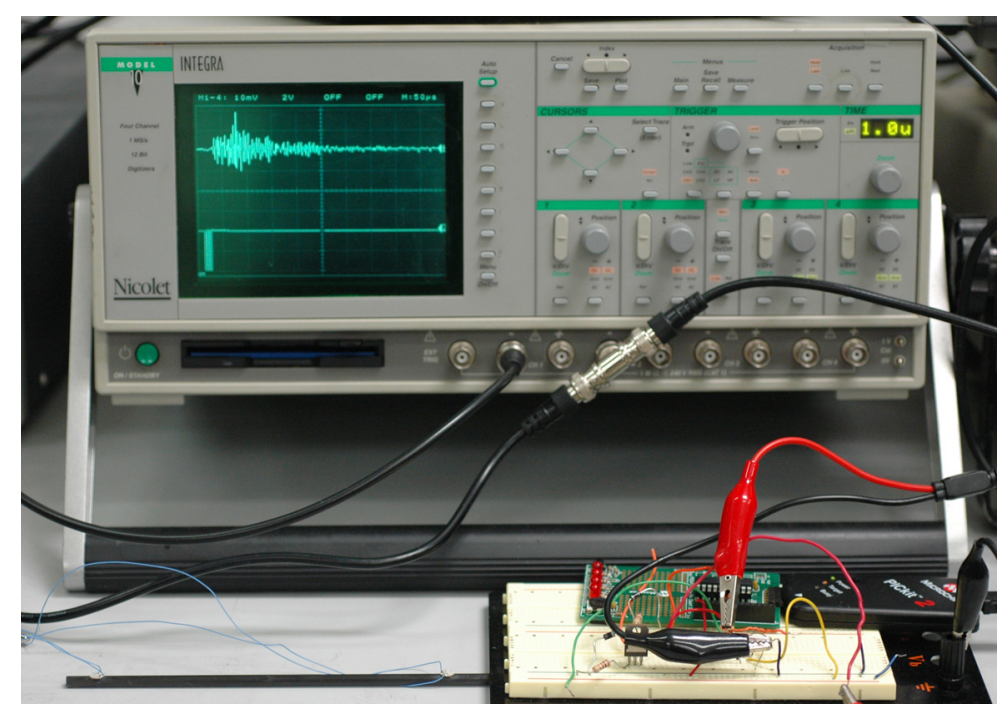

Fig.79 Experimental Setup for Lamb Wave Based Monitoring

The frequency response data collected by the piezoelectric sensor was processed by $\mathrm{S}$ transform to extract features. Fig. 80 shows the results of $\mathrm{S}$ transforms of the perfect tube, the perfect tube clamped at the left, at the middle, at the right and the defect tube.

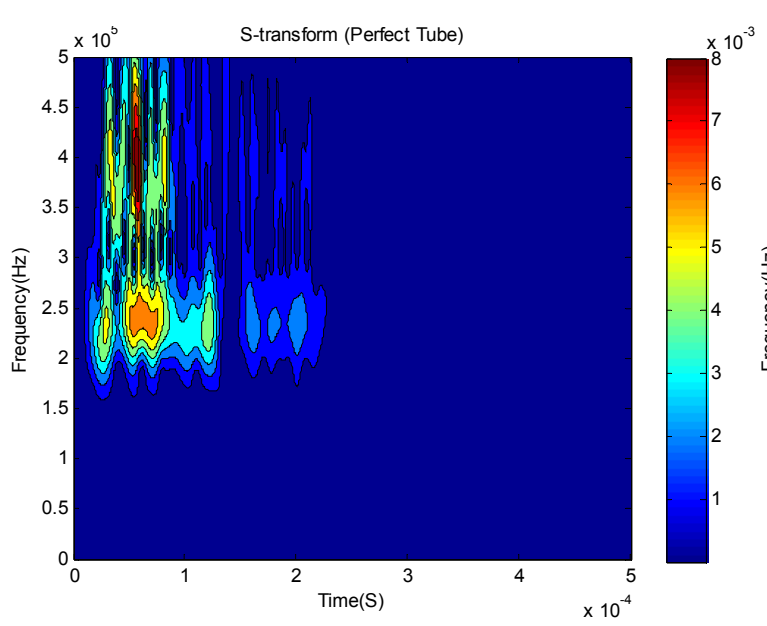

a) Perfect Tube

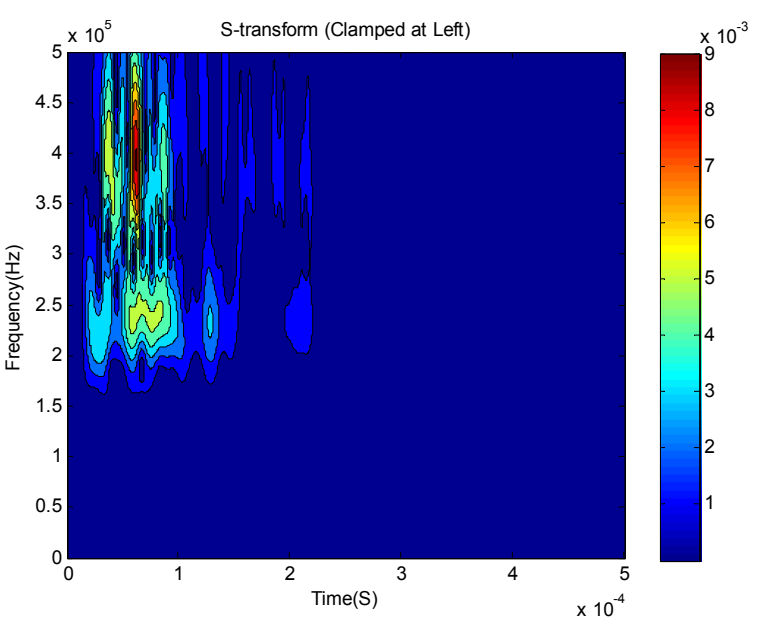

b) Perfect Tube Clamped at the Left

Fig.80 S Transform of Dynamic Responses of Perfect and Defect Tubes 


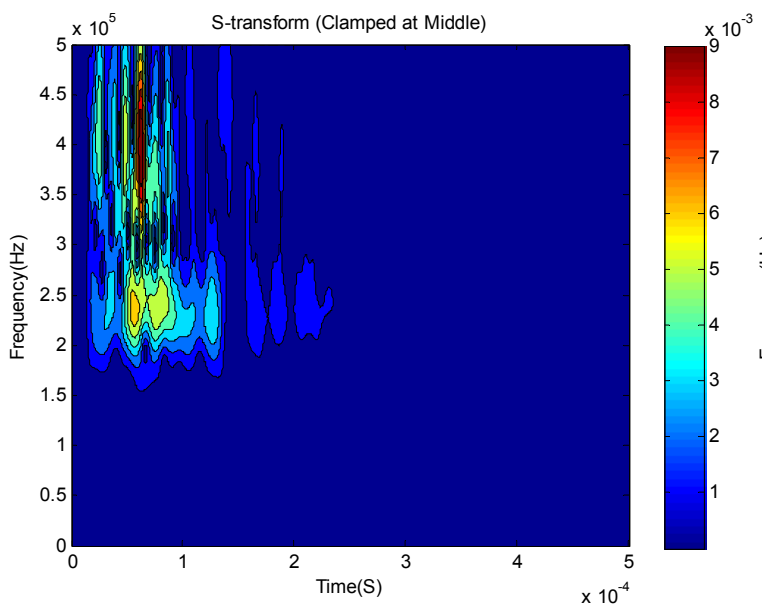

c) Perfect Tube Clamped at the Middle

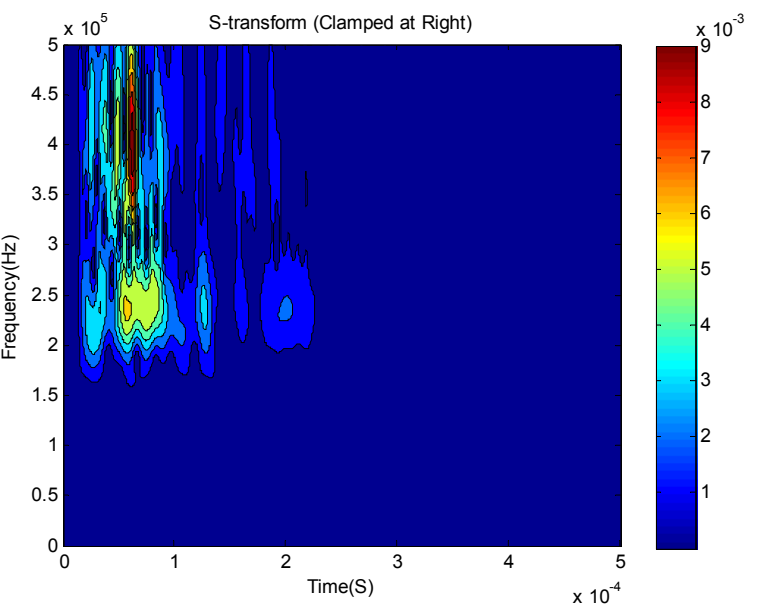

d) Perfect Tube Clamped at the Right

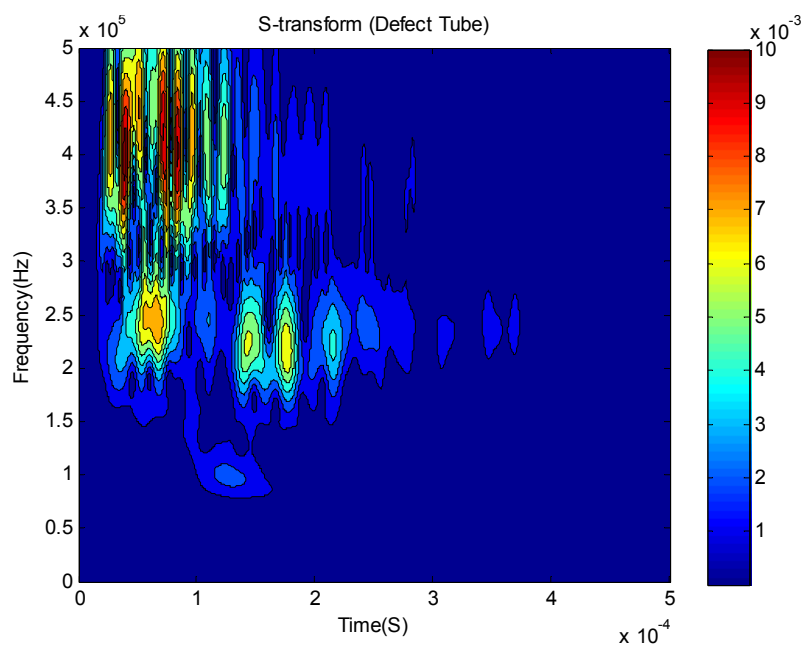

e) Defect Tube

From the figures, we can see that the peaks of S transforms occurred at different time, but they were almost at the same frequency. The frequency was about $250 \mathrm{KHz}$ which was the frequency of the pulse generated. The peaks of the $\mathrm{S}$ transforms indicated the receipt of the generated Lamb waves and its echo at the sensor side. From this we can estimate the delay time of the propagation of Lamb waves.

When we compared the results of the defect tube with the perfect tube, the noticeable difference was that, for the defect tube, there were two extra distinctive peaks, and the 
frequency of these peaks was lower than all other peaks. This might be the echo caused by the reflection when Lamb waves propagated to the notch. With further analysis of the occurred time of the peaks, the position of the defect might be identified.

\subsection{Experiments on Rotors}

For helicopters, propellers are among the most easily broken parts. When a propeller is damaged, its movement will be unbalanced and the shaft of the damaged propeller will be subjected to significant unbalanced centrifuge forces. In this study, IBR was applied to estimate the conditions of the propellers using FFT and a LUT.

The experiments carried out to determine the dynamic characteristics of the perfect and the damaged propellers were set up as presented in Fig.81. One of the arms carrying the four rotor helicopter was fixed on the Kistler 9279 Dynamometer. The electric motor rotated the propeller at $500 \mathrm{rpm}$, when $2 \mathrm{~V}$ DC was applied from a power supply. The frequency response of the arm was measured by the dynamometer and a piezoelectric strip attached to the tube. The spectrum analyzer collected and analyzed the sensory data from the dynamometer and the piezoelectric strip at the same time. The perfect propeller and the damaged propellers with $16.5 \mathrm{~mm}$ or $33 \mathrm{~mm}$ long missing tips were tested separately. The perfect and the damaged propellers are presented in Fig.82. The dynamometer measured the lift force and the torque when the propeller was perfect and damaged, which is shown in Fig.83. 


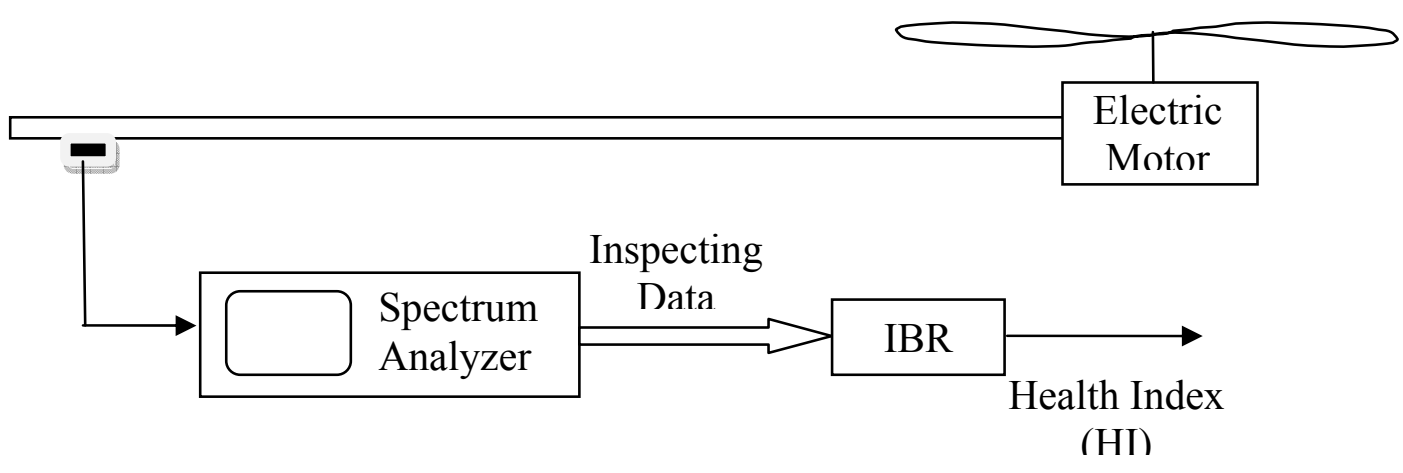

Fig.81 Proposed Procedure for the Inspection of the Propellers

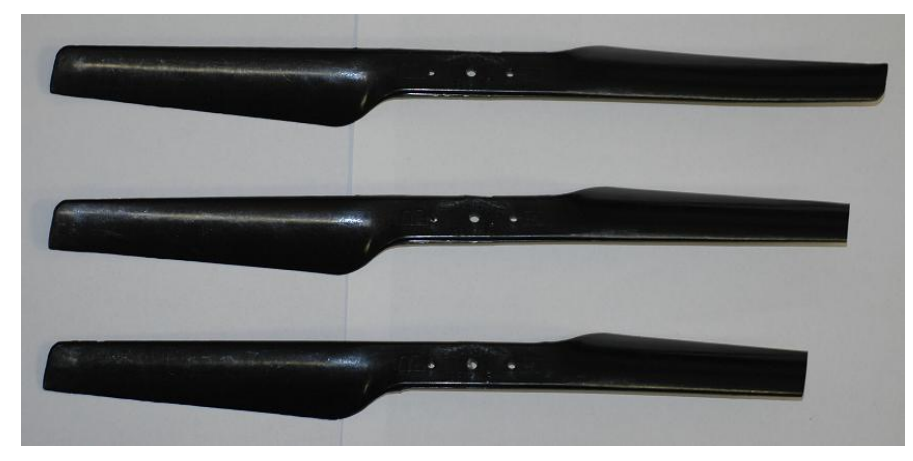

Fig.82 Perfect and Damaged Propellers

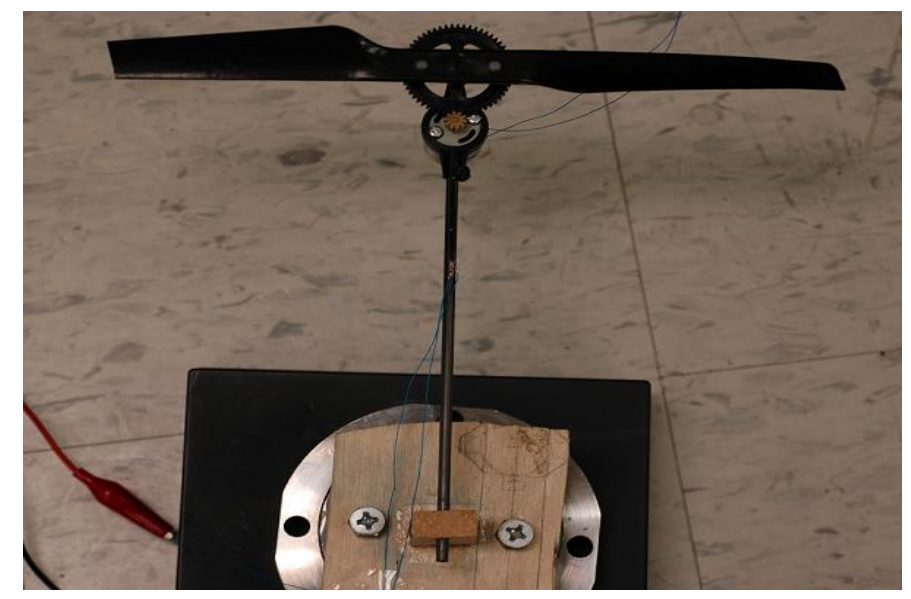

Fig.83 Experimental Setup to Measure the Frequency Responses of the Rotors

The dynamic responses of the perfect and the damaged propellers were measured when they were rotating. The experimental data was collected by digitizing the lift force 
of the dynamometer and the voltage coming from a piezoelectric strip. The FFT of the collected data from the dynamometer and the strip are presented in Fig.84 and Fig.85 respectively. The main indicator of the propeller damage was the relationship between the sharp spikes of the spectrums. The IBR classified the signals based on the characteristics of these spikes.

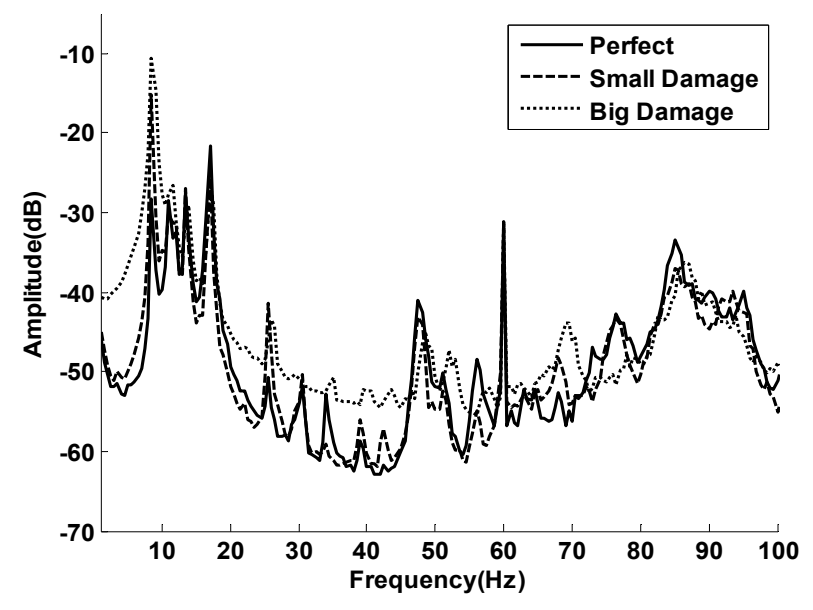

Fig.84 Frequency Responses of the Arms Measured by a Dynamometer

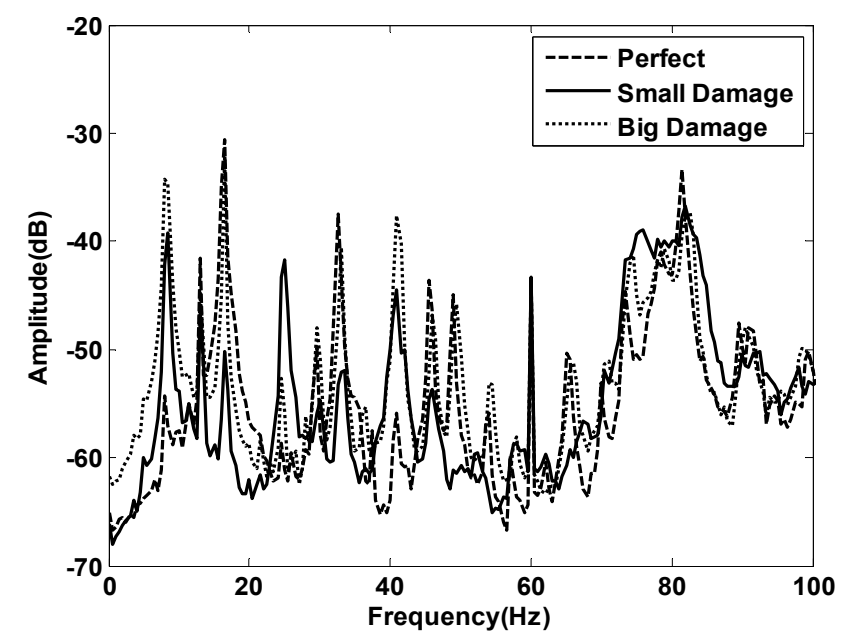

Fig.85 Frequency Characteristics of the Arms Measured by a Piezoelectric Strip

Fig.86 shows the IBR model to classify the perfect and the damaged propellers. The relationship among the heights of the dominant spikes, which are shown in Fig.84 was 
calculated to implement classification. From the FFT analysis, the frequencies and the amplitudes of the first two sharp spikes were extracted. Then the ratio of the amplitude of the first two peaks was calculated. If the ratio of the first peak to second peak is less than 1, it was classified as a perfect tube. The ratio quickly increased when one side of the propeller lost more material than the other side. The IBR distinguished the perfect and the damaged propellers from the characteristics of the spikes of beam vibration data in the frequency domain with no error in our tests.

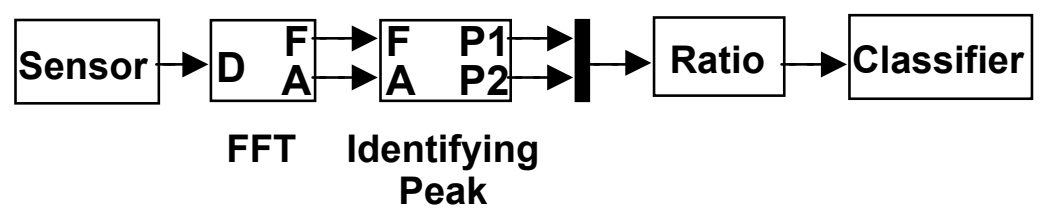

Fig.86 IBR Classification Model Based on the Ratio of the First Two Peaks

The arm branch of the helicopter was also been tested on the vibration test system. The sweep sine wave measurement was obtained by the piezoelectric disks. The piezoelectric actuators were excited by the sweep sine wave which had the range from 0 $\mathrm{Hz}$ to $100 \mathrm{~Hz}$. The piezoelectric sensors measured the dynamic response of the arm which included different combinations of the perfect tube, the damaged tubes, the perfect propeller, and the damaged propellers. The results were shown in Fig.87 a) and Fig.87 b). From the results, it was obvious that the dynamic response of the perfect tube and the perfect propeller had higher amplitude than others. If the average of the amplitude was calculated, it was easy to distinguish the perfect tube and the perfect propeller from others. However, it was difficult to distinguish among other combination of damaged tubes and propellers. 


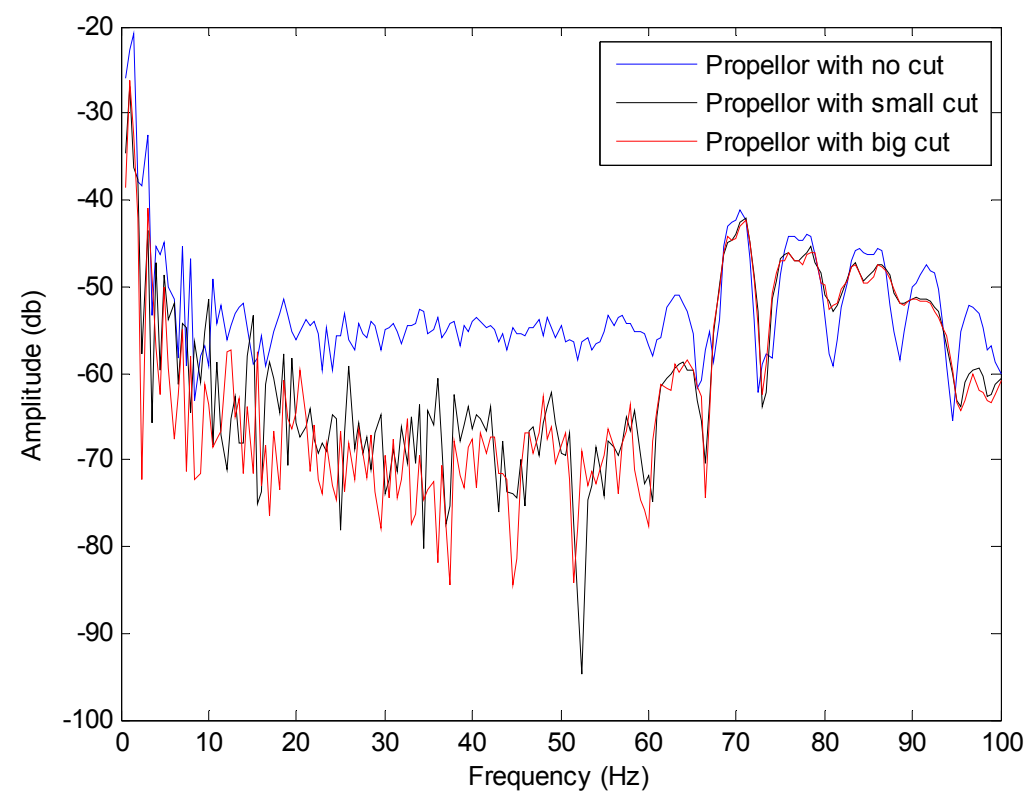

a) Perfect Tube with Different Propellers

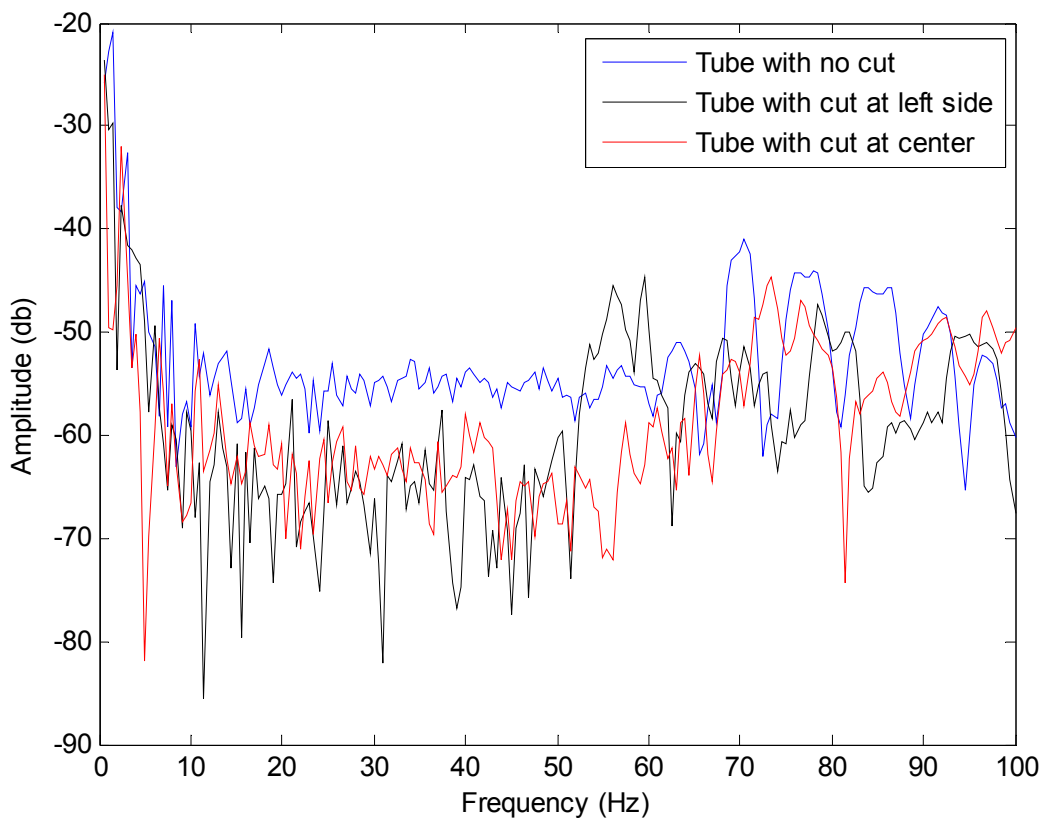

b) Perfect Propeller with Different Tubes

Fig.87 Frequency Responses of Different Combination of Tubes and Propellers 


\subsection{Conclusions}

In this study, IBR based SHM systems were developed and tested to evaluate the structural integrity of tubes and propellers of a four rotor helicopter. The perfect and the damaged carbon fiber composite tubes and propellers were excited using ambient vibrations, Lamb waves and sweep sine waves through the piezoelectric actuators bonded on their surface. Their frequency responses were measured by the piezoelectric sensors attached on the opposite side of the structures. The dominant frequency characteristics of the perfect and the damaged structural components were extracted with FFT and S transformation. They were found different and were used to estimate the integrity of these components.

The experimental study of composite tubes indicated that the defects may be identified by evaluating the frequency response characteristics of a structure. The size, location, and direction of the defect affected the frequency response characteristics of the structure. A defect located at the fixed end of a tube and was parallel to the measurement direction was easier to identify comparing to the case when the same defect was located at the free end. The spectral responses of the perfect and the damaged tubes changed very little when the hole was perpendicular to the direction of the measurement, no matter if they were close to the fixed end or not. These results were very reasonable since the stress was concentrated on the front and back of the tube. A hole which is perpendicular to the measured vibration direction would influence the stress concentrated region much less than a hole parallel to the measured vibration direction of the tube.

The Lamb wave approach is more suitable to identify the weakened structural elements for maintenance purposes. It is very sensitive to the property change of the 
structure surface, and it can be used to identify the location of damages. However, the Lamb wave approach requires more sensors for the large size assemblies, since at least one sensor and actuator are required for every structural element. Another disadvantage of this method is that special equipment is needed to generate Lamb waves on the structures' surface, which makes it difficult to apply Lamb waves on board as part of SHM system.

In the experimental studies, piezoelectric elements provided excellent signals to identify dynamic characteristics of the structures. If a structure was excited by ambient vibrations, only one piece of piezoelectric element was needed to identify the structural integrity. The amplitude of sensory data was good enough to be input directly to the oscilloscope without any conditioning.

The dominant frequency characteristics could be extracted and encoded through transformation algorithms. S transformation not only can get the FFT analysis but it can also provide time frequency representation. It was a perfect match to analyze signals acquired through Lamb wave approach. The frequency features were then presented to neural networks for training. The training results were simplified as LUTs for IBR to classify the structural integrity with HI values. The proposed approach was computational efficient, and it could be implemented by microprocessors. The classification results of IBR were acceptable accurate. 


\section{CHAPTER VII}

\section{CONCLUSIONS AND FUTURE WORK}

In the previous chapters, the Index Based Reasoning (IBR) system was introduced and feasibility was studied. In this section, the important findings of the study and the possible future work are presented.

\subsection{Conclusions}

The IBR was developed as a small low cost distributed diagnostic system for small UAVs. Later, various capabilities were added to make it effective even in more demanding conditions and to satisfy the needs of bigger UAVs. The proposed IBR was used to identify the structural integrity of complex systems based on the dynamic responses of ambient vibration, Lamb wave and sweep sine wave signals. Simulation and experimental works proved that IBR systems could be applied to a wide range of systems to evaluate the health conditions of versatile structures.

The listed conclusions of this chapter were based on the simulation and experimental work:

- 3D graphic user interface was developed to program or train individual IBR nodes. The IBR node could be trained to learn a pattern presented to it. An IBR network with two layer structure was prepared. It processed the information of both time and frequency domain. The programmer could automatically generate the rule based MicroBasic code for the PIC processors of Microchip Corporation.

- The models of a High Displacement Actuator (HDA) and a turbojet engine SR30 were simulated. The IBR systems used the temperature and the frequency response of the simulated system to monitor the health conditions. It was easy to 
- The simulation of a four rotor helicopter and its control system was demonstrated. The PID controller and the fuzzy logic controller were designed to guide the four rotor helicopter climbing to the target height and hovering around. The controllers were integrated with IBR to optimize the control strategy based on the structural integrity. Fuzzy logic controller was more flexible; it could provide fault tolerant control for UAVs. However, it needed prior knowledge and a tedious trial and test process to design the membership functions and the rule set.

- Experimental works were carried out on the components of the four rotor helicopter; the carbon fiber tubes and the propellers. The piezoelectric elements were utilized as actuators and sensors; they were light and thin, which could be attached on the structures easily. Their signals could be directly acquired with A/D converting. Simple amplifying circuits might be needed to amplify the signals; however, for most of the cases, their signals were strong enough to be utilized directly. They also worked well at low frequency and could monitor the strain.

- The structures were excited by the ambient vibration, the Lamb wave and the sweep sine wave signals. The health monitoring systems based on the Lamb wave or the sweep sine wave needed at least two piezoelectric elements for one structure, and they generated more descriptive frequency domain characteristics. 
However, they needed external equipment and power to generate the Lamb wave and the sweep sine wave, and they needed high frequency sampling data acquisition systems. Lamb wave propagations were found sensitive to surface damages, and they could be analyzed to identify the location of the problems. The health monitoring based on ambient vibration needed less sensors, less power, and less equipment. It might be used to identify global changes according to the occurrence of damages, but it could not provide much local information, such as the location of the damage.

- Simulation and experimental works showed that frequency response characteristics of the structures could be used to detect significant defects. If a structure operated as a cantilever beam, then, the size, the location and the orientation of the damage would affect the characteristics of the frequency responses. The frequency of the dominant mode changed when the structure weakened. It decreased with the increase of the distance between the damage and the fixed end. However, it was much easier to identify the shift if a through hole was located at the fixed end of a tube and was parallel to the measurement direction. Identification of the hole was much more difficult when it was at the same location but perpendicular to the measurement direction.

- The proposed IBR approach was found computationally efficient and easy to implement for experimental data. FFT and S transformation were utilized to extract frequency and time-frequency characteristics of the data respectively. $\mathrm{S}$ transformation was found more accurate to obtain the time-frequency 
representation of the signals. $\mathrm{S}$ transformation results might be encoded to interpret the signal by using various methods.

- Self organizing maps (SOM) were also used for the preparation of the IBRs. The learned patterns were used for selection of the parameters of the IBRs. The proposed approach was found suitable for applications with lack of prior knowledge about the characteristics of the system, which is the case for most of the real life problems. The proposed approach reduced the computation burden, thus, it could be implemented by simple and cheap hardware.

- The IBR evaluated and classified the health of the sub-system with an index. It was carried to the higher level IBRs layer by layer. A wide range of methods could be conveniently embedded into IBR nodes to improve the performance of feature extraction and decision making. The IBR may be easily adopted to be used for classifying the data of many applications.

- The preliminary hardware implementation of the IBR was prepared by using PIC microcontrollers. Multiple versions were prepared to accept Analog or Digital inputs and to report the condition with either analog (PWM) or digital signals.

\subsection{Future Work}

The simulation and experimental works proved that IBR systems are efficient, flexible and economic for small UAVs. However, further research is needed for commercialization of the proposed concept in this thesis:

1. Extensive testing of the frequency domain characteristics of the structure. The four rotor helicopter studied in this thesis will be replaced with new models soon. 
2. Development of automatic programming code for IBRs with three inputs when they use the look up tables. The automatic programming capability was developed only for the rule based IBR in the thesis.

3. Consideration of the faster algorithms such as Kalman filters to add more efficient signal processing capability to IBRs.

4. Implementation of DSPs for development of faster and more advanced SHM systems. 


\section{LIST OF REFERENCES}

Aamodt, A., and E. Plaza, "Case-Based Reasoning: Foundational Issues, Methodological Variation and System Approaches," Al Communications, Amsterdam: IOS Press, 1994, pp. 39-59.

Abraham, A., "Rule-based Expert Systems," Handbook of Measuring System Design, Edited by Peter H. Sydenham and Richard Thorn, 2005, John Wiley \& Sons, pp. 909-919.

Alampalli, S., G. Fu, and E. W. Dillon, "Signal Versus Noise in Damage Detection by Experimental Modal," Journal of Structural Engineering, 1997, Vol. 123, No. 12, pp. $237-245$

Altuğ, E., J. P. Ostrowski, and C. J. Taylor, "Control of a Quad-rotor Helicopter Using Dual Camera Visual Feedback," International Journal of Robotics Research, Vol. 24, No. 5, 2005, pp. 329-341.

Araki, M., and H. Taguchi, "Two-Degree-of-Freedom PID Controllers," International Journal of Control, Automation, and Systems, Vol. 1, No. 4, 2003, pp. 401-411.

Banks, H. T., D. J. Inman, D. J. Leo and Y. Wang, "An Experimentally Validated Damage Detection Theory in Smart Structures," Journal of Sound and Vibration, Vol. 191, No. 5, 1996, pp. 859-880.

Bayraktara, E., S. D. Antolovichc, and C. Bathiasb, "New Developments in Nondestructive Controls of the Composite Materials and Applications in Manufacturing Engineering," Journal of Materials Processing Technology, Vol. 206, No. 1-3, 2008, pp. 30-44. 
Belkic, D., P. A. Dando, J. Main, H. S. Taylor, and S. K. Shin, "Decimated Signal Diagonalization for Fourier Transform Spectroscopy," J. Phys. Chem. A 2000, Vol. 104, pp. 11677-11684.

Bickraj, K., T. Pamphile, A. Yenilmez, M. Li, and I. N. Tansel, "Fuzzy Logic Based Integrated Controller for Unmanned Aerial Vehicles," Florida Conference on Recent Advances in Robotics (FCRAR 2006), Miami, 2006.

Biggs, R. E., "Space Shuttle Main Engine, The First Ten Years," History of Liquid Rocket Engine Development in the United States, 1955-1980, American Astronautical Society History Series, Vol. 13, Part 3, Chapter 4, 1992, pp. 69122.

Buchanan, B. G., and R. O. Duda, "Principles of Rule-Based Expert Systems," Technical Report, STAN-CS-TR-82-926, Stanford University, 1982.

Carden, E. P. and P. Fanning, "Vibration Based Condition Monitoring: A Review," Structure Health Monitoring, Vol.3, No.4, 2004, pp. 355-377.

Castillo, P., A. Dzul, and R. Lozano, "Real-time Stabilization and Tracking of a Four-rotor Mini Rotorcraft," IEEE Transaction on Control Systems Technology, Vol. 12, No. 4, 2004, pp. 510-516.

Cawley, P., and R. D. Adams, "The Location of Defects in Structures from Measurements of Natural Frequencies," Journal of Strain Analysis, Vol. 14, No. 2, 1979, pp. 49-57. 
Cawley, P., and R. Ray, "A Comparison of the Natural Frequency Changes Produced by Cracks and Slots," ASME Journal of Vibration, Acoustics, Stress and Reliability in Design, 1998, pp. 366-370.

Chandramohan, A., M. V. C. Rao, and M. S. Arumugam, "Two New and Useful Defuzzification Methods Based on root Mean Square Value," Soft Computing, Vol. 10, 2006, pp. 1047-1059.

Chou, J. H., and J. Ghaboussi, "Genetic Algorithm in Structural Damage Detection," Computers and Structures, Vol. 79, 2001, pp. 1335-1353.

Cooley, J., P. Lewis, and P. Welch, "The Finite Fourier Transform," IEEE Transactions on Audio Electroacoustics, Vol. 17, No. 2, 1969, pp. 77-85.

Cottrell, M., "Advances in Self-Organizing Maps," Neural Networks, Vol. 19, 2006, pp. 721-722.

Degertekin, F. L., and B. T. Khuri-Yakub, "Lamb Wave Excitation by Hertzian Contacts with Applications in NDE," IEEE Transactions on Ultrasonics, Ferroelectrics, and Frequency Control, Vol. 44, No. 4, 1997, pp. 769-777.

Devaney, M., B. Cheetham, "Case-Based Reasoning for Gas Turbine Diagnostics," In 18th International FLAIRS Conference (FLAIRS-05), American Association for Artificial Intelligence (www.aaai.org), 2005.

Diamanti, K., J. M. Hodgkinson, and C. Soutis, "Detection of Low-velocity Impact Damage in Composite Plates Using Lamb Waves," Structural Health Monitoring, Vol. 3, No. 1, 2004, pp. 33-41. 
Dilena, M., and A. Morassi, "Structural Health Monitoring of Rods Based on Natural Frequency and Antiresonant Frequency Measurements," Structural Health Monitoring, Vol. 8, No. 2, 2009, pp. 149-173.

Ding, Y. L., and A. Q. Li, "Structural Health Monitoring of Long-span Suspension Bridges Using Wavelet Packet Analysis," Earthquake Engineering and Engineering Vibration, Vol. 6, No. 3, 2007, pp. 289-294.

DImasry, M. I. S., and E. A. Johnson, "Health Monitoring of Structures Under Ambient Vibrations using Semiactive Devices," Proceeding of the 2004 American Control Conference, Boston, Massachusetts, 2004, pp. 3526-3531.

Duhamel, P., and M. Vetterli, "Fast Fourier Transforms: A Tutorial Review and a State of the Art," Signal Processing, Vol. 19, 1990, pp. 259-299.

Erginer, B., and E. Altug, "Modeling and PD Control of a Quadrotor VTOL Vehicle," Proceedings of the 2007 IEEE Intelligent Vehicles Symposium, Istanbul, Turkey, June 13-15, 2007.

Figueroa, F., R. Holland, J. Schmalzel, and D. Duncavage, “Integrated System Health Management (ISHM): Systematic Capability Implementation," SAS 2006- IEEE Sensors Applications Symposium, Houston, Texas, USA, 2006, pp. 199-205.

Figueroa, F., J. Morris, D. Nickles, J. Schmalzel, D. Rauth, A. Mahajan, L. Utterbach, and C. Oesch, "Intelligent Sensors and Components for On-Board ISHM," 42nd AIAA/ASME/SAE/ASEE Joint Propulsion Conference \& Exhibit, Sacramento, California, July 9 - 12, 2006. 
Filipic, B., and M. Junkar, "Using Inductive Machine Learning to Support Decision Making in Machining Processes," Computers in Industry, Vol. 43, 2000, pp. 3141.

Freudenthaler, B., G. Gutenbrunner, R. Stumptner, and J. Küng, "Case-based Decision Support for Bridge Monitoring," The Third International MultiConference on Computing in the Global Information Technology, 2008, pp. 3137.

Fritzen, C. P., "Vibration-based Structural Health Monitoring - Concepts and Applications," Key Engineering Materials, Vol. 293-294, 2005, pp.3-20.

Garg D. P., M. A. Zikry, and G. L. Anderson, "Current and Potential Future Research Activities in Adaptive Structures: an ARO Perspective," Smart Materials and Structures, No.10, 2001, pp. 610-623.

Georgin, E., F. Bordin, J. R. McDonald, "Using Prototypes in Case Based Diagnosis of Steam Turbines," Proceedings of IEE Case Based Reasoning: Prospects for Applications, 1995.

Hamel, T., R. Mahony, R. Lozano, and J. Ostrowski, "Dynamic Modeling and Configuration Stabilization for an X4-flyer," Proceedings of the International Federation of Automatic Control Symposium (IFAC 2002), Barcelona, Spain, 2002.

Hurlebaus, S., M. Niethammer, L. J. Jacobs and C. Valle, "Automated Methodology to Locate Notches with Lamb Waves," Acoustical Society of America, Vol. 2, No. 4, 2001, pp. 97-102. 
Jassbi, J. J., P. J. A. Serra, R. A. Ribeiro, and A. Donati, "A Comparison of Mamdani and Sugeno Inference Systems for a Space Fault Detection Application," Automation Congress, WAC, 2006.

Kaphle, M. R., A. C. C. Tan, and D. Thambiratnam, "Structural Health Monitoring of Bridges Using Acoustic Emission Technology and Signal Processing Techniques," 13th Asia Pacific Vibration Conference, 2009, Christchurch, New Zealand.

Ke, M., and M. All, "MLS, A Machine Learning System for Engine Fault Diagnosis," Proceedings of the 1st International Conference on Industrial and Engineering Applications of Artificial Intelligence and Expert Systems, Vol. 2, 1988, Tullahoma, Tennessee, United States, pp. 712-727.

Kessler, S. S., S. M. Spearing, and C. Soutis, "Damage Detection in Composite Materials using Lamb Wave Methods," Proceedings of the American Society for Composites, 9-12 September, 2001, Blacksburg, VA.

Ko, J. M., and Y. Q. Ni, "Structural Health Monitoring and Intelligent Vibration Control of Cable-supported Bridges: Research and Application," KSCE Journal of Civil Engineering, Vol. 7, No. 6, 2003, pp. 701-716.

Kohonen, T, "Self-Organizing Maps. Series in Information Sciences," Vol. 30, Springer, Heidelberg, Second ed, 1997.

Kohonen, T., "Self-organizing Maps," Third Edition, Springer-Verlag Berlin Heidelberg New York, 2001.

Konstantinidis, G., P. D. Wilcox, and B. W. Drinkwater, "An Investigation into the Temperature Stability of a Guided Wave structural Health Monitoring System 
Using Permanently attached Sensors," IEEE Sensors Journal, Vol. 7, No. 5, May 2007, pp. 905-912.

Kundu, S., "The Min-max Composition Rule and its Superiority over the Usual Max-min Composition Rule," Fuzzy Sets and Systems, Vol. 93, 1998, pp. 319329.

Lamb, H., "On Waves in an Elastic Plate," Proceedings of the Royal Society of London, Series A, Containing Papers of a Mathematical and Physical Character, Vol. 93, No. 648, 1917, pp. 114-128.

Lee, M. R., "An Exception Handling of Rule-Based Reasoning Using Case-Based Reasoning," Journal of Intelligent and Robotic Systems, Vol. 35, 2002, pp. 327338.

Legendre, S., D. Massicotte, J. Goyette and T. K. Bose, "Wavelet-TransformBased Method of Analysis for Lamb-Wave Ultrasonic NDE Signals," IEEE Transactions on Instrumentation and Measurement, Vol. 49, No. 3, 2000, pp. 524-530.

Lei, Y. J. and B. S. Wang, "Design of an Aircraft Auto-landing Fuzzy Controller Based on ANFIS," Journal of System Simulation, 16, 2004, pp. 2580-2583.

Li, C. L., C. C. Yang, and C. J. Wu, "Time-optimal Control of a Hovering Quadrotor Helicopter," Journal of Intelligent and Robotic Systems, Vol. 45, 2006, pp. $115-135$.

Li, M., M. Demetgul, X. H. Li, and I. Tansel, "Integrated System Health Management by Using the Index Based Reasoning (IBR) and Self Organizing Map (SOM) Combination," 4th International Conference on Recent Advances in Space Technologies (RAST), 2009, Turkey, pp. 181-185. 
Li, M., M. Demetgul, I. Tansel, "Single Chip Index Based Reasoner (SCIBR) for Small Satellites," International Workshop on Small Satellites, New Missions and New Technologies, June 2008, Turkey.

Li, T. S., and C. L. Huang, "Defect Spatial Pattern Recognition using a Hybrid SOM-SVM Approach in Semiconductor Manufacturing," Expert Systems with Applications, Vol. 36, No. 1, 2009, pp. 374-385.

Loewke, K., D. Meyer, A. Starr, and S. Nemat-Nasser, "Structural Health Monitoring Using FFT," Smart Structures and Materials 2005: Sensors and Smart Structures Technologies for Civil, Mechanical and Aerospace Systems, Proceedings of SPIE, Vol. 5765, pp. 931-935.

Loh, C. H., and Z. K. Lee, "Substructural Identification of a Bridge-A FFT-Based Spectral Analysis," Structural Health Monitoring: Current Status and Perspectives, 1997, pp. 111-121.

Lopes, V., G. Park, H. H. Cudney, and D. J. Inman, "Impedance-Based Structural Health Monitoring with Artificial Neural Networks," Journal of Intelligent Material Systems and Structures, Vol.11, 2000, pp. 206-213.

Lu, Y., X. Wang, J. Tang and Y. Ding, "Damage Detection Using Piezoelectric Transducers and the Lamb Wave Approach: II. Robust and Quantitative Decision Making," Smart Materials and Structures, No. 7, 2008, pp.1-13.

Luangvilai, K., W. Punurai, and L. J. Jacobs, "Guided Lamb Wave Propagation in Composite Plate/Concrete Component," Journal of Engineering Mechanics, Vol. 128, No. 12, 2002, pp. 1337-1341. 
Masuoka, R., N. Watanabe, A. Kawamura, Y. Owada, and K. Asakawa, "Neurofuzzy System-Fuzzy Inference Using a Structure Neural Network," Proceedings of the International Conference on Fuzzy Logic \& Neural Networks, Lizuka, Japan, 1990, pp. 173-177.

Mckerrow, P., "Modelling the Draganflyer Four-rotor Helicopter," Proceeding of the 2004 IEEE International Conference on Robotics \& Automation, New Orleans, LA, April, 2004 .

Mrad, R. B., A. Abhari, and J. Zu, "Control Strategies for an Inchworm Piezomotor," International Symposium on Computational Intelligence for Measurement Systems and Applications, CIMSA2003, Lugano, Switzerland, July, 2003.

Mujica, L.E., J. Vehı', W. Staszewski, and K. Worden, "Impact Damage Detection in Aircraft Composites Using Knowledge-based Reasoning," Structural Health Monitoring, 2008, Vol. 7, No. 3, pp. 215-230.

Niethammer, M., L. J. Jacobs, J. M. Qu and J. Jarzynski, "Time-frequency Representations of Lamb Waves," Acoustical Society of America, Vol. 109, No.5, 2001, pp. 1841-1847.

Nurnberger, A., and D. N. R. Kruse, "Neuro-fuzzy Control Based on the NEFCON-Model: Recent Developments," Soft Computing, Vol. 2, 1999, pp. 168182.

Olsson, E., P. Funk, and N. Xiong, "Fault Diagnosis in Industry Using Sensor Readings

and Case-Based Reasoning," Journal of Intelligent \& Fuzzy Systems, Vol. 15, 2004, pp. 41-46. 
Osbomr, G. H., R. Gordon, H. L. Coplen, and G. S. James, "Liquid-hydrogen Rocket Engine Development at Aerojet, 1944 - 1950," Pennsylvania State Univ., NASA Propulsion Engineering Research Center, Vol. 2, 1997, pp.38-44.

Pakrashi, V., and B. Ghosh, "Application of S Transform in Structural Health Monitoring," NDTCE'09, Non-Destructive Testing in Civil Engineering, 2009, Nantes, France.

Paliwal, K. K., and L. D. Alsteris, "On the Usefulness of STFT Phase Spectrum in Human Listening Tests," Speech Communication, Vol. 45, No. 2, 2005, pp. 153170.

Patterson-Hine, A., G. Aaseng, G. Biswas, S. Narasimhan, and K. Pattipati, "A Review of Diagnostic Techniques for ISHM Applications," 1st Integrated Systems Health Engineering and Management Forum, Napa, CA, November, 2005.

Patterson-Hine, A., W. Hindson, D. Sanderfer, S. Deb, and C. Domagala, "A Model-based Health Monitoring and Diagnostic System for the UH-60 Helicopter," Presented at the American Helicopter Society $57^{\text {th }}$ Annual Forum, Washington, DC, May 9-11, 2001.

Pawar, P. M., and S. N. Jung, "Support Vector Machine Based Online Composite Helicopter Rotor Blade Damage Detection System," Journal of Intelligent Material Systems and Structures, Vol. 19, No. 10, 2008, pp. 1217-1228.

Pei, J., M. I. Yousuf, F. L. Degertekin, B. V. Honein, and B. T. Khuri-Yakub, "Lamb Wave Tomography and its Application in Pipe Erosion/Corrosion Monitoring," IEEE Ultrasonics Symposium, 1995, pp. 795-798. 
Penn, B. S., "Using Self-organizing Maps to Visualize High-dimensional Data," Computers \& Geosciences, Vol. 31, No. 5, 2005, pp. 531-544.

Poll, S., and D. Iverson, "Characterization of Model-based Reasoning Strategies for Use in IVHM Architectures," Proceedings of the SPIE, 2003, Vol. 5107, pp. 94 $-105$.

Pozzi, M., and T. King, "Piezoelectric Modelling for an Impact Actuator," Mechatronics, Vol. 13, 2003, pp. 553-570.

Querry, S., http://www.polyvionics.com, 2006.

Reda Taha, M. M., A. Noureldin, J. L. Lucero, and T. J. Baca, "Wavelet Transform for Structural Health Monitoring: A Compendium of Uses and Features," Structural Health Monitoring, 2006, Vol. 5, No.3, pp. 267-295.

Rose, J. L, "Guided Wave Nuances for Ultrasonic Nondestructive Evaluation," IEEE Transactions on Ultrasonics, Ferroelectrics, and Frequency Control, Vol. 47 , No. 3, 2000 , pp. 575-583.

Rumelhart, D. E., G. E. Hinton, and R. J.Williams, "Learning Internal Representations by Error Propagation," Parallel Distributed Processing: Explorations in the Microstructure of Cognition, Chapter 8, 1986, pp. 318-361, MIT Press.

Salawu, O. S., "Detection of Structural Damage through Changes in Frequency: A Review," Engineering Structures, Vol. 19, No. 9, 1997, pp. 718-723. 
Schein, J., and S. T. Bushby, "A Hierarchical Rule-Based Fault Detection and Diagnostic Method for HVAC Systems," HVAC\&R Research, Vol. 12, No. 1, 2006, pp. 111-127.

Stieler, F., H. Yan, F. Lohr, F. Wenz, and F. F. Yin, "Development of a Neurofuzzy Technique for Automated Parameter Optimization of Inverse Treatment Planning," Radiation Oncology, Vol. 4, No. 1, 2009, pp. 39-55.

Smith, C., C. M. Akujuobi, P. Hamory, and K. Kloesel, "An Approach to Vibration Analysis Using Wavelets in an Application of Aircraft Health Monitoring," Mechanical Systems and Signal Processing, No. 21, 2007, pp. 1255-1272.

Smith, I. F. C., and P. Kripakaran, "Model Based Reasoning for Life-cycle Structural Engineering," Proceedings 1st Int. Symp. on Life-cycle Civil Engineering, Ecole Polytechnique Fédérale de Lausanne (EPFL), Lausanne, Switzerland, 2008, pp. 959-963.

Sofge, D. A., "Structural Health Monitoring Using Neural Network Based Vibrational System Identification," Proceedings of the Australia and New Zealand Conference on Intelligent Information Systems, 1994.

Stockwell, R. G., "Why use the S-Transform," Mathematics Subject Classification, 1991.

Stockwell, R. G., L. Mansinha, and R. P. Lowe, "Localization of the Complex Spectrum: The S Transform," IEEE Transactions on Signal Processing, Vol. 44, No. 4, 1996, pp. 998-1001. 
Stumptner, R., B. Freudenthaler, and J. Küng, "On Similarity in Case-Based Reasoning for Structural Health Monitoring," EUROCAST 2009, LNCS 5717, 2009, pp. 231-238.

Su, Z. Q., L. Ye., and Y. Lu, "Guided Lamb Waves for Identification of Damage in Composite Structures: A Review," Journal of Sound and Vibration, Vol. 295, No. 3, 2006, pp. 753-780.

Sugeno, M., and T. Yasukawa, "A Fuzzy-Logic-Based Approach to Qualitative Modeling," IEEE Transactions on Fuzzy Systems, Vol. 1, No. 1, 1993, pp. 7-31.

Sweetman, B., "SpaceShipOne: Riding a White Knight to Space," Aerospace America, Vol. 42, No. 1, 2004, pp. 45-48.

Suter, D., T. Hamel, and R. Mahony, "Visual Servo Control Using Homography Estimation for the Stabilization of and X4-flyer," Proceedings of the 41st IEEE Conference on Decision and control, Nevada, USA, 2002, pp. 2872-2877.

Tansel, I.N., A. Yenilmez, M. Li, and T. Pamphile, "Index Based Reasoning (IBR) for Total Health Management of UAVs," Presented at the Integrated Systems Health Management (ISHM) Conference, 2006.

Tansel, I. N., M. Li, A. Yapici, A. Yenilmez, "Integrated Systems Health Monitoring for Autonomous Space Access Vehicles and Satellites," 3rd International Conference on Recent Advances in Space Technologies (RAST), June 2007, pp. 187-192.

Trinh, H. P., "Prediction of Engine Performance and Wall Erosion Due to Film Cooling for the 'Fast Track' Ablative Thrust Chamber," NASA, Washington Essays on the History of Rocketry and Astronautics, Vol. 2, 1994, pp. 279-324. 
Tumer, I. Y., and A. Bajwa, "A Survey of Aircraft Engine Health Monitoring Systems," AIAA-99-2528, 35th Joint Propulsion Conference and Exhibit, 1999.

Wang, C. S, F. Wu, and F. K. Chang, "Structural Health Monitoring from FiberReinforced Composites to Steel-reinforced Concrete," Smart Materials and Structures, Vol. 10, No. 3, 2001, pp. 548-552.

Wang, X., and I. N. Tansel, "Modeling the Propagation of Lamb Waves using a Genetic Algorithm and S-transformation," Structural Health Monitoring, Vol.6, No.1, 2007, pp. 23-37.

Wang, Z., "Artificial Intelligence Applications in the Diagnosis of Power Transformer Incipient Faults," PhD Dissertation, Electrical and Computer Engineering, Virginia Tech, 2000.

Watanabe, A., S. M. Olcmen, R. Leland, and K. W. Whitaker, "Soft Computing Applications on SR-30 Turbojet Engine," 1st AIAA Intelligent Systems, Technical Conference, Chicago, IN, September, 2004.

Watts, M. J., and S. P. Worner, "Estimating the Risk of Insect Species Invasion: Kohonen Self-organizing Maps Versus k-means Clustering," Ecological Modeling, Vol. 220, 2009, pp. 821-829.

White, C., B. Whittingham, H.C.H. Li, I. Herszberg, and A. P. Mouritz, "Vibration Based Structural Health Monitoring of Adhesively Bonded Composite Scarf Repairs," 5th Australasian Congress on Applied Mechanics, ACAM2007, December 2007, Brisbane, Australia. 
Worden, K. and J. M. Dulieu-Barton, "An Overview of Intelligent Fault Detection in Systems and Structures," Structural Health Monitoring, 2004, Vol.3, No.1, pp. 85-98.

Wu, J. J., "Liquid-propellant Rocket Engines Health-monitoring-A Survey," Acta Astronautica, Vol. 56, 2005, pp. 347 - 356.

Yang, X. F., C. Zhang, and W. Chen, "A Deflection-Based Bridge Diagnosis Method,"

Mathematical Problems in Engineering, Vol. 2009, 2009, Article ID 284368, 12 pages, $10.1155 / 2009 / 284368$.

Yen, G. G. and P. Meesad, "An Effective Neuro-Fuzzy Paradigm for Machinery Condition Health Monitoring," IEEE Transactions on Systems, Man, and Cybernetics-Part B: Cybernetics, Vol. 31. No. 4, August 2001, pp. 523-536. Zadeh, L. A., "Fuzzy Logic and Approximate Reasoning," Synthese, Vol. 30, 1975, pp. 407-428.

Zhang, B., and Z. Zhu, "Developing a Linear Piezomotor with Nanometer Resolution and High Stiffness," IEEE/ASME Transactions on Mechatronics, Vol.2, No.1, 1997, pp. 22-29.

Zhang, X., R. Xu, C. Kwan, S. Y. Liang, Q. Xie, and I. Haynes, "An Integrated Approach to Bearing Fault Diagnostics and Prognostics," American Control Conference, June 8-10, 2005, Portland, OR, USA, 2005, pp. 2750-2755.

Zou, Y, L. Tong, and G. P. Steven, "Vibration-Based Model-Dependant Damage Identification and Health Monitoring for Composite Structures-A Review," Journal of Sound and Vibration, Vol. 2, 2000, pp. 357-358. 
VITA 


\section{MING LI}

\section{EDUCATION}

Ph.D. Candidate, Mechanical and Materials Engineering Dept., Florida International University, 2005-present.

Ph.D. Program, Electrical and Computer Engineering Dept., Monash University, Australia, 2004-2005.

\section{WORKING EXPERIENCE}

Instructor, Florida International University (Jan. 2007-Dec. 2009)

- Introduction to Computer Aided Design for Mechanical Engineering (Spring 2009, Summer 2009)

- Programming for Mechanical Engineering (Spring 2008)

- Intermediate Computer Aided Design and Computer Aided Engineering (Summer 2008)

- Mechanical Computer Aided Design (Summer 2008)

- Measurement and Instrumentation Lab (Spring 2007, Fall 2007, Fall 2008 )

Teaching Assistant, Florida International University (Aug. 2005-Dec 2009)

- Introduction to Mechatronics and Automatic Control (Spring 2006, Fall 2009)

- System Identification Technology and Signal Processing (Spring 2006, Fall 2009)

- System Dynamics (Summer 2007)

- A/C Design (Summer 2007)

- Smart Machine Design and Development (Spring 2006, Fall 2009)

- Dynamics (Fall 2006)

\section{PUBLICATIONS}

- I. N. Tansel, M. Li, M. Demetgul, K. Bickraj, B. Kaya, B. Ozcelik, "Detecting Chatter and Estimating Wear from the Torque of End Milling Signals by using Index Based Reasoner (IBR)," International Journal of Advanced Manufacturing Technology, 10.1007/s00170-010-2838-5.

- Ibrahim N. Tansel, Ming Li, Gurjiwan Singh, Gurjashan Singh, Srikanth Korla, "Monitoring The Health of Unmanned Aerial Vehicles," UAV2010, Turkey. Accepted.

- Ibrahim N. Tansel, Gurjiwan Singh, Gurjashan Singh, Srikanth Korla, Ming Li, "Unmanned Air Vehicles Health Monitoring Using GONN," UAV2010, Turkey. Accepted.

- M. Li, Gurjiwan Singh, Gurjashan. Singh, A. Yenilmez, M. Demetgul, I. Tansel, "Sweep Sine Wave Based SHM for Short Composite Tubes," The 3rd International Multi-Conference on Engineering and Technological Innovation: IMETI 2010, June 29th - July 2nd, Orlando, Florida, USA. Accepted.

- M. Li, Srikanth Korla, K. Bickraj, X. H. Li, I. N. Tansel, B. Kaya, B. Ozcelik, “On Line Tool Wear Monitoring Using Index Based Reasoning and Self Organizing Map," The 4th International Multi-Conference on Society, Cybernetics and Informatics: WMSCI 2010, June 29th - July 2nd, Orlando, Florida. Accepted. 
- M. Li, M. Demetgul, X. Li, I. Tansel, "Development of Effective Structural Health Monitoring Strategies Using Self Organizing Map and Index Based Reasoning," Proceedings of International Workshop on Structural Health Monitoring, IWSHM2009, Sep. 2009, Stanford, pp. 561-568.

- Ming Li, Mustafa Demetgul, Xiaohua Li, Ibrahim Tansel, "Integrated System Health Management by Using the Index Based Reasoning (IBR) and Self Organizing Map (SOM) Combination," 4th International Conference on Recent Advances in Space Technologies (RAST), June 2009, Turkey, pp. 181-185.

- Ming Li, Mustafa Demetgul, Xiaohua Li, Hillar Lago, Ibrahim Tansel, "Fault Tolerant Fuzzy Logic Control for Four Rotor Helicopter", Florida Conference on Recent Advances in Robotics (FCRAR), May, 2009, Jupiter.

- Li, X., Levy, C., Agarwal, A., Datye, A., Elaadil, L., Keshri, A. K. and Li, M., "Multifunctional Carbon Nanotube Film Composite for Structure Health Monitoring and Damping", The Open Construction and Building Technology Journal, 2009, accepted.

- Xiaohua Li, Cesar Levy, Ming Li, Anup Kumar Keshri, Arvind Agarwal, "A Multifunctional MWCNT Composite: Strain Sensing, Damping and Application to Structural Vibration Control," Proceedings of the ASME 2009 International Mechanical Engineering Congress \& Exposition, IMECE2009, Nov. 2009, Florida.

- Ming Li, Mustafa Demetgul, Ibrahim Tansel, "Single Chip Index Based Reasoner(SCIBR) for Small Satellites," International Workshop On Small Satellites, New Missions and New Technologies, June 2008, Turkey.

- Ibrahim N. Tansel, Ming Li, Ahmet Yapici, "Evaluation of Performance of the Index Based Reasoning (IBR) at a Simulated UAV," The 6th International Workshop Structural Health Monitoring, 2007, pp. 264-268.

- I. N. Tansel, M. Li, A. Yapici, A. Yenilmez, "Integrated Systems Health Monitoring for Autonomous Space Access Vehicles and Satellites," 3rd International Conference on Recent Advances in Space Technologies (RAST), June 2007, pp. 187-192.

- Kimberly Bickraj, Bulent Kaya, Ahmet Yapici, Ming Li, Ibrahim Tansel, Babur Ozcelik, "Inspection of Chatter Damage in End Milling Operations by Using Wavelet Transformations," The Fifth Latin American and Caribbean Conference for Engineering and Technology(LACCEI), Mexico, May, 2007.

- A. Yapici, K. Bickraj, A. Yenilmez, M. Li, I. N. Tansel, S. A. Martin, C. M. Pereira, L. E Roth, "Representation of the Characteristics of Piezoelectric Fiber Composites with Neural Networks," 33rd Annual Review of Progress in Quantitative Nondestructive Evaluation (QNDE 2006), July 2006, Oregan, Portland, pp. 918-926.

- Kimberly Bickraj, Thierry Pamphile, Aylin Yenilmez, Ming Li, Ibrahim N. Tansel, "Fuzzy Logic Based Integrated Controller for Unmanned Aerial Vehicles", Florida Conference on Recent Advances in Robotics and Robot Showcase, May 2006. 